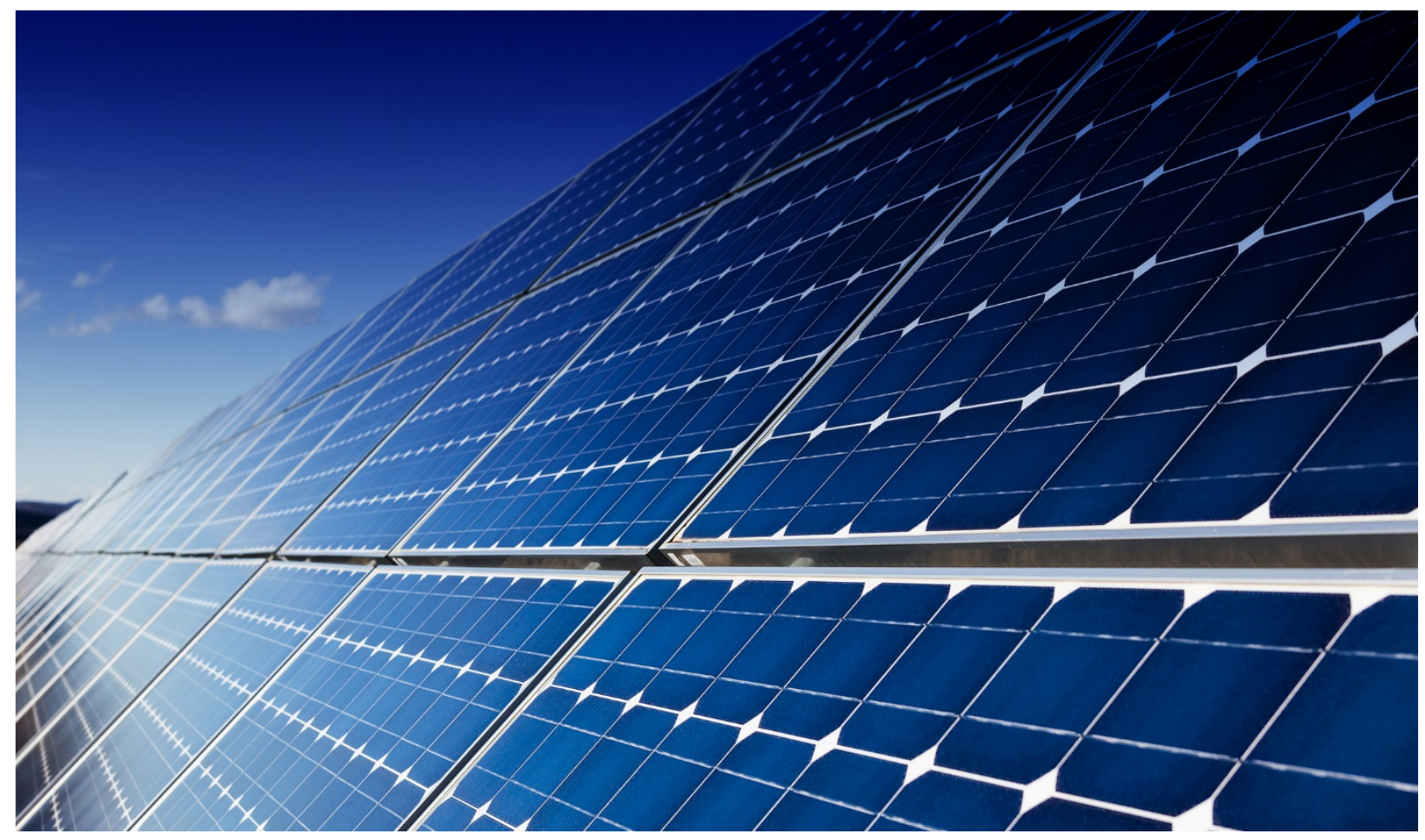

\title{
AN OVERVIEW OF BEHIND-THE-
} METER SOLAR-PLUS-STORAGE PROGRAM DESIGN:

\section{WITH CONSIDERATIONS FOR INDIA}

Owen Zinaman, Thomas Bowen, and Alexandra Aznar U.S. National Renewable Energy Laboratory

June 2020

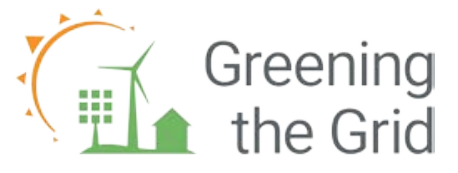

A product of the USAID-NREL partnership

Contract No. IAG-17-2050 


\section{NOTICE}

This work was authored, in part, by the National Renewable Energy Laboratory (NREL), operated by Alliance for Sustainable Energy, LLC, for the U.S. Department of Energy (DOE) under Contract No. DEAC36-08G028308. Funding provided by the United States Agency for International Development (USAID) under Contract No. IAG-17-2050. The views expressed in this report do not necessarily represent the views of the DOE or the U.S. Government, or any agency thereof, including USAID.

This report is available at no cost from the National Renewable Energy Laboratory (NREL) at www.nrel.gov/publications.

U.S. Department of Energy (DOE) reports produced after 1991 and a growing number of pre-1991 documents are available free via www.OSTI.gov.

Cover photo from iStock 471670114.

NREL prints on paper that contains recycled content. 


\section{Acknowledgments}

The authors thank Anurag Mishra from U.S. Agency for International Development (USAID) India Mission for his support and vision, as well as Sarah Lawson from USAID Office of Energy and Infrastructure. We also wish to thank the following individuals for their detailed review comments, insights, and contributions to this report (names listed alphabetically by organization and last name).

Clean Energy Group_-Seth Mullendore

E3 Analytics-Toby Couture

Gujarat Energy Research Management Institute-Akhilesh Magal

Hawai’i Natural Energy Institute-Mark Glick, Marc Matsuura, and Leon Roose

Lawrence Berkeley National Laboratory_-Naïm Darghouth

National Renewable Energy Laboratory (NREL) — Ron Benioff, Joyce McClaren, Michael Coddington, Karlynn Cory, Liz Doris, David Hurlbut, Adarsh Nagarajan, David Narang, Zac

Peterson, Josue Prado, Adam Warren, Andrea Watson and Julianna Williams

Nexus Energy Tech Private Limited - Arvind Karandikar

Regulatory Assistance Project-Ranjit Bharvirkar

Tetra Tech-Sumedh Agarwal, Devina Anand, Ujjwal Bhattacharjee, Rakesh Kumar Goyal, and Ronnie Khanna

University of California-Davis - Sopitsuda Tongsopit

World Bank-Alan David Lee

The authors would also like to acknowledge and thank Britton Marchese, Isabel McCan, and Liz Breazeale of NREL for their editorial support. 


\section{List of Acronyms}

\begin{tabular}{|c|c|}
\hline AHJ & authority having jurisdiction \\
\hline APS & Arizona Public Service \\
\hline BESS & battery energy storage system \\
\hline C\&I & commercial and industrial \\
\hline CEA & Central Electricity Authority \\
\hline CERC & Central Electricity Regulatory Commission \\
\hline $\mathrm{CPP}$ & critical peak pricing \\
\hline CPS & Clean Peak Standards \\
\hline CPUC & California Public Utilities Commission \\
\hline $\mathrm{DBC}$ & declining block charge \\
\hline DER & distributed energy resource \\
\hline DG & distributed generation \\
\hline DISCOM & distribution company \\
\hline DPV & distributed photovoltaic \\
\hline FERC & U.S. Federal Energy Regulatory Commission \\
\hline FOR & Indian Forum of Regulators \\
\hline GWh & gigawatt hours \\
\hline IBC & inclining block charge \\
\hline IEEE & Institute of Electrical and Electronics Engineers \\
\hline IOU & investor-owned utility \\
\hline $\mathrm{kW}$ & kilowatt \\
\hline MW & megawatt \\
\hline NEM & net energy metering \\
\hline NFPA & National Fire Protection Association \\
\hline NREL & National Renewable Energy Laboratory \\
\hline NYISO & New York Independent System Operator \\
\hline OPR & Governor's Office of Planning and Research \\
\hline PSH & pumped storage hydropower \\
\hline RPS & Renewable Portfolio Standards \\
\hline SCE & Southern California Edison \\
\hline SERC & state electricity regulatory commission \\
\hline SGIP & Small Generator Interconnection Procedures \\
\hline TOU & time-of-use \\
\hline UPS & uninterruptible power supply \\
\hline USAID & U.S. Agency for International Development \\
\hline
\end{tabular}




\section{Executive Summary}

Indian consumers have been deploying behind-the-meter generation (predominantly diesel backup, and, more recently, photovoltaic) and storage systems (predominantly lead-acid and other kinds of batteries as uninterrupted power supplies) by the millions for decades (Jaiswal et al. 2017; Seetharam et al. 2013; IFC 2019). These storage systems are used by consumers to address reliability issues within the Indian power system, and their deployment is driven by consumer preference rather than any specific government program or policy. However, the same energy storage systems could provide additional services to the consumer and distribution companies if properly regulated and designed from the outset to be grid interactive. Grid-connected distributed solar PV (DPV), or rooftop solar, has also seen wide deployment in India and features prominently in the Government of India's plans for a transition to clean, reliable, and affordable energy for all. At the same time, many utilities and state governments, as well as the central government in India are currently funding-constrained for both operational and future capital expenditures in the power sector, and some perceive customer-sited resources as exacerbating existing financial challenges.

In that context, behind-the-meter energy storage systems paired with distributed photovoltaic (DPV)with the capability to act as both generation and load-represent a potentially unique and disruptive power sector technology capable of providing a range of important services to customers, utilities, and the broader power system in India. Globally, jurisdictions with high penetration of DPV have seen faster uptake of behind-the-meter energy storage systems, such as in California and Hawaii (GTM and Energy Storage Association 2019). India, with more than $4 \mathrm{GW}$ of installed rooftop solar, is primed for the uptake of behind-the-meter energy storage, as consumer economics become more attractive with the fastfalling cost of energy storage systems. A proper framework to coordinate the deployment and operation of these distributed systems can balance stakeholder benefits from their presence on the grid. Without appropriate regulations or technical requirements, however, these systems could potentially 1) cause safety concerns for the utility; 2) exacerbate utility revenue losses; or 3) limit the ability for stakeholders to achieve certain policy goals. This report aims to offer a comprehensive, evidence-based approach to designing customer programs based on experience in the United States that can help regulators, utilities, and policymakers in India manage the range of challenges and opportunities that increased behind-themeter energy storage deployment will bring to the power system, in particular when these systems are paired with DPV.

This report has been prepared by the National Renewable Energy Laboratory (NREL) with support from the U.S. Agency for International Development (USAID) for discussion purposes with a broad range of stakeholders. These include Indian regulatory agencies (such as the Forum of Regulators, the Central Electricity Regulatory Commission, and various State Electricity Regulatory Commissions), policy makers, utilities, and developers to inform a broader dialogue around the future direction of Indian states' approach to regulating and facilitating DPV-plus-storage systems. Importantly, this report is intended to offer key regulatory considerations for facilitating DPV-plus-storage programs for retail customers. As the role of regulators is often to convene and balance the interests of a broad range of stakeholders, including policymakers, utilities and customers, this report focuses on their role in the development of behind-the-meter DPV-plus-storage programs. Throughout the report, relevant cases from U.S. states are provided as examples of how novel regulatory issues related to behind-the-meter energy storage systems paired with distributed photovoltaic are being addressed in practice. ${ }^{1}$

\footnotetext{
${ }^{1}$ The United States and India share similar governance structures in the electricity sector. As well, the majority of states in both nations also feature (or at one time featured) net energy metering (NEM) as a core distributed generation policy. Because of these similarities, this report predominantly focuses on cases from U.S. states to illustrate potentially transferrable lessons to the Indian context.
} 
Indian policymakers may be interested in utilizing DPV-plus-storage to enable rooftop solar goals, improve the financial health of distribution companies (DISCOMs), expand customer choice, and/or reach electrification targets, among other drivers. Indian DISCOMs may be interested in utilizing DPV-plusstorage resources to reduce their operational and capital expenditures, manage network congestion, reduce peak demand, or even drive new revenue via the creation of customer-centric DPV-plus-storage offerings. Indian consumers, on the other hand, may be more focused on improving the quality or reliability of their electricity, reducing their electricity bills, or taking advantage of new DISCOM program offerings. In any case, through the design of DPV-plus-storage programs, policymakers and regulators have an opportunity to create new market opportunities for Indian consumers, DISCOMs and project developers, and at the same time make significant strides toward additional goals for the power sector.

This report offers an overarching framework for Indian policymakers, regulators and other stakeholders to consider as they begin to contemplate the desired role of DPV-plus-storage in the Indian power system, as well as the potential suite of policies, programs, and regulations that might enable its deployment. At a high level, designing a framework that aligns DPV-plus-storage deployment with larger policy objectives requires thoughtful deliberation across a range of technical and economic issues. While this report attempts to segment many of these issues into distinct topics to enhance reader understanding, in reality, DPV-plus-storage regulatory issues are all closely integrated and rapidly evolving, and design decisions on a single aspect often have broader implications. With that in mind, this report outlines a series of steps that can be employed by regulators and other stakeholders to approach DPV-plus-storage program design (see Figure ES- 1).

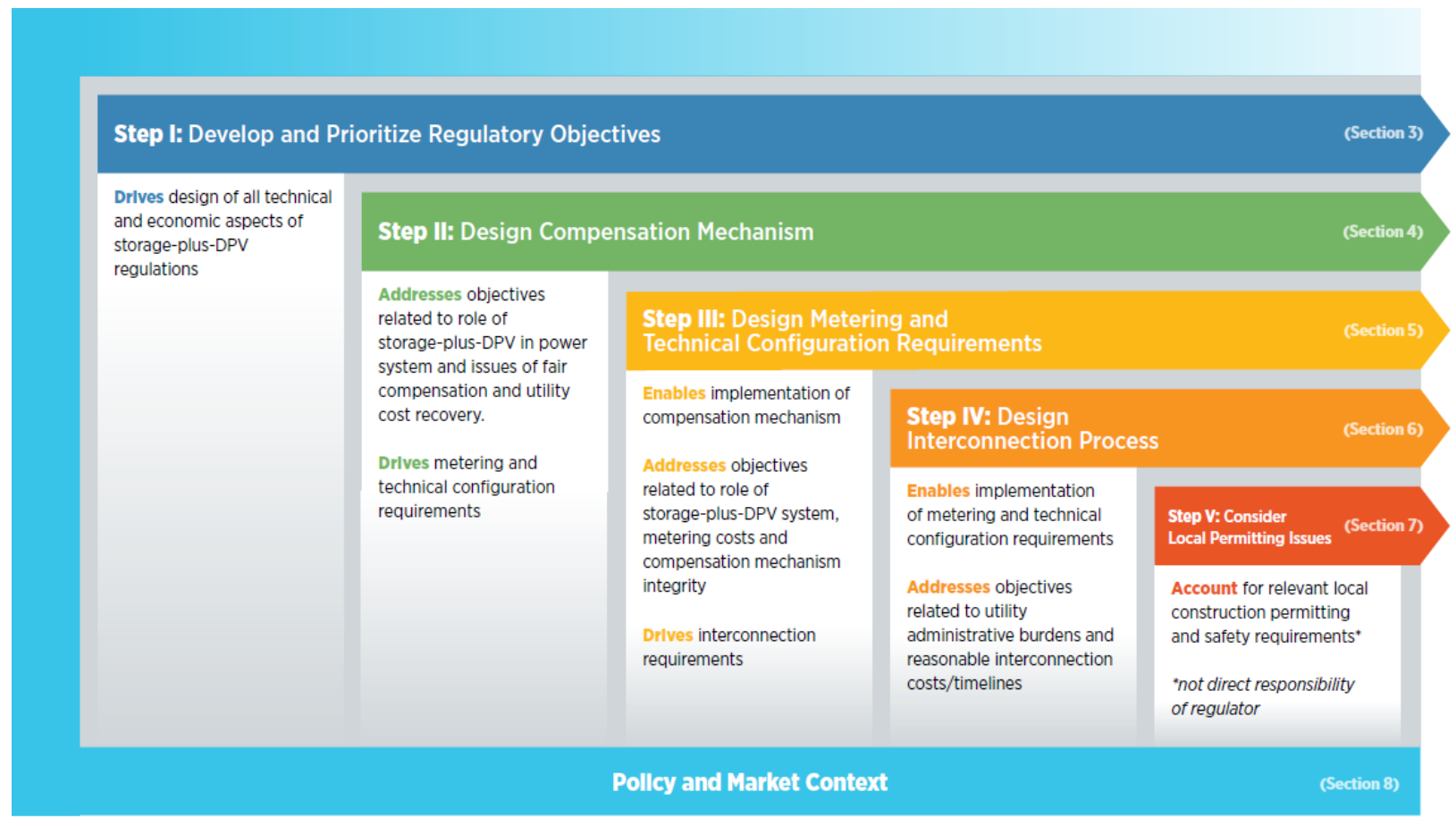

Figure ES-1. Approaching program design for DPV-plus-storage systems

Source: Original [Illustration by Christopher Schwing, NREL]

Policy and Market Context-The policy and market context into which DPV-plus-storage systems are being deployed will invariably influence the objectives regulators will want to pursue. Regulators can take into consideration the presence of stated policy goals, as well as local power system conditions, when considering their objectives and plans of action. 
Step 1: Develop and Prioritize Regulatory Objectives-As a first step, developing clear regulatory objectives - which may be influenced by, aligned with, or directly derived from stated public policy goals - can help guide individual decisions on designing regulations. Batteries located behind the meter of a retail customer are technically capable of providing myriad services across all voltage levels of the power system. Regulators can consider a range of important objectives, including the desired role and behavior of DPV-plus-storage systems in the power system to provide services, appropriate levels of financial and administrative burdens for customers and utilities, utility cost recovery and crosssubsidization issues, and operational transparency and safety, among others. Often times regulators will pursue objectives which may potentially conflict with one another, or will want to place more emphasis on some objectives over others. Prioritizing identified objectives can help regulators balance a diverse and sometimes conflicting set of objectives that can guide the overall program design process.

Step 2: Design Compensation Mechanism-Next, regulators can consider the appropriate compensation mechanism offerings for DPV-plus-storage systems that attempt to address and balance objectives related to fair customer compensation and utility cost recovery, as well as the desired role of DPV-plus-storage in the power system. This report provides a broad overview of metering and billing arrangements, retail rate design options, and sell rate design options that may be relevant for DPV-plus-storage customers.

Importantly, decisions surrounding compensation mechanisms influence rules related to metering and technical configuration requirements for DPV-plus-storage systems.

Step 3: Design Metering and Technical Configuration Requirements—Rules governing metering and technical configuration requirements for DPV-plus-storage can be designed to ensure the desired compensation mechanism can be implemented, and that the desired role of DPV-plus-storage systems will be realized in the power system. Technical configuration requirements affect the ability of DPV-plusstorage to interact with the grid by potentially restricting certain flows of energy, and ultimately influence the role that these systems can play in the power system. Metering requirements enable the implementation of compensation mechanisms and can be used to increase the visibility of DPV-plusstorage systems to the utility; however, they also have important cost implications for regulators to consider.

Step 4: Design Interconnection Process-Regulators play an important role in developing interconnection processes for DPV-plus-storage systems, in some cases directly driving these efforts, and in other cases overseeing the utility's efforts. Designing a process for DPV-plus-storage systems to interconnect to the power system in a safe and orderly manner requires the development of: (1) rules governing the procedures that utilities employ to manage the interconnection application process; (2) rules governing how DPV-plus-storage equipment is expected to perform and interact with the power system; and (3) rules governing how interconnection applications are screened and evaluated, with a specific focus on how DPV-plus-storage systems will impact operations on the distribution circuit where the system will be constructed.

Step 5: Consider Local Permitting Issues - While not directly under their purview, regulators must nevertheless account for the presence of local construction permitting processes, including compliance with relevant building, electrical, and fire safety codes. These codes are critical to ensuring that DPVplus-storage systems perform safely and as expected, regardless of environmental conditions, and do not suffer performance degradation due to improper installation, operation, or transportation While many local permitting authorities have developed familiarity with DPV systems, behind-the-meter storage is a new and evolving topic with which local governments may not have significant experience. Regulators can help build local capacity with storage systems by sharing information resources and contributing to the development of model permitting procedures. 


\section{Key Considerations for Indian Stakeholders}

This report has identified nine key considerations for regulators, policymakers, and other key stakeholders when designing programs for behind-the-meter DPV-plus-storage systems, based on evidence from similar programs in the United States.

\section{High-level Considerations}

Well-timed regulatory decisions can enable Indian DISCOMS to seize the opportunities presented by customer deployment of advanced battery technologies. Indian consumers have been installing behind-the-meter lead-acid batteries for several decades to address reliability issues in the Indian power system. These systems are typically designed for backup purposes, and broadly speaking lack the technical characteristics to become grid-interactive and benefit DISCOMs. As lithium-ion battery costs continue to decline, there may soon be a significant opportunity to enroll customer-sited lithium-ion batteries to reduce DISCOM operating costs and improve power system reliability. Regulations which address advanced inverter capability requirements, communication protocols, and customer compensation can be designed proactively to seize this opportunity and improve the financial health of DISCOMs.

Determining the desired role of DPV-plus-storage is an important first step. Compared to grid-tied DPV systems, DPV-plus-storage has a significantly broader set of capabilities to offer customers and the power system, and policymakers and regulators can help to guide how these resources are ultimately utilized. The design of the DPV-plus-storage program will be determined by stakeholders' power system objectives and their relative priority. Some may wish to see DPV-plus-storage systems deployed to primarily or explicitly serve a customer's own energy demand, and/or for individual customer backup purposes during grid outages. Some Indian utilities, however, are already considering how to deploy DPV-plus-storage systems to provide grid services to the power system, as are many private developers. Currently storage systems in India are encouraged or required to operate in an entirely passive manner, e.g. to meet load during outages, and there has been limited efforts by State Electricity Regulatory Commissions (SERCs) to-date to explicitly encourage the deployment of these systems or to use these systems to achieve specific objectives, especially for more grid-interactive roles. This leaves room for SERCs to clearly articulate their objectives and desired use cases for storage systems more clearly.

Customizing rules and requirements based on the characteristics of the DPV-plus-storage system is a key strategy for SERCs to promote fairness. Creating distinct sets of compensation mechanisms, metering and technical configuration requirements, and interconnection processes - which may be based on system size, the intended use of the system (e.g., an exporting versus non-exporting storage system), and other aspects - can help ensure regulatory requirements are appropriate for various segments of the DPV-plus-storage market in India.

\section{DPV-plus-Storage Compensation Considerations}

Current state net energy metering (NEM) policies do not incentivize storage deployment. Although two states in India have moved away from conventional NEM (Gujarat and Maharashtra), most Indian states currently feature a NEM framework for DPV that effectively activates the grid as a "financial battery" for customers. While NEM is an effective incentive for grid-tied DPV systems, access to such a free financial battery is likely more favorable to customers than the capital expenditure associated with a new behind-the-meter battery system. As Indian states begin transitioning to net billing schemes (as discussed in the Forum of Regulator's Model Regulation for Grid Interactive Distributed Renewable Energy Sources document), the value of storage to customers to reduce their bills may increase, as storage can be used to reduce DPV grid injections and increase DPV self-consumption.

Tariff design is an important tool to align the interests of DPV-plus-storage customers with the broader power system in India. In India, most customers are subject to flat (time-invariant) volumetric 
rates, with some larger customers subject to Time-of-Day rates and demand charges. Flat volumetric rates provide little opportunity for customers to reduce their bills through storage systems. Alternative retail tariffs designed specifically for DPV-plus-storage customers - featuring structures such as time-variant rates (e.g., Time-of-Day) or demand charges - could incentivize the coupling of storage and solar, as well as help align customer behavior with system needs. For instance, the USAID PACE-D 2.0 Renewable Energy team submitted storage-friendly tariff proposals to regulators in Gujarat and Jharkhand, suggesting the creation of time-variant demand charges and the partial relaxation of minimum billing demand requirements. Considerations for placing customers on more advanced tariff structures must be considered in the context of the customers' ability to meaningfully alter their consumption in response to price signals from the power system, as many smaller customers may be unable to change their demand to take advantage of new tariffs.

Indian regulators can enable business model innovation for DPV-plus-storage systems. Regulators can play a key role in allowing alternative investment and ownership models for DPV-plus-storage systems. For instance, both third-party ownership schemes and utility-led "Bring-Your-Own-Device" incentive programs are already beginning to play a role in storage deployment in the United States, Australia, and elsewhere. These programs have allowed distribution utilities to utilize storage assets when they would otherwise sit idle to reduce the cost of service and increase system reliability. In the Indian context, the prospect of DISCOMs utilizing customer-sited, customer-financed storage resources in exchange for a modest fixed monthly bill credit may be particularly appealing for cash-constrained Indian DISCOMS. In the future, behind-the-meter storage systems might be aggregated by third parties to provide valuable services to DISCOMs and/or the bulk power system. Indian regulators can play a key role in enabling the participation of aggregators and in some cases, defining how they are allowed to operate.

\section{Technical Regulation and Safety Considerations}

Metering and technical configuration requirements for DPV may need to be revisited to incorporate more grid-interactive applications of DPV-plus-storage. In India, most existing regulations do not explicitly address the interaction of storage with the power system, nor do they explicitly state which power flows in DPV-plus-storage systems should be measured or prohibited. In jurisdictions that want to take advantage of the various services that batteries can provide, such requirements will likely need to be updated to allow for discharging power to the grid. However, making changes to technical regulations may have cost implications for customers or DISCOMs (e.g., the cost of a new smart meter), and SERCs can carefully weigh these costs against the benefits they yield. Differentiating metering and technical configuration requirements based on customer class, voltage interconnection level, or system size may help minimize undue cost burdens for customers, as well as administrative costs for utilities that must review and approve these systems.

\section{Existing DPV regulations in India can be used as foundation for designing DPV-plus-storage}

interconnection rules. In India, the large majority of existing rules related to distributed energy resources are focused on the interconnection of DPV systems. Updating these rules to more effectively leverage the potential grid support benefits of behind-the-meter batteries will likely be an incremental effort which builds upon existing interconnection rules developed by SERCs and guidance from the Central Electricity Authority (CEA). Given existing distributed solar quality issues in India, procedures to verify battery inverter settings may be of particular interest for SERCs to explore. Additional quality control procedures may also be merited to ensure communication between the battery and system operators is secure and reliable, should behind-the-meter batteries ever be utilized as a resource by system operators to provide grid services. Importantly, many SERCs look to the central government in India for support on the development of technical interconnection rules. In that context, the CEA can consider updating the 2013 Technical Standards for Connectivity of the Distributed Generation Resources (CEA, 2013) regulations to include appropriate grid requirements for behind-the-meter storage and DPV-plus-storage systems. 
Concurrently, SERCs can consider directing DISCOMs to begin developing appropriate technical requirements for behind-the-meter storage.

\section{Both central and state governments in India can play an essential role in developing and facilitating} familiarity with new safety codes and inspection procedures for behind-the-meter storage. The introduction of behind-the-meter DPV-plus-storage systems will likely require the development of additional wiring and fire safety codes (and associated inspection procedures) focused on battery safety that would be enforced by DISCOMs and state Chief Electrical Inspectorate to Government (CEIG) offices in India. Central and state governments can consider promulgating best practice manuals for enforcing battery wiring and fire safety codes, and/or holding workshops to boost familiarity with inspectors. Regulators and policymakers can also encourage, and help fund, pilot projects to inform which regulations will be most needed and how those regulations might impact storage system deployment and operation.

With these considerations, India is poised to capture the myriad benefits of modern distributed energy resources, including DPV-plus-storage, in a systematic manner while balancing multiple stakeholder interests. Through a thoughtful design process for supporting policies, regulations, and utility programs, Indian state and central government leaders have a significant opportunity to create new opportunities for resilience, clean energy deployment, and economic growth, while making substantial progress toward additional established power sector transformation goals. 


\section{Table of Contents}

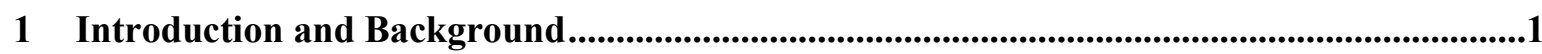

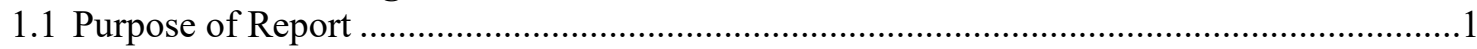

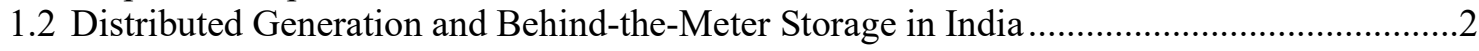

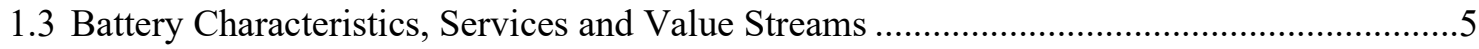

1.3.1 Background: Increasing Prominence of Battery Storage ..................................... 5

1.3.2 Background: Battery Services and Value Streams............................................ 6

1.3.3 Relevant Market Conditions and Battery Applications for Indian Context ........... 8

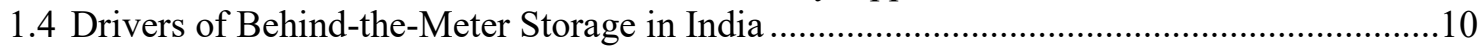

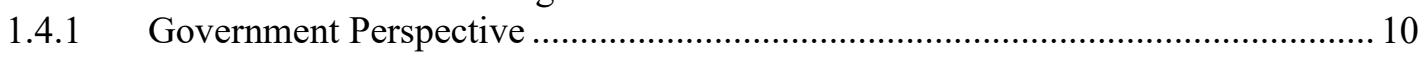

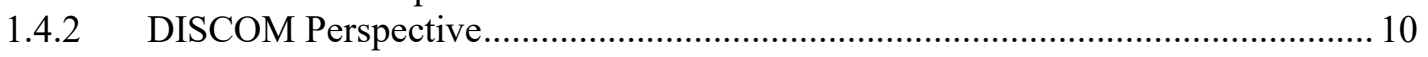

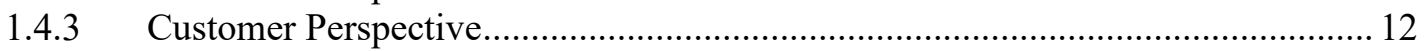

1.5 Storage Market Context: Global and United States ..........................................................13

2 Approaching DPV-Plus-Storage Program Design........................................................................16

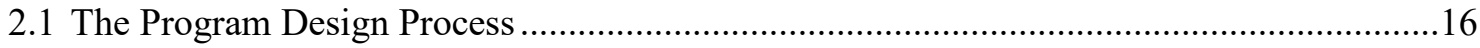

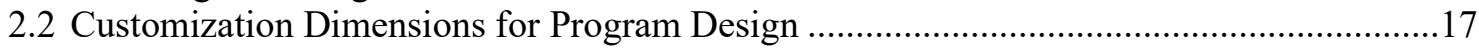

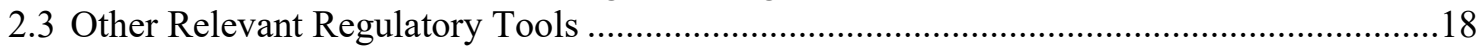

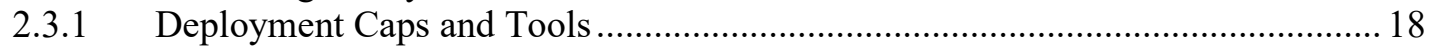

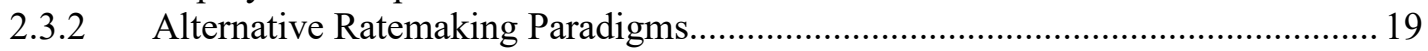

3 Step I: Develop and Prioritize Regulatory Objectives .................................................................20

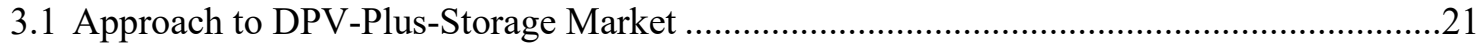

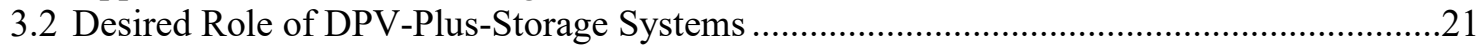

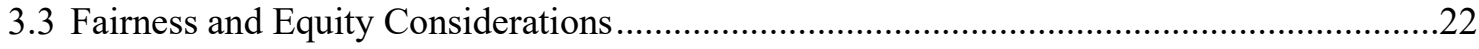

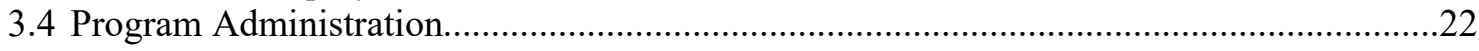

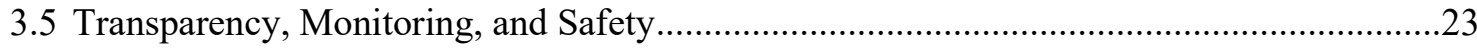

4 Step II: Design Compensation Mechanism .....................................................................................25

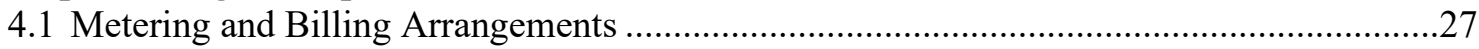

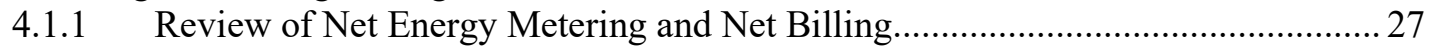

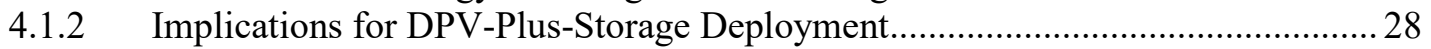

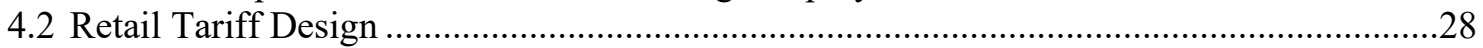

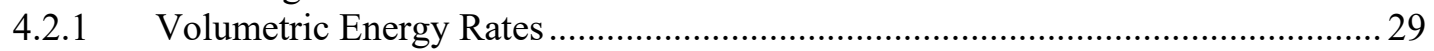

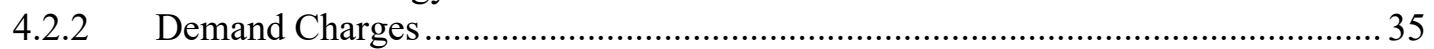

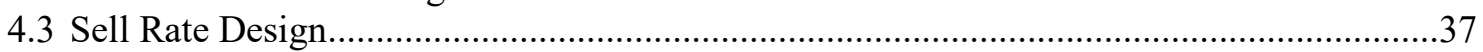

5 Step III: Design Metering and Technical Configuration Requirements ..............................38

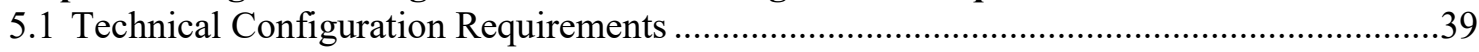

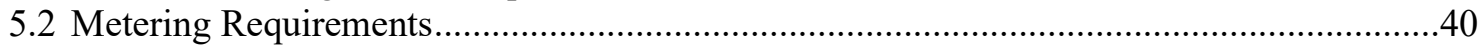

5.3 Preserving Compensation Mechanism Integrity ........................................................... 41

5.3.1 Using Estimation Methods for DPV-Plus-Storage Export Crediting.................... 41

5.3.2 Ensuring Accurate Metering Through Design Configuration Limitations ............. 42

5.3.3 Require Adequate Metering to Track All Quantities Relevant for Preserving

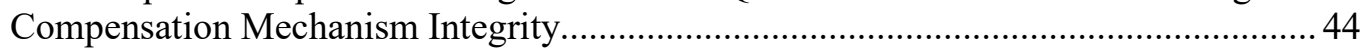

6 Step IV: Design Interconnection Rules....................................................................................46

6.1 Rules Governing the Interconnection Application Procedures and Management ................48

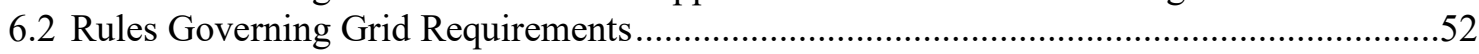

6.2.1 Interconnection Requirements and Underlying Relevant Standards.....................52

6.2.2 Equipment Requirements and Underlying Relevant Standards ............................53

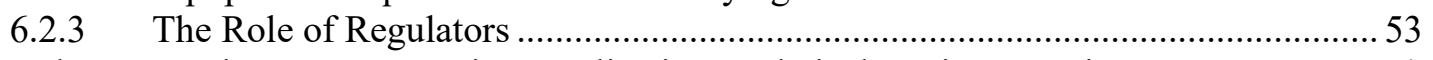

6.3 Rules Governing Interconnection Application Technical Review Practices .......................54

6.3.1 Review of Distributed Energy Resource Technical Screening Processes...............54

6.3.2 Distinctions for Screening DPV-Plus-Storage Systems........................................ 56 


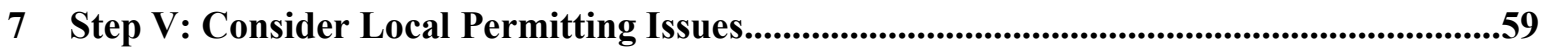

8 Policy and Market Context for DPV-Plus-Storage .............................................................62

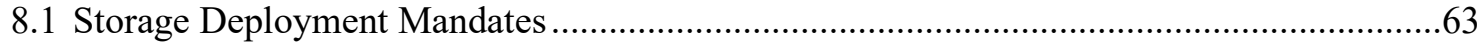

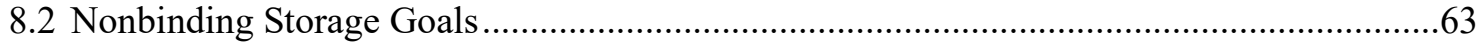

8.3 Renewable Portfolio Standards and Clean Peak Standards ..............................................64

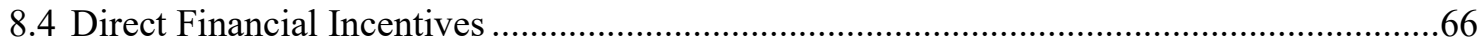

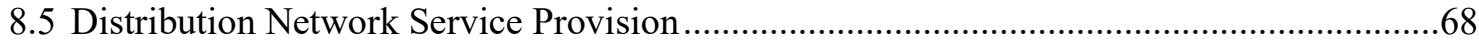

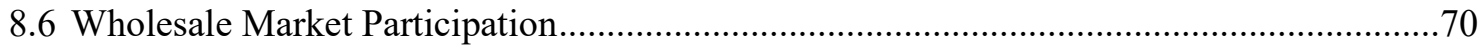

9 Concluding Remarks: Considerations for DPV-plus-Storage Program Design...................72

Appendix A-Energy Storage Technology Ecosystem ..........................................................74

Appendix B-Tradeoffs of Various Battery Technologies.....................................................76

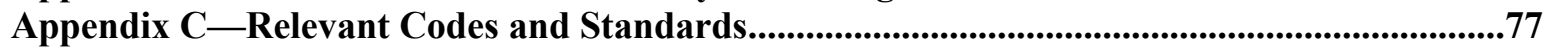

Appendix D-DPV-Plus-Storage Technical Configurations .........................................................80

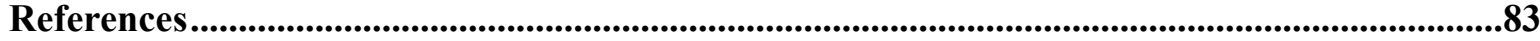




\section{List of Figures}

Figure ES- 1. Approaching program design for DPV-plus-storage systems

Figure 1. Average global lithium-ion battery pack price declines, including year-over-year percentage cost declines.

Figure 2: Summary of potential battery energy storage services and required response times and durations

Figure 3. Estimated annual distributed grid and off-grid PV-plus-storage power capacity and expected vendor revenue by region, world markets: 2017-2026..

Figure 4. Cumulative U.S. deployment of energy storage by capacity and number of systems for front-ofthe-meter and behind-the-meter systems from Q1 2013 to Q1 2019

Figure 5. U.S. state deployment of energy storage by capacity of behind-the-meter and front-of-the-meter

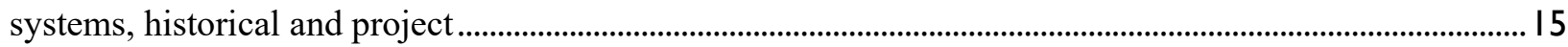

Figure 6. Approaching program design for DPV-plus-storage systems............................................................ 17

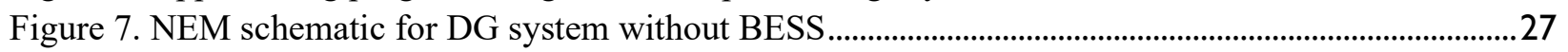

Figure 8. Net billing schematic for DG system without BESS .......................................................................28

Figure 9. Illustrative diagram of inclining block rate (left) and declining block rate (right)..........................33

Figure 10. Energy origins, pathways, and destinations for behind-the-meter solar-plus-DPV customers ..39

Figure 11. Typical DER interconnection and permitting process........................................................................48

Figure 12. Overview of technical screening process for an interconnection application ...............................56

Figure 1. Storage technology types and applications organized by storage medium ........................................75

Figure D- 1. AC-coupled DPV-plus-storage system with backup enabled ........................................................8I

Figure D- 2. DC-coupled DPV-plus-storage system with multiport hybrid inverter with backup enabled $8 \mathrm{I}$

\section{List of Tables}

Table 1. Definitions of Key Battery Technical Characteristics …............................................................

Table 2. Customization Dimensions for Regulatory Design ............................................................ 18

Table 3. Roles and Responsibilities for Interconnection Application Procedures and Management Rules 51

Table 4. Qualified Energy Storage System Charging Windows by Renewable Energy Source................ 66

Table 5. California Small Generator Incentive Program Budget and Incentive Levels.............................67

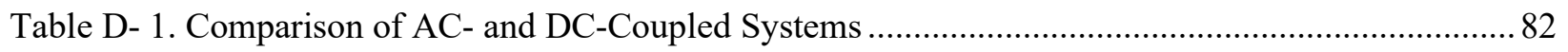




\section{Introduction and Background}

\subsection{Purpose of Report}

Behind-the-meter energy storage systems paired with distributed photovoltaic (DPV) — with the capability to act as both generation and load - represent a unique and disruptive power sector technology. ${ }^{2}$ Such assets represent a powerful new opportunity to more efficiently, cost-effectively and reliably operate power systems, and could prove fundamental to achieving an array of stated policy objectives in India. This report offers a comprehensive, evidence-based approach to designing customer programs based on experience in the United States - it is intended to serve as a foundational knowledge base from which regulators, utilities, and policymakers in India can manage the range of challenges and opportunities that increased behind-the-meter energy storage deployment will bring to the Indian power system, in particular when these systems are paired with DPV. Although multiple parties will play important roles in the development of programs for DPV-plus-storage, this report focuses on the role of regulators, as they are often tasked with convening and balancing the interests of a broader range of stakeholders.

This report has been prepared by the National Renewable Energy Laboratory (NREL) with support from the U.S. Agency for International Development (USAID) for discussion purposes with a broad range of stakeholders. These include Indian regulatory agencies (such as the Forum of Regulators, the Central Electricity Regulatory Commission, and various State Electricity Regulatory Commissions), policy makers, utilities, and developers to inform a broader dialogue around the future direction of Indian states' approach to regulating and facilitating DPV-plus-storage systems. Importantly, this report is intended to offer key regulatory considerations for facilitating DPV-plus-storage programs for retail customers. As the role of regulators is often to convene and balance the interests of a broad range of stakeholders, including policymakers, utilities and customers, this report focuses on their role in the development of behind-the-meter DPV-plus-storage programs. Notably, broader strategies to reconceptualize regulatory frameworks - such as revenue decoupling ${ }^{3}$ or performance-based regulation ${ }^{4}$ - may also be useful for the reliable integration of DPV-plus-storage systems. These strategies are discussed briefly in Section 2.3.2, but are not the focus of this report. Throughout the report, relevant cases from U.S. states are provided as examples of how novel regulatory issues related to behind-the-meter energy storage systems paired with distributed photovoltaic are being addressed in practice. ${ }^{5}$

Section 1 discusses how batteries, in particular lithium-ion batteries, fit into the larger ecosystem of energy storage technologies, outlines the various system services that batteries can provide, discusses the concept of value stacking, and provides context on the U.S. energy storage market, as well as expectations of global growth in energy storage deployment.

Section 2 outlines the program design process, discusses how policy objectives can influence downstream decisions such as compensation mechanism design, and highlights important tools available to decisionmakers to customize regulations to fit specific market and power system contexts.

Section 3 discusses various potential regulatory objectives for DPV-plus-storage programs and describes a general framework for approaching DPV-plus-storage regulatory issues.

Section 4 reviews various considerations for the design of DPV-plus-storage compensation mechanisms.

\footnotetext{
${ }^{2}$ This report defines behind-the-meter storage as a stationary electrochemical storage system connected directly to a retail consumer of grid electricity, as opposed to systems installed on the grid side. The term behind-the-meter derives from the idea that user-side systems are 'behind' the user's utility service meter from the perspective of the utility.

${ }^{3}$ See Revenue Regulation and Decoupling: A Guide to Theory and Application prepared by the Regulatory Assistance Project.

${ }^{4}$ See Next Generation Performance-based Regulation prepared by the Regulatory Assistance Project and NREL.

${ }^{5}$ The United States and India share similar governance structures in the electricity sector. As well, the majority of states in both nations also feature (or at one time featured) net energy metering (NEM) as a core distributed generation policy. Because of these similarities, this report predominantly focuses on cases from U.S. states to illustrate potentially transferrable lessons to the Indian context.
} 
Section 5 explores a range of technical considerations, strategies, and approaches for developing metering and technical configuration requirements for DPV-plus-storage systems.

Section 6 discusses the development of interconnection processes and technical requirements for DPV-plusstorage.

Section 7 briefly describes local permitting issues surrounding DPV-plus-storage systems.

Section 8 reviews a range of policy instruments and approaches to encourage DPV-plus-storage deployment.

Section 9 offers a summary of key regulatory considerations for DPV-plus-storage systems.

\subsection{Distributed Generation and Behind-the-Meter Storage in India}

Indian consumers have been deploying behind-the-meter generation (predominantly diesel backup, and, more recently, photovoltaic) and storage systems (predominantly lead-acid and other kinds of batteries as uninterrupted power supplies) systems by the millions for decades (Jaiswal et al. 2017; Seetharam et al. 2013; IFC 2019). These systems are used by consumers to address reliability issues within the Indian power system, and their deployment is driven by individual action. Their operation is in an entirely passive mode with respect to the power system (i.e., delivering power to the customer more or less automatically during service interruptions on the distribution system). When grid electricity service is resumed, these behind-the-meter systems either switch off (e.g., diesel generators) or begin charging (e.g., lead-acid batteries). Indian distribution companies (DISCOMs) do not typically have operational awareness of these systems, nor do they typically have any control over them. Achieving DISCOM access to these existing battery systems would likely be expensive and logistically challenging, as neither the requisite advanced inverters nor the communication infrastructure are currently in place to control them.

Meanwhile, DPV features prominently in the Government of India's plans for a transition to clean, reliable, and affordable energy for all. In December 2015, the government approved a Grid-Connected Rooftop and Small Solar Power Plants Programme for installation of 4,200 megawatts (MW) of DPV in the country by the 2019-20 fiscal period (MNRE 2014). As of September 2019, cumulative rooftop solar deployment in India was 5,252 MW, with over $70 \%$ of deployment occurring in the commercial and industrial (C\&I) sectors. In February 2019, the government approved Phase II of the program to achieve a cumulative capacity of 40,000 MW of rooftop solar by $2022^{6}$, including 4,000 MW specifically in the residential sector (MNRE 2019). Each Indian state has been assigned a specific schedule of annual deployment targets for rooftop solar (MNRE 2015), and there is an increased focus by many states on achieving these targets.

One determinant of grid users' interest in DPV is the rate and structure of grid electricity tariffs. Tariff design also influences the financial impact that DPV have on utilities. Indian retail tariffs are predominantly time-invariant for small consumer classes, with larger consumers also offered or required to be on time-of-day rates. These tariffs are often inclining block in nature, with increasing levels of consumption charged higher rates (Patel et al. forthcoming). Thus large consumers (who are predominantly commercial and industrial customers, but also include high-use, wealthy residential customers) tend to pay more, while small consumers (who are predominantly poor and mostly residential and agricultural customers) tend to pay less on a per-unit energy basis. These inclining block tariffs have created a strong cross-subsidy scheme in which utilities must recover surplus revenue from larger consumers in excess of the cost of providing them electricity, to supply smaller consumers with electricity at rates below the actual cost of providing them electricity (Patel et al. forthcoming; Josey et al. 2018).

Often, the tariffs for C\&I consumers are already higher than the levelized cost of DPV (and possibly even some storage, depending on the specific DISCOM and consumer segment). As a result, some C\&I consumers in India may have a strong economic incentive to reduce grid consumption through self-generation if they have available space and capital to invest in DER systems (Josey et al. 2018; Jaiswal et al. 2017). Consequently, DISCOMs have

\footnotetext{
${ }^{6}$ The national goal of 40,000 MW of rooftop solar is part of a broader goal to achieve 100,000 MW of solar photovoltaic
} deployment in India by 2022. 
started experiencing reduced C\&I demand, as these consumers begin installing DER systems (Jaiswal et al. 2017). As of September 2019, 74\% of installed rooftop solar capacity in India has been deployed by C\&I customers (Bridge to India 2019). This trend further reduces DISCOM's intake of cross-subsidy revenues (i.e., the funding yielded from larger customers intended to subsidize smaller customers). If C\&I tariffs continue to rise to recover additional cross-subsidy revenue - for example, in the form of higher demand-based charges - this could create an incentive for $\mathrm{C} \& \mathrm{I}$ consumers to begin investing in storage, further encouraging the load defection of C\&I consumers and exacerbating existing utility cost recovery issues. In some states, regulators and DISCOMs have moved to limit the revenue implications of DPV adoption by reducing the incentives for installing such systems. For example, in Maharashtra regulators plan to impose "grid support charges" for rooftop solar (Parikh 2020); in Gujarat, regulators have placed size limits on net-metered rooftop solar systems based on the size of the customer's 'sanctioned load' or 'contract demand' (GERC 2016); in Karnataka regulators have sought to end Net Energy Metering altogether in favor of gross metering (KERC 2019).

Despite the cross-subsidy system, Indian utilities are routinely unable to recover their fixed operating costs, and their respective state governments (that often own the utilities) must often provide the utility a subvention from the state budget (RBI 2017; Buckley 2015; GOI 2019a). As these financial losses accumulate over multiple years, the central government has historically intermittently stepped in with some sort of a bailout policy (e.g., through the Ujjwal DISCOM Assurance Yojana ${ }^{7}$ program), as these losses threaten to infect the balance sheets of the public sector banks (who are the primary lenders in India), which can affect overall economic growth (Buckley 2015).

Of late, there has been reduced electricity demand growth in the C\&I sectors (where cross-subsidy funding originates) relative to residential and agricultural consumption (where cross-subsidy is deployed and electrification programs are focused) (BNEF 2019; GOI 2019a). Many Indian utilities are unable to fully recover their costs and have already stopped paying generators in full and on time (GOI 2018). The banking sector that lends to the power sector, meanwhile, is under stress due to the many nonperforming assets to whom they have loaned and may have a reduced appetite to further lend in the power sector (Buckley 2015). The central government's balance sheet, as well as those of most states, is also under significant stress (RBI 2017). In summary, utilities, state governments, and the central government in India are currently funding-constrained for both operational and future capital expenditures, though the exact situation and circumstances vary widely among individual settings.

This broader economic situation has implications for customer-sited resources, which are often perceived to exacerbate existing financial challenges. In reality, many customer-sited resources (including behind-the-meter storage and, in some cases, rooftop solar) present an opportunity to assist financially ailing DISCOMs by reducing costs associated with operating the distribution system (Kuldeep et al. 2019). Seizing that opportunity may require changes to both prevailing attitudes toward the role and impact of customer-sited resources and approaches to distributed energy resource regulation and policy more broadly.

\footnotetext{
${ }^{7}$ See: https://www.uday.gov.in/home.php
} 


\section{Behind-the-Meter Storage in the Indian Context}

Behind-the-meter storage and generation systems have been ubiquitous in India for several decades to address grid reliability issues for customers. These behind-the-meter systems typically consist of a diesel or, more recently, solar PV generator, combined with some form of short-term energy storage device, primarily lead-acid batteries. They are almost always operated in a grid-passive mode, automatically meeting consumer demand when the distribution system experiences an outage and switching off and/or charging when grid electricity is restored. The storage in these systems typically operates as a UPS, acting quickly to ensure the continuous availability of power in the event of service interruption. Importantly, these systems have historically had little to no interaction with the larger power system in India.

This report refers to behind-the-meter storage as any system technically capable of: (1) charging from either the grid, a co-located power source (e.g., solar PV), or both; and (2) discharging to meet customer demand and/or into the distribution network. In addition to providing backup power or power quality services, these advanced systems have the capability to help retail customers reduce their electricity bills and maximize their use of on-site generation. Furthermore, these systems are typically technically capable of grid interactivity (i.e., providing a wide range of power, capacity, and ancillary services to the broader power system and responding to time-variant and demandbased retail electricity tariff structures to contribute to the cost-effective operation of the power system), as opposed to previous/existing storage systems in India, which have historically been designed with the sole purpose of customer reliability. These behind-the-meter systems can be comprised of a large range of storage technologies, but in leading markets around the world, are currently dominated by lithium-ion.

Although DPV-plus-storage systems may not currently be economically attractive relative to DPV systems for most Indian consumers ${ }^{8}$, developing regulations and requirements - such as those outlined in this report before lithium-ion systems begin to deploy at-scale may be a highly valuable undertaking. Although India has seen significant deployment of behind-the-meter storage systems, these systems are not equipped to provide any services beyond backup power. Existing behind-the-meter storage systems in India, which have been neither expected nor required to meaningfully interact with the power system, tend to lack advanced inverters. Without advanced inverter functionality - not to mention utility communication infrastructure - such systems may lack the sophistication to provide many grid services. Furthermore, most existing behind-the-meter storage systems in India are lead-acid batteries. Relative to lithium-ion technologies, lead-acid batteries have shorter cycle-life, meaning that they are not as well suited for many applications that require frequent charging from and discharging to the grid (Fan et al. 2020). However, moving forward, with proactively designed regulations in place, Indian DISCOMS can be ready to seize the opportunities presented by large-scale deployment of customer-sited lithium-ion assets to reduce their operating costs and improve power system reliability.

\footnotetext{
${ }^{8}$ See 'Customer Economic Analysis of Behind-the-Meter DPV-plus-Storage' text box in Section 1.4.3.
} 


\subsection{Battery Characteristics, Services and Value Streams}

\subsubsection{Background: Increasing Prominence of Battery Storage}

Storage deployment has historically been dominated in terms of capacity by utility-scale pumped hydropower storage (PSH) systems connected to medium- or high-voltage transmission networks ${ }^{9}$; however, new installations around the world increasingly use electrochemical battery storage technologies, both in large-scale utility-side (i.e., front-of-the-meter) and smaller user-side (i.e., behind-the-meter) applications. Battery energy storage systems (BESS) can comprise many different chemistry types, including lithium-ion, lead-acid, nickel-based, sodium-based, and redox flow. Each of these chemistries differs in their technical characteristics, and each have their own relative advantages and disadvantages that make them suitable for different grid system applications. Table 1 defines a variety of technical characteristics of batteries, whereas Appendix B discusses key tradeoffs among various battery technologies.

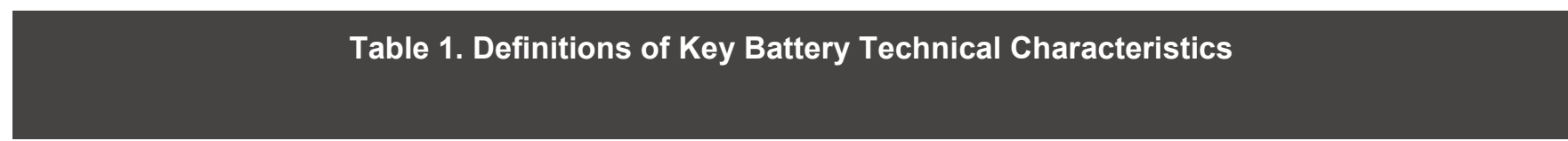

Rated Power Capacity

\section{Energy Capacity}

Energy/Power Density
Total possible instantaneous discharge capability (in kilowatts [kW] or megawatts [MW]) of the BESS, or the maximum rate of discharge that the BESS can achieve, starting from a fully charged state.

Maximum amount of stored energy (in kilowatt-hours [kWh] or megawatt-hours [MWh]) a battery can hold (capacity may also be represented as 'Usable Energy Capacity' or 'Operating Energy Capacity' that reflects the highest percentage of the total energy capacity recommended to preserve battery performance).

Specific Energy/Power Measure of the energy or power capacity of a battery relative to its weight $(\mathrm{kW} / \mathrm{g}, \mathrm{kWh} / \mathrm{g})$.

Storage Duration

Amount of time storage can discharge at its rated power capacity before depleting its energy capacity. $\begin{array}{ll}\text { Cycle Life/Lifetime } & \begin{array}{l}\text { Amount of time or cycles a battery storage system can provide regular charging and discharging } \\ \text { before failure or significant degradation. }\end{array}\end{array}$

Round-trip

Efficiency

Self-discharge
Ratio of the energy charged to the battery to the energy discharged from the battery.

Reduction of stored energy of the battery (\% of charge/time) through internal chemical reactions, rather than through discharging to perform work.

Source: Bowen et al. (2019)

For additional information on battery characteristics, see MIT Electric Vehicle Team (2008).

Lithium-ion batteries have, in recent years, become the most commonly newly installed battery technology for power sector applications, deploying in both front-of-the-meter and behind-the-meter applications. The increasing prominence of lithium-ion batteries is due to steep price declines caused by technological innovation and expanded manufacturing capacity. Manufacturing capacity is expected to continue to grow to serve growing demand from the transportation and power sectors and has been forecasted to reach a global annual manufacturing capacity of over 1,000 gigawatt hours (GWh) by 2025, up from $119 \mathrm{GWh}$ in 2017 , an increase of over $740 \%$. Furthermore, from 2010-2018, average lithium-ion battery pack prices declined from $\$ 1,160 / \mathrm{kWh}$ to $\$ 176 / \mathrm{kWh},{ }^{10}$ a reduction of over $80 \%$, with significant continued price declines predicted for the coming decade (see Curry 2017; BNEF 2019). Figure 2 shows global average lithium-ion battery price declines since 2010 .

\footnotetext{
${ }^{9}$ For a concise overview of the energy technology ecosystem, see Appendix A.

${ }^{10}$ In this report, unless otherwise noted, $\$$ shall refer to USD.
} 


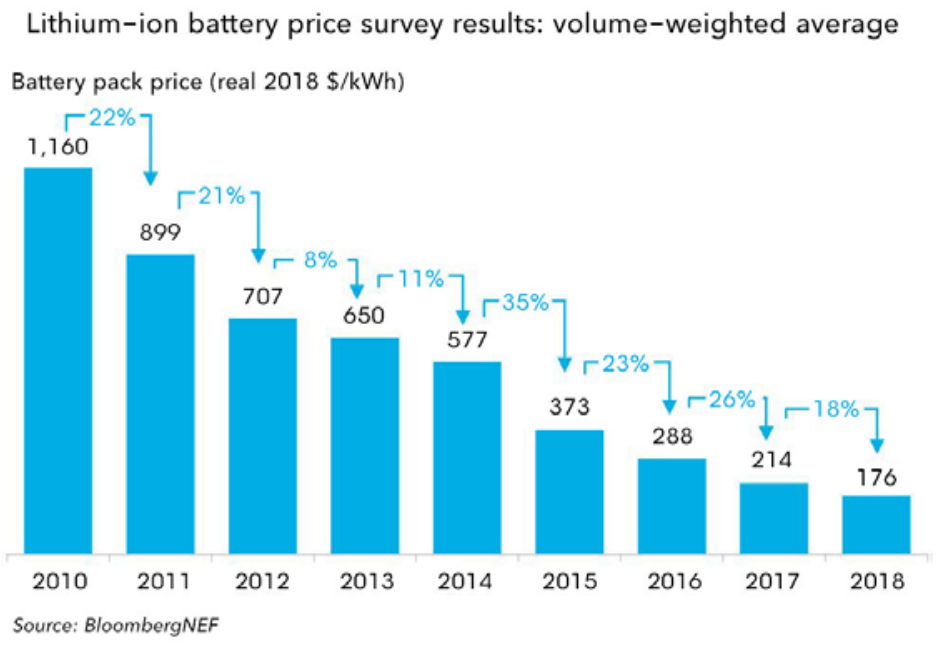

\title{
Figure 1. Average global lithium-ion battery pack price declines, including year-over-year percentage cost declines
}

\author{
Source: BNEF 2019
}

For behind-the-meter applications in particular, lithium-ion chemistries have been the most dominant battery technology due to a number of considerations. First, battery cells and packs are modular, and behind-the-meter projects tend to benefit from the same global price reductions due to manufacturing economies of scale as larger utility-scale systems. Second, many of the alternative storage options are simply not practical for behind-themeter applications, due to aspects such as size (i.e., flow batteries) or safety considerations (i.e., sodium-based batteries). Third, lithium-ion batteries offer a higher relative energy density and durability, as well as better charge times, relative to other battery chemistries that may otherwise be suitable for behind the-meter applications.

At the moment, India is predominantly dependent on imports for lithium-ion batteries for energy storage applications (Afonso 2020). Import duties for already-manufactured lithium-ion cells currently sit at 10\%, and may increase over time as the domestic manufacturing ramps up. The Indian central government has recently began taking steps to support the localization of lithium-ion battery supply chains through its existing Make in India program and the creation of the National Mission on Transformative Mobility and Battery Storage. As of the time of this writing, several policies have been approved by the Ministry of Finance, but approval is still pending from the Cabinet. Policies pending approval include a significant cash incentive program for battery manufacturers with targeted manufacturing dates to begin in 2022, accelerated depreciation, and a relaxation of import duties on key input materials (e.g., lithium, cobalt) (GOI 2019b; Sharma 2020). Taken in sum, domestically produced lithium-ion batteries, in addition to the continued imports of lithium-ion and other advanced batteries, may become increasingly accessible to Indian consumers in the near-future as the government takes steps to increase local manufacturing.

\subsubsection{Background: Battery Services and Value Streams}

Batteries are a unique power system asset in that they can provide a highly diverse range of system services, spanning ultra-short-term timescales (i.e., subseconds to seconds) to medium-term timescales (i.e., hours to days). While other conventional resources can also provide these services, battery storage systems tend to do so more quickly and accurately, improving their potential contribution to power system reliability and flexibility.

Figure 2 depicts the various system services that batteries can provide, as well as a color-coded indication of whether these services are commonly valued and monetizable in power systems around the world. The left side of each bar depicts the required response time of the battery for each service, whereas the right side of the bar depicts the maximum duration of time for which the storage resource typically must provide the service. Notably, the figure also depicts services these battery systems can provide based on where the battery is interconnected to the power system (i.e., behind-the-meter, distribution system, or transmission system). Batteries generally do not provide system services to segments of the power system with lower voltage levels than they are connected to but can provide services to higher voltage levels. For instance, a battery located on the distribution system can provide services to the distribution and transmission network, but not to individual customers for demand charge 
reductions. Batteries located behind-the-meter of a retail customer, on the other hand, are technically capable of providing services across all voltage levels. It should be noted that Figure 2 only illustrates what services batteries would be technically capable of supplying; however, the effective provision of these services by behind-the-meter battery systems in particular will require both enabling communication technology and mechanisms to adequately compensate battery system owners or operators for services provided (see text box: Market and Policy Barriers to Value Stacking in Section 8 for more information on nontechnical barriers to DPV-plus-storage systems providing system services).

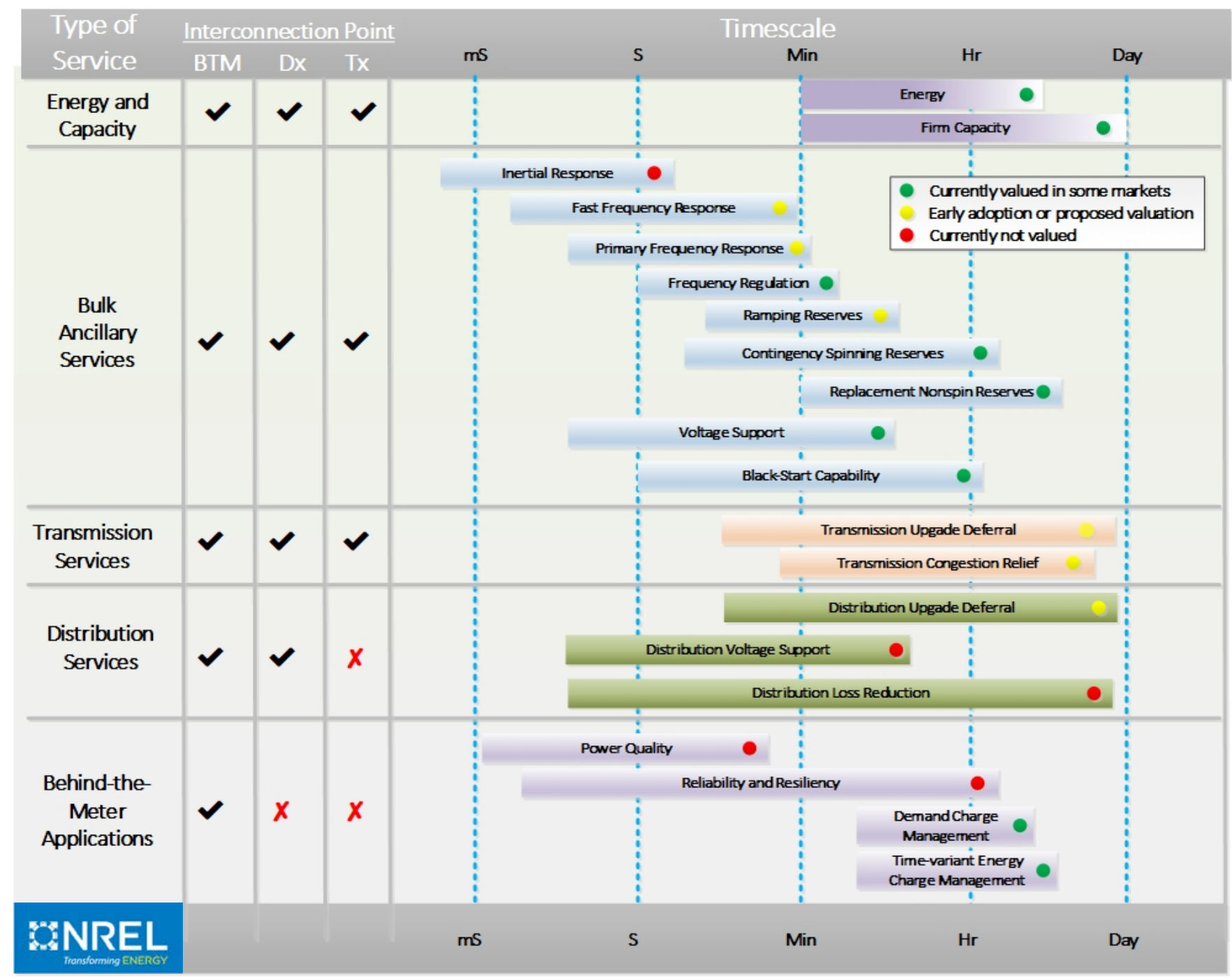

Figure 2: Summary of potential battery energy storage services and required response times and durations

Source: Original [IIlustration by Vahan Gevorgian, Paul Denholm \& Owen Zinaman, NREL]

Importantly, battery storage systems can have enhanced value to the power system and system owners by providing one and sometimes multiple distinct system services. Battery system operators can increase the utilization of their systems by providing distinct system services valued in the market for distinct durations at different times of day. This multi-use approach is known as "value stacking" and is increasingly being explored and implemented for grid-interconnected batteries, particularly in markets that offer more than one way to be paid for those system benefits. Value stacking can be an essential strategy for battery storage system owners, as monetizing multiple system services can improve battery economics.

Although battery systems in general may be technically capable of providing multiple services, there are several important limitations to consider as regulators seek to facilitate the ability of behind-the-meter DPV-plus-storage customers to pursue value stacking activities. To begin, not all services that batteries are technically capable of 
providing are necessarily (or adequately) compensated, depending on the specific power system context, regulatory framework and location and interconnection voltage of the battery. Second, given that many of the services that batteries can provide require very different operating characteristics (e.g., duration and depth of discharge, response time), optimizing a battery system's technical performance for any single application, or even groups of applications may require excluding that battery system from effectively providing other groups of services. Even in the event that a range of services could theoretically be monetized, in practice it may be difficult for a battery to operate in an optimized manner to maximize its value to the system owner as well as the broader power system as the provision of one particular service may preclude the provision of others. For instance, if a battery commits to providing essential reliability services for a period of time (e.g., spinning reserves for a 3-hour period), in practice it may be impeded from other profit-seeking activities during that period (e.g., provision of energy following an unexpected wholesale market price spike). Furthermore, for smaller behind-the-meter systems, control algorithms are not yet widely available to allow customers to program or optimize batteries to take advantage of multiple value streams. Finally, as batteries provide an increased number of services, it is possible they will cycle more frequently than if optimizing their operations to provide a narrower set of services. Depending on the particular battery chemistry, this increased cycling could lead to an accelerated degradation of various battery performance metrics - thus, there may be additional operational costs associated with some value stacking activities that must be weighed against the benefits.

\subsubsection{Relevant Market Conditions and Battery Applications for Indian Context}

There are many potential use cases and value streams for storage in India. However, the applicability of these use cases can vary widely with the local set of market conditions for each Indian state or DISCOM service territory. Key market conditions that may steer a state or DISCOM toward emphasizing particular value streams and use cases for behind-the-meter storage are listed below.

Regular reliability issues and/or unmanaged peak demand - Some Indian states and cities regularly struggle to meet demand due to low reserve margins, transmission congestion issues, quickly growing peak demand, among other issues ${ }^{11}$. Under these circumstances, storage may be an attractive option for consideration. To begin, retail electricity customers may be more likely to deploy behind-the-meter storage when they are otherwise faced with regular outages. Utilities may wish to enroll these demand-side storage resources to manage peak demand, and/or build utility-scale storage facilities to help time-shift demand and provide a range of important ancillary services.

Regular power quality issues - India currently suffers from persistent power quality issues that inhibit the functioning and competitiveness of the Indian economy as well as negatively impact consumer experience (Prayas Energy Group 2019; FOR 2018). Promoting distribution-connected or customer-sited storage could be an effective use case for addressing localized power quality issues on distribution networks caused by regular grid operation or higher localized penetrations of rooftop solar (India Smart Grid Forum 2019). Regulators overseeing service territories with power quality issues may consider allowing DISCOMs to rate base storage assets, and/or re-designing tariffs to incentivize retail customers to deploy storage. Furthermore, specific customers may already be more likely to employ behind-the-meter storage to ensure both reliable power as well as high-quality power, especially in electronics manufacturing or in data centers where power quality issues can significantly impair endproducts or services.

Distribution network congestion - In some cases, particularly in areas experiencing demand growth, peak demand within a particular segment of the distribution system may exceed the physical limitations of the equipment needed to provide power, such as transformers or lines. During these peak demand periods, the DISCOM may be unable to fully satisfy demand or may need to dispatch generators out of merit order to fully meet demand, operating the power system in an economically sub-optimal way which leads to higher power prices. During off-peak hours, however, portions of the power system may be over-sized relative to demand, leading to lower utilization rates. In order to delay or offset the need to upgrade the distribution system, utilities

\footnotetext{
${ }^{11}$ For instance, in a recent Electricity Supply Monitoring Initiative monthly report, which collected outage and interruption data for 133 locations across India, 53\% of locations experienced at least one outage of more than 15 hours in length. $31 \%$ of locations experienced more than 30 service interruptions that were greater than 15 minutes in length, whereas $27 \%$ of locations experienced average daily outages of 30 minutes or more during evening hours (Prayas Energy Group 2019).
} 
might choose to invest in storage systems, or incentivize the adoption of customer-sited storage systems, within a congested area. These systems can charge during periods of lower demand and meet demand during peak hours locally, helping DISCOMs supply customers in all hours, or ensure that generators continue to be dispatched in merit order, even during peak periods. Also, unlike traditional transmission or distribution investments, mobile storage systems could be relocated to new areas when no longer needed in the original location, increasing their overall value to the DISCOM (Bowen et al. 2019).

Support for DPV integration on high penetration feeders - The presence of DPV systems on distribution feeders can, under some circumstances, exacerbate distribution-level issues, such as DPV systems on the ends of long feeders leading to higher voltages 'upstream'. Although these issues typically do not manifest until very high local DPV penetrations, and can often be resolved using improved interconnection standards and inverter requirements (see Sections 6.2 and 6.3), storage systems can also be used to manage the exports from DPV systems in order to minimize their negative impact on the grid. For instance, storage systems could help reduce the presence of coincident exports on a feeder with high levels of DPV penetration, such that not all systems would export power at the same time and cause reverse power flow. Storage could also be directly interconnected to the distribution network to address periods of oversupply of DPV.

Aggressive DPV deployment targets - Related to the previous market indicator, the integration of larger amounts of DPV deployment, driven by aggressive DPV targets, may become a challenge to DISCOMs over time. The deployment of distribution-connected storage devices strategically located within the sub-network of particular feeders or transformers may help to mitigate these issues (India Smart Grid Forum 2019). Furthermore, encouraging customers to pair DPV systems with behind-the-meter storage devices (e.g., through compensation mechanism design - see Chapter 4) may have the two-fold benefit of reducing the impact of individual DPV systems while simultaneously contributing to the mitigation of power quality or congestion issues on the broader distribution network.

Financially unhealthy DISCOMs - While some DISCOMs undoubtedly represent success stories for financial sustainability and turnaround, many still struggle with their financial and operational sustainability. For instance, the total outstanding payments of DISCOMs to generation companies as of November 2019 was Rs. 729.4 billion (Ministry of Power 2019) ${ }^{12}$. Although potentially counterintuitive, DPV and DPV-plus-storage systems can potentially help financially ailing DISCOMs by reducing their costs associated with operating the distribution system. For example, through interconnection requirements, tariff design, and in some cases new programmatic offerings, DISCOMs can enroll DPV-plus-storage systems to manage peak demand, defer/avoid network upgrades, and/or to meet otherwise unserved demand. As well, if DISCOMs must disburse a large portion of their income subsidizing certain customer classes (as is commonplace throughout most of India), there may be circumstances in which it is actually more cost effective for DISCOMs to install a DPV or DPV-plus-storage system at the customer premise and encourage self-generation, rather than heavily-subsidized consumption from the grid.

High electricity prices - One possible value stream for DPV and DPV-plus-storage adoption is customer bill savings through the reduction of consumption from the grid. Bill savings can come both from the reduction of volumetric charges (INR/kWh) as well as from changes in demand patterns, such as reducing consumption during peak periods under time-of-use rates (see section 4.2.1.2) or reducing a customer's maximum rate of consumption during certain periods under demand charges (see section 4.2.2). Although bill savings will likely be a key driver of storage deployment in many cases, for customers subject to higher electricity prices or more complex tariffs, bill savings will play a more central role in the adoption of DPV and DPV-plus-storage systems as the potential for savings is increased. Well-designed retail tariffs can also ensure that benefits accrue to utilities as customer behavior becomes aligned with system needs (see section 4.2)

Increasing penetrations of utility-scale wind and solar energy - As penetrations of variable resources increase on the power system, power system flexibility becomes an increasingly important issue. While it is by no means

\footnotetext{
${ }^{12}$ Data collected by Government of India Payment Ratification And Analysis in Power procurement for bringing Transparency in Invoicing of generators (PRAAPTI) Initiative representing 93 participating generation companies and 65 participating DISCOMs.
} 
the only available source of power system flexibility, ${ }^{13}$ Indian states experiencing or expecting higher penetrations of utility-scale wind and solar energy may be interested in storage to manage the additional variability and uncertainty that these variable renewable energy (VRE) resources introduce into the power system. If deployed strategically, storage assets - both customer-sited behind-the-meter and distribution-interconnected systems may be a very useful resource in India for balancing supply and demand, managing network congestions issues, and/or providing frequency services, among others.

\subsection{Drivers of Behind-the-Meter Storage in India}

\subsubsection{Government Perspective}

The following are potential drivers of behind-the-meter storage deployment in Indian states for government agencies.

Enabling Rooftop Solar Goals - India has significant ambitions for rooftop solar, $40 \mathrm{GW}$ by 2022, which could potentially be stymied by technical limitations at the distribution level on certain circuits. In order to meet targets, regulators may be interested in increasing the deployment of storage with DPV systems, which could help mitigate certain DISCOM technical concerns while allowing for the continued deployment of DPV systems. Regulators could achieve this by providing incentives for pairing storage with DPV, particularly in constrained or congested areas.

Improving DISCOM Financial Situations - Policymakers and regulators in India are concerned with the financial health of DISCOMs within their jurisdiction (GOI 2019a). While storage and DPV can reduce the volumetric sale of electricity, and therefore DISCOM revenues, these systems can also help reduce operating costs by meeting peak demand, reducing congestion, deferring or avoiding network investments, and through the provision of other system services. Furthermore, compared to other grid investments, deployment of behind-themeter storage and DPV mobilizes investment in the power system from outside the retail rate base, which may be particularly important for cash-constrained DISCOMs. Regulators in India could consider incentivizing the deployment of such systems among heavily-subsidized customer classes - such as residential and agricultural customers - which has the potential to reduce the subsidy burden of DISCOMs while meeting rooftop solar deployment targets and renewable purchase obligations and providing DISCOMs with valuable grid resources.

Expanding Customer Choice - Policymakers and regulators in India who are interested in expanding customer choice can enable customers to meet portions (or all) of their demand through DPV or DPV-plus-storage.

Furthermore, regulators with sensitive industries in their jurisdiction, such as semiconductor manufacturing or large data centers, or industries with their own clean energy ambitions can ensure that these customers have ready access to DPV and storage to support uninterrupted operations. .

Reaching Electrification Targets - As a part of the Rajiv Gandhi Grameen Vidyutikaran Yojana, regulators in India may be particularly interested in DPV and storage as a means of achieving rural electrification ambitions. Particularly for remote rural areas with little to no existing infrastructure, it may be more cost effective to develop remote microgrids using DPV, storage and other distributed energy resources to provide these customers with low- or no-cost power, either until connection with the main power system can be established or on an indefinite basis.

\subsubsection{DISCOM Perspective}

The following are potential drivers of behind-the-meter storage deployment in Indian states for DISCOMs.

Reducing Operational and Capital Expenditures - Customer-sited DER - including behind-the-meter DPVplus-storage - can be utilized to reduce utility and state/central government operational and capital expenditures. Under the appropriate set of policy and regulatory conditions, DER could conceivably be used to strengthen the financial position of utilities and state/central governments in India, while helping to improve quality of service and reduce bills for individual customers. This could principally occur through enrolling customer-sited DER to

\footnotetext{
${ }^{13}$ For a more in-depth discussion of available strategies for power system operators to increase system flexibility, see (IEA
} 2018). 
help mitigate grid outages, integrate renewable energy, and defer or avoid investments in peaking capacity and the transmission and distribution systems. Activities to seize this opportunity could principally feature changes to retail tariff design (see Section 4.2), associated enabling metering infrastructure (see Sections 5.2 and 5.3), and could be further buttressed by robust grid requirements and processes (see Sections 6.1-6.3) and DISCOM programmatic offerings (see Section 8.5). Such activities may also require political and regulatory leadership, which coordinates efforts with ongoing central government initiatives to deploy advanced metering infrastructure and implement real-time energy market pricing (CERC 2018). Customer-sited DER can also reduce the costs for utilities associated with providing power to heavily subsidized customer classes. For instance, in India, not only are agricultural customers heavily subsidized, they are also often at the end of long radial feeders. DPV-plusstorage systems for these customers could potentially reduce the cost of delivering electricity to such customers while also providing better overall power quality and availability.

Managing Congestion - Congestion on the distribution system can lead both to unmet demand (blackouts) as well as inefficient dispatching of generators out of merit order, increasing operating costs. DISCOMs can use storage and DPV-plus-storage to mitigate congestion on the distribution system by charging during periods of low demand when distribution infrastructure is under-utilized and meeting demand locally during peak periods. This can be particularly important in areas where demand is growing or in areas with aging infrastructure. Furthermore, as storage systems could be designed to be moved to new locations, these assets could help financially stressed DISCOMs defer or spread out upgrades to the distribution system, saving them considerable costs. As different feeders may experience congestion during different time periods, reducing congestion may require direct utility control or precise signals to customers (such as under Critical Peak Pricing schemes - see Section 4.2.1.5). DISCOMs could offer customers on congested feeders increased incentives or rebates to install storage in exchange for periodic control over these systems. Such direct control may require both regulatory guidance as well as sophisticated monitoring and communication requirements (see Sections 6.2 and 6.3 for more information on processes for ensuring adequate behind-the-meter storage capabilities).

Reducing Peak Demand - India is currently experiencing rapid growth in peak demand, with national peak demand growing by over 5\% between the 2018-19 and 2019-20 fiscal periods, and some regions and states growing significantly more (CEA 2018; 2019a). While likely reflective of increasing economic activity, this growth in peak demand can also make it more difficult and more expensive for DISCOMs to avoid outages. DISCOMs can reduce peak demand through the coordinated dispatch of behind-the-meter storage and DPV-plusstorage systems, meeting demand locally through stored energy and reducing the need to either turn on more expensive generating units or purchase power from regional exchange markets. This could be especially cost effective if the storage systems would be used to offset subsidized consumption during peak periods. Peak demand reductions could be initiated either indirectly through cost-reflective tariff design (see Chapter 4) or directly through DISCOM control of assets. For the latter, sufficient communication infrastructure (see Sections 6.2 and 6.3) would be necessary as well as utility-led programs to enlist customers to participate in demand reduction efforts (see Section 8.5 for examples of such utility programs).

Monetizing New Value Streams - In addition to reducing costs and addressing technical issues, distributed storage also offers DISCOMs opportunities to expand into new business models. DISCOMs may be uniquely positioned to act as an intermediary between Indian customers, who may not have a sophisticated understanding of the complexities surrounding storage, and developers who may not have sufficient relationships with customers to understand their needs or reach them. These new business models would likely require explicit regulatory approval by relevant State Electricity Regulatory Commissions to ensure appropriate consumer protections are in place and established regulatory principles for fairness and competition are upheld. Although there are a wide range of business models available, given growing demand and electrification goals in India, these might include:

- providing backup power 'as-a-service', in which DISCOMs lease storage systems to customers for uninterrupted, continuous power, even during grid outages in exchange for regular monthly payments;

- developing microgrids under a similar fixed payment approach, in which utilities coordinate with developers to supply remote, unelectrified regions with regular power separate from the main power system

- relying on non-wire alternatives to offset the need for distribution system upgrades, using storage, energy efficiency and demand response to reduce peak demand on a circuit and increase infrastructure utilization. 


\subsubsection{Customer Perspective}

The following are potential drivers of behind-the-meter storage deployment in Indian states for customers.

Reliability and/or Power Quality Issues - To date, the primary driver of behind-the-meter storage deployment in India has been reliability (and to some extent power quality) issues in the Indian power system. Retail customers regularly utilize storage systems to serve as an uninterruptable power supply, often coupled with DPV or diesel generators, to help meet otherwise unmet demand for electricity. In the future, customers may use battery energy storage systems that charge from the grid or a DPV system as a primary energy source to manage reliability events. In states which have higher reserve margins and less frequent reliability issues (e.g., Gujarat), storage systems may be less attractive to retail customers.

Electricity Bill Savings - Another driver of behind-the-meter storage deployment is the potential for bill savings. Indian customers who have more complex retail tariff structures, including many C\&I customers, may have potential to reduce their bills by strategically shifting demand to take advantage of time-of-day energy charges or demand-based charges. Issues related to retail tariff design and behind-the-meter storage are discussed in Section 4.2 .

\section{Customer Economic Analysis of Behind-the-Meter DPV-plus-Storage}

Customers decide to invest in distributed energy resources such as DPV or DPV-plus-Storage because it provides either indirectly monetizable benefits, such as backup power during outages, or directly monetizable benefits, such as bill reductions, in excess of the long-term costs of purchasing (or leasing) the system. One way to quantify the economic factors that influence customer behavior is through a customer economic analysis. A customer economic analysis can consider the optimum sizing and dispatch of a DER asset to minimize customer bills, given information about that customer's tariff; assumptions about relevant financing considerations (e.g., access to capital, terms of loans, and discount rates, among others), and; installation costs for the system in question. The analysis, in addition to system sizes and optimal battery dispatch patterns, can produce financial performance metrics such as customer billing savings and cash flow estimates, net present value (NPV) of the investment, and the customer payback period.

Researchers from NREL and LBNL performed a customer economics analysis for the state of Gujarat to determine under which conditions DPV and DPV-plus-Storage systems might become economically viable in the state. The analysis found that under present cost assumptions and retail tariffs, customers - on the basis of bill savings alone the economics of standalone DPV systems were significantly stronger than DPV-plus-Storage systems. For instance, it was determined that for larger industrial customers, installed costs of a storage system would have to decrease to $\sim 50 \%$ of their current costs in order for it to be more economic to invest in an DPV-plus-Storage relative to a DPV system. Changes to the demand charge structure of retail tariffs (see Section 4.2.2) for industrial customers, however, reduced the required storage cost declines to a $25 \%$ reduction in order for customers to choose DPV-plus-storage systems. To place these price declines in perspective, from 2010 to 2018 the compound annual decline rate for lithium-ion battery packs was approximately $20 \%$, meaning that on average lithium-ion battery pack prices declined by $20 \%$ a year.

This type of customer economic analysis can help regulators understand when, and among which customers, DPV and DPV-plus-Storage systems may be adopted. It can also be used to understand how customer economics may change over time due to declining technology costs, and or to test the impact of changes to retail tariffs to accommodate DPVplus-Storage customers.

Maximizing On-site Generation with Coupled DPV - Under the current net energy metering regimes for rooftop solar prevalent in most Indian states, the distribution network is effectively a form of "financial storage", storing exported DPV kWhs for the customer for close to free. However, as Indian states transition away from net energy metering schemes and toward net billing schemes, there may be an increased economic incentive for DPV 
customers to deploy a coupled behind-the-meter storage system. Most net billing schemes reward grid injections of DPV generation at a sell rate that is lower than the retail rate for electricity (e.g., at the prevailing DISCOM average power purchase cost) - this provides an economic incentive for customers to maximize on-site consumption of their DPV, which can be enabled by a co-located storage system. Issues related to net energy metering and net billing and behind-the-meter storage are discussed in Section 4.1.

DISCOM Program Offerings - While not yet established by any Indian DISCOMs to date, in the future, DISCOMs could offer incentive programs to customers which provide a fixed monthly bill credit to customers. This bill credit would be granted in exchange for periodic access to a customer's behind-the-meter storage system, which the DISCOM could use to manage the distribution network. Concepts for such programmatic offerings are discussed in Section 8.5.

Direct Financial Incentives - While not yet an established policy by the central government or in any Indian state, in the future, financial subsidies made available by various governments could serve as a driver of behindthe-meter storage deployment. Financial incentives for behind-the-meter storage are discussed in Section 8.4.

Customer Education and Awareness - Like all distributed energy resources, customer awareness around the benefits and opportunities related to behind-the-meter storage and storage-plus-DPV is a significant factor influencing decisions to install these resources.

\subsection{Storage Market Context: Global and United States}

Around the world, growth in behind-the-meter storage deployment is catalyzed by clean energy and environmental goals, interest in resilience and backup power, and further reductions in storage costs, among other factors. By 2026, Navigant estimates that the annual global market for distributed storage energy systems ${ }^{14}$ will reach 27.4 GW, led by the Asia Pacific and Western Europe regions (Figure 6) (Tokash and Dehamma 2017).

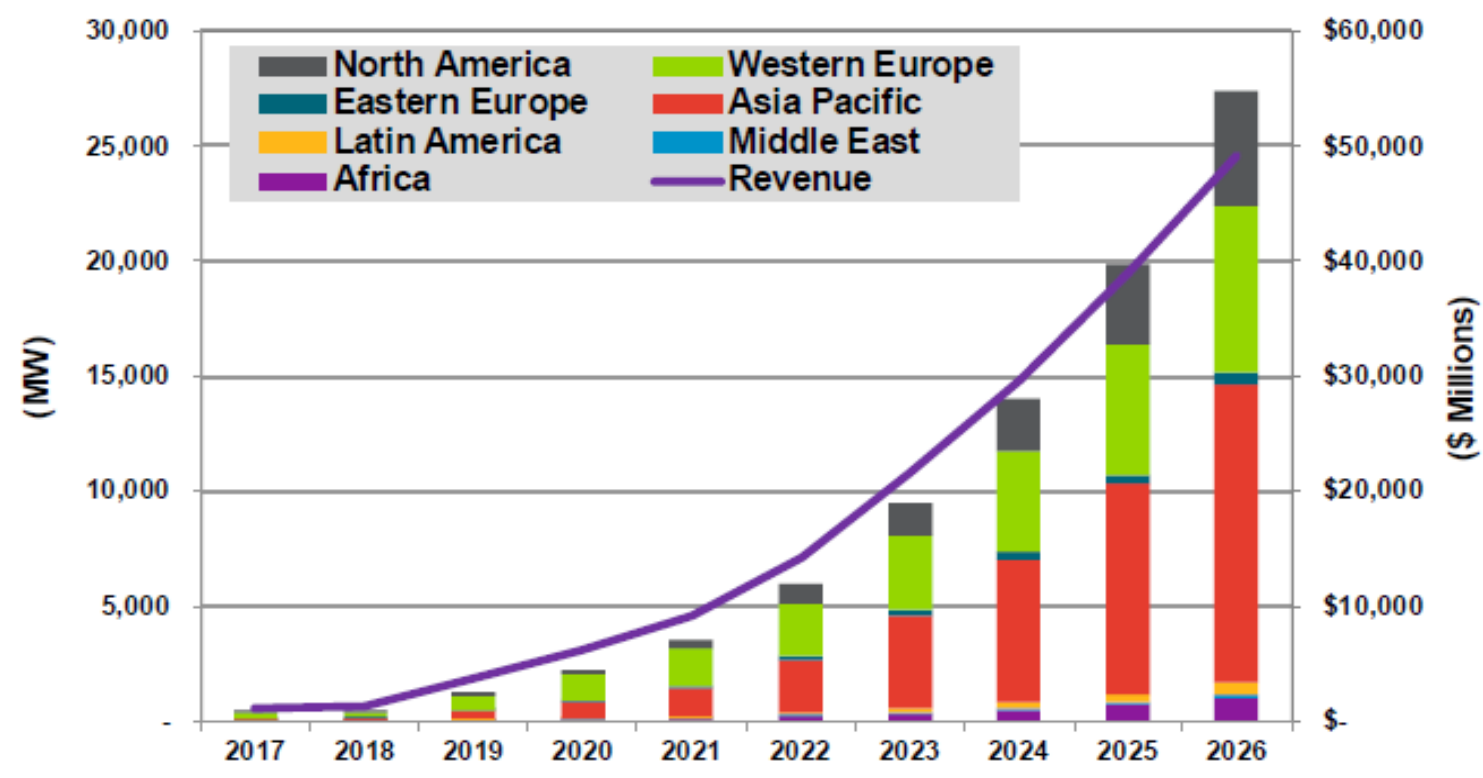

Figure 3. Estimated annual distributed grid and off-grid PV-plus-storage power capacity and expected vendor revenue by region, world markets: 2017-2026

Source: Tokash and Dehamma 2017

Given similar energy governance structures between the United States and India, it is also instructive to explore characteristics of the U.S. battery market. At a high level, the emergence of distributed energy resources (DERs) is reshaping the traditional power sector paradigm in many U.S. states. As distributed solar deployment increases globally, a similar uptake in distributed storage is not far behind. Between Q1-2013 and Q1-2019, the U.S.

${ }^{14}$ Accounting for both distributed-grid-tied and off-grid systems. 
storage market ${ }^{15}$ grew by $1,252.7 \mathrm{MW}^{16}$ of deployed capacity, dominated by front-of-the meter systems, but with notable residential and nonresidential (behind-the meter) additions in more recent quarters. Of the systems installed between Q1-2013 and Q1-2019, 95.8\% of the capacity of systems were of a lithium-ion chemistry type. The growth in capacity of these systems shows no signs of slowing down, with an estimated $16.9 \mathrm{GW}$ of forecasted capacity additions from 2020-2024. The behind-the-meter market segment dominates in terms of number of annual and cumulative systems deployed, with an exponential uptick in behind-the-meter deployments observed since 2017 (Figure 4)(GTM 2019).
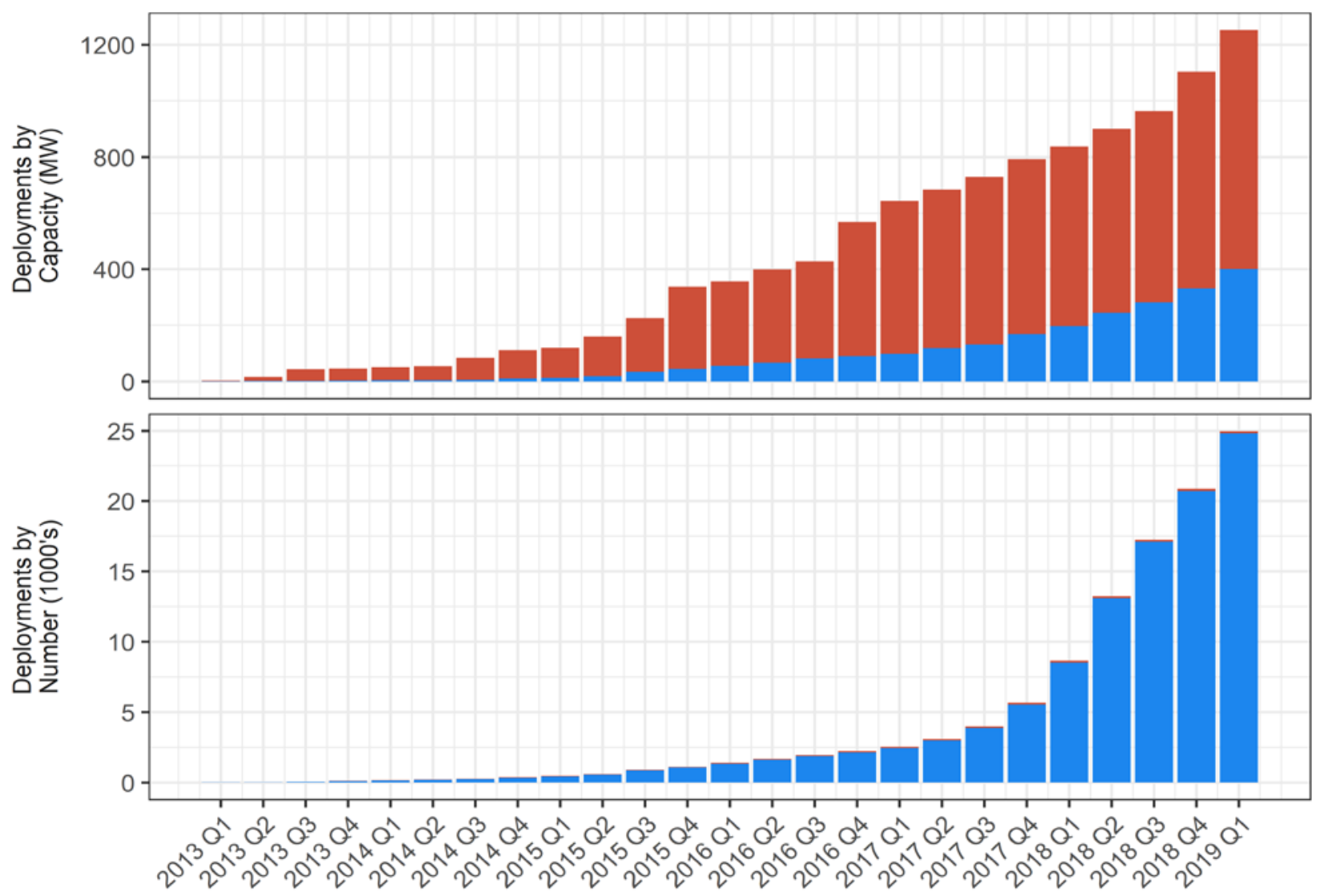

Quarter

Market $\square$ Front-of-the-Meter $\square$ Behind-the-Meter Figure 4. Cumulative U.S. deployment of energy storage by capacity and number of systems for front-of-
the-meter and behind-the-meter systems from Q1 2013 to Q1 2019

Source: GTM 2019

The key drivers of residential and commercial behind-the-meter storage deployment in the United States include increasing customer interest in self-consumption of distributed generation and resilience, metering and billing arrangements, ${ }^{17}$ and retail tariff design changes, such as net energy metering (NEM) and time-of-use (TOU) rates, that help to drive customer bill savings, grid service opportunities, policy and regulatory mandates and incentives, and system cost reductions (GTM 2019). The U.S. context is extremely diverse with respect to these drivers, as well as market organization (ranging from fully deregulated retail and wholesale power markets in Texas to

${ }^{15}$ Defined by Mackenzie Wood as "Electrochemical (batteries) and electromechanical technologies, excluding pumped hydropower, are included in the historical deployment and forecast data." (GTM 2019).

${ }^{16}$ Assuming no retirements between 2013-2018.

${ }^{17}$ Metering and billing arrangements define how consumption and generation-related electricity flows are measured and credited, and include strategies such as NEM, net billing, and buy-all, sell-all. This will be discussed in more detail in Section 4. 
vertically integrated utilities in Hawaii) and state and local policy priorities (with several states having targets for storage deployment and/or 100\% clean energy targets). This wide range in drivers and approaches to integrating and incentivizing storage assets has led to a diversity of deployment results across states, with behind-the-meter storage systems more concentrated than front-of-the-meter installations (Figure 5). California and Hawaii have emerged as perhaps the most vibrant markets for behind-the-meter storage adoption, with both jurisdictions seeing significant deployment of both grid-tied DPV and DPV-plus-storage systems. This diversity of state actions and levels of deployment in the United States provides a nearly comprehensive study of the variety of means available to utilities and policymakers to incentivize and integrate storage and DPV-plus-storage systems. Looking across all states, it is evident that state policy and regulation can strongly influence battery deployment.

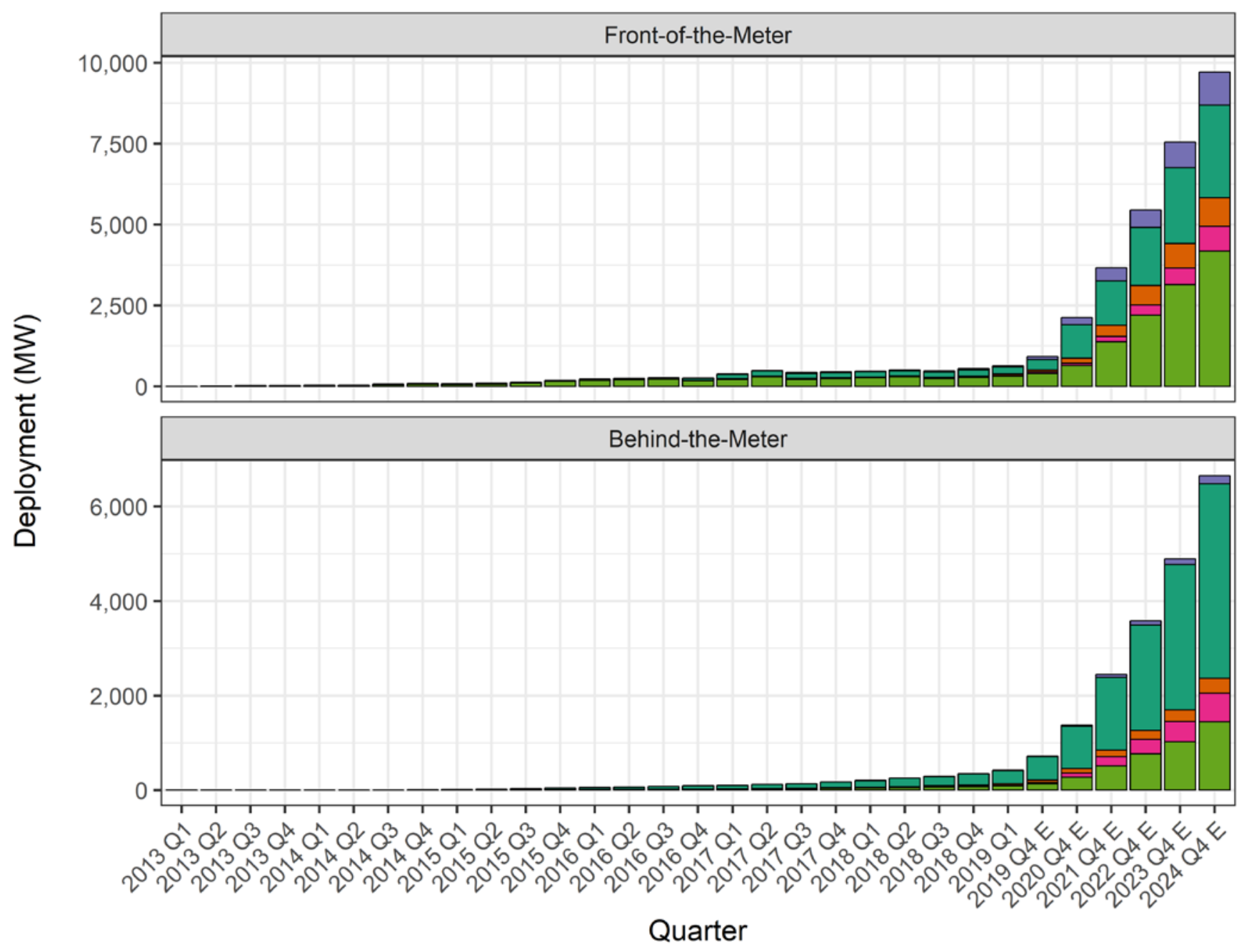

State $\square$ Arizona $\square$ California $\square$ Hawaii $\square$ New York $\square$ All Others

Figure 5. U.S. state deployment of energy storage by capacity of behind-the-meter and front-of-the-meter systems, historical and project

Source: GTM 2019 


\section{Approaching DPV-Plus-Storage Program Design}

The remainder of this report proposes a process for regulators to develop comprehensive programs for behind-themeter DPV-plus-storage systems in order to achieve specific objectives. Unlike large, centralized assets, DPVplus-storage deployment can comprise of millions of systems being installed independently according to customer preference, with limited control and oversight by DISCOMs or regulators. As such, it can be more difficult to ensure that these systems do not compromise the reliable functioning of the power system and that they contribute to broader power system objectives. Furthermore, the ability of such systems to achieve certain objectives may depend on the coordination of upfront investments, such as sufficient communication infrastructure or advanced inverter and meter deployment, that may be prohibitively expensive to retroactively ensure once the systems are already installed.

The process outlined in this report consists of several steps, which are displayed as distinct decisions for the purpose of organizing the report, but are very much interrelated and interdependent in reality. Although this process is written explicitly for DPV-plus-storage, the basic steps could also be adapted for other distributed energy resources. It is also important to note that for many jurisdictions, a regulatory framework already exists for behind-the-meter DPV resources, in which case the primary task for regulators will be to incrementally adjust existing regulation to include storage, as opposed to developing an entirely new framework.

Throughout the report, jurisdictional case studies are used to highlight how governments, utilities and regulators have already begun approaching these issues. These case studies are primarily taken from the United States, as the United States and India share similar state-centric governance structures in the electricity sector, and the majority of states in both nations also feature (or at one time featured) net energy metering (NEM) as a core distributed generation policy. Given these similarities, this report offers potentially transferrable lessons to the Indian context via the U.S. experience.

\subsection{The Program Design Process}

At a high level, designing a regulatory framework that aligns DPV-plus-storage deployment with larger policy objectives requires thoughtful deliberation across a range of technical and economic issues. While this report attempts to segment many of these issues into distinct topics to enhance reader understanding, DPV-plus-storage regulatory issues are all closely integrated, and design decisions on a single aspect often have broader implications. Furthermore, while the program design process presented in this report offers a sequential framework for considering DPV-plus-storage program design decisions, in reality, the development of various technical and economic regulations can - in many cases - be undertaken in parallel.

With those qualifications, Figure 7 presents a high-level process for how regulators can sequentially approach the design of DPV-plus-storage regulatory frameworks. As a first step, developing and prioritizing clear regulatory objectives - which may be influenced by, aligned with, or directly derived from stated public policy goals - can help guide individual decisions on designing regulations (Section 3). Next, regulators can consider the appropriate compensation mechanism offerings for DPV-plus-storage systems that attempts to address and balance objectives related to fair customer compensation and utility cost recovery, as well as the desired role of DPV-plus-storage in the power system (Section 4). Thereafter, rules governing the metering and technical configuration requirements can be designed to ensure the desired compensation mechanism can be implemented (Section 5). Then, the overarching interconnection process can be designed to offer a safe and orderly procedure for DPV-plus-storage systems to apply for interconnection, as well as to provide technical guidance to installers on the various regulatory requirements that must be complied with (Section 6). Next, regulators may need to consider and account for local permitting requirements (and associated safety codes) that must be complied with to deploy behind-the-meter storage systems (Section 7). Finally, because all regulatory processes exist within a broader policy and market context, common policy and market instruments and approaches related to DPV-plus-storage are discussed briefly in Section 8. Importantly, steps can also interact in other ways. For example, compensation mechanisms can be designed to accommodate existing metering configurations. After the steps Figure 7 are discussed in detail, Section 9 offers a summary of key regulatory considerations for DPV-plus-storage systems. 
Drlves design of all technical and economic aspects of storage-plus-DPV regulations
Step II: Design Compensation Mechanism

(Section 4)

$$
\begin{array}{l|l|}
\begin{array}{l}
\text { Addresses objectives } \\
\text { related to role of } \\
\text { storage-plus-DPV in power } \\
\text { system and issues of fair } \\
\text { compensation and utility } \\
\text { cost recovery. }
\end{array} & \begin{array}{l}
\text { Step III: Design Mete } \\
\text { Technical Configura }
\end{array} \\
\begin{array}{l}
\text { Enables implementation of } \\
\text { compensation mechanism }
\end{array} \\
\begin{array}{l}
\text { technical configuration } \\
\text { requirements }
\end{array} & \begin{array}{l}
\text { Addresses objectives } \\
\text { related to role of } \\
\text { storage-plus-DPV system, } \\
\text { metering costs and } \\
\text { compensation mechanism } \\
\text { integrity }
\end{array} \\
\hline \begin{array}{l}
\text { Drlves interconnection } \\
\text { requirements }
\end{array}
\end{array}
$$

Step III: Design Metering and

Technical Configuration Requirements

\section{Step IV: Design}

Interconnection Process

Enables implementation of metering and technical configuration requirements

Addresses objectives related to utility

administrative burdens and reasonable interconnection costs/timelines
(Section 5)

(Section 6)

\section{Step V: Consider}

Account for relevant local construction permitting

and safety requirements*

"not direct responsibility of regulator Local Permitting Issues (Section 7)

\section{Figure 6. Approaching program design for DPV-plus-storage systems}

Source: Original [IIlustration by Christopher Schwing, NREL]

\subsection{Customization Dimensions for Program Design}

Similar to grid-tied distributed generation systems without storage, it may be worthwhile for regulators to consider implementing distinct compensation mechanisms and technical requirements for different segments of the retail customer base to better balance various regulatory objectives. For instance, it is common in clean distributed generation programs to offer more generous compensation levels with less rigorous metering requirements for smaller capacity DPV systems and/or residential customers to improve the economics of smaller-scale systems with a lower magnitude of technical and financial impacts on the utility system at lower deployment levels.

The various customization dimensions detailed in Table 2 are not mutually exclusive and can be implemented in parallel as deemed appropriate by the regulator for distinct compensation mechanism offerings and associated metering and technical configuration requirements. 


\section{Dimension Description}

Customer Class

Offering distinct compensation mechanisms to customers, using distinct retail tariffs may be an effective strategy for balancing utility and customer interests in a more customized manner, as customers with distinct retail tariffs have accordingly distinct financial impacts on utilities.

Voltage Implementing distinct metering, technical configuration, or interconnection requirements for system Interconnection Level connecting to the network at distinct voltage levels may be an effective strategy to ensure that requirements are designed with an appropriate level of rigor for the expected impacts and potential service offerings of the DPV-plus-storage system.

System Capacity System capacity can be based on the capacity of the DPV system, the storage system output, the combined capacity of both systems, or the maximum output from the system the grid will see based on limitations imposed on the system (e.g., inverter software that limits the maximum output). Offering distinct requirements based on system capacity can be useful for delineating both compensation levels and grid requirements, with larger systems typically being subject to a more rigorous review process. In India, system capacity limits are often stated as a percentage of contracted demand and/or sanctioned load for particular customer classes.

Consumption Magnitude

Geography

A monthly or annual consumption segmentation is also possible, using historical consumer consumption data to calculate a rolling average metric for kWh usage, offering distinct compensation mechanisms for various tiers of consumption. This approach might be useful should there be a desire to offer more generous compensation to lower consumption customers, as the magnitude of their expected utility financial impact would be smaller due to their lower overall energy needs.

Different regions or service territories within a country may have distinct technical or economic considerations that merit different approaches to regulation. For instance, geographically remote and/or off-grid systems may merit separate regulatory treatment compared to customers in urban centers.

Local Grid Characteristics

The characteristics of the local distribution system where a system may be deployed could merit a customized approach to creating regulatory requirements and compensation mechanisms for DPVplus-storage systems. For example, in areas with distribution feeders that regularly experience power quality issues, certain technical configuration requirements and compensation mechanisms may be appropriate to ensure that batteries can provide power quality services and receive a fair compensation for doing so. This approach may be good practice to support efficient technical integration of DPV-plus-storage resources, but also requires extremely clear technical criteria and a predictable process to regularly update regulatory requirements for newly interconnecting resources.

\subsection{Other Relevant Regulatory Tools}

\subsubsection{Deployment Caps and Tools}

Program caps are limits on the total capacity or number of systems eligible for a DER program, or can be stated as a total budget allotted for a DER program, regardless of any individual system's size, and can be an important tool in the development and deployment of rebate and financial incentive programs, and even compensation mechanisms and tariff offerings. Program caps are a simple but effective tool to ensure cost containment for utilities administering the program, as well as to limit the impact such DPV-plus-storage systems may have on a utility's revenue, or on the level of cross-subsidization borne by nonparticipating customers. These caps can be differentiated among subregions or staggered across periods of time. Program caps can be calculated in a number of ways, including as an absolute capacity limit or as a percentage of peak demand. Program caps can also be used to trigger changes in the program, and in some jurisdictions in the United States, program caps are used to initiate proceedings to reevaluate incentive programs and compensation mechanisms (NREL 2019). Most often these caps are established upfront to clearly state program goals, and they can be adjusted over time as different deployment caps are met.

Deployment-based payment adjustments are a means to adjust compensation mechanism offerings as cumulative deployment of a DER technology increases and have been commonly used in the context of DPV. As opposed to a program cap, which more plainly sets a ceiling for deployment levels, deployment-based payment adjustments 
might reduce the DPV-plus-storage sell rate or make adjustments to retail tariffs once certain deployment levels are achieved. For instance, Germany's feed-in tariff program set a predetermined schedule for how the sell rate ${ }^{18}$ for DPV-plus-storage would decrease as cumulative deployment increased (Bundesnetzagentur 2019). In Israel, the regulator implemented a new tariff component on retail customers once cumulative solar capacity (including both utility-scale and distributed resources) in the country achieved 1.8 GW of deployment to reflect growing grid integration costs (PUA 2014).

System size caps are absolute limits on the size of individual distributed generation (DG) or storage systems, and are typically stated in terms of capacity (e.g., $\mathrm{kW}$ or MW) or an annual ratio of DG production to customer load (known colloquially as the PV-to-Load ratio). Capacity-based caps tend to be implemented to limit compensation mechanism eligibility (e.g., NEM is only available for systems under $100 \mathrm{~kW}$ ) and to ensure that interconnection requirements are appropriately rigorous given the size of the system (e.g., systems under $10 \mathrm{~kW}$ are granted a fasttrack screening process). System size caps based on the PV-to-Load ratio tend to be implemented to encourage self-consumption behavior (as opposed to utilizing DPV-plus-storage customers as grid-interactive resources). As mentioned in the previous section, system size caps are a useful tool for segmenting the retail customer base to offer customized compensation mechanisms and interconnection requirements.

\subsubsection{Alternative Ratemaking Paradigms}

Revenue decoupling is a regulatory strategy to address utility disincentives to support energy efficiency and distributed energy resource programs which reduce utility sales. Specifically, regulators sever the connection between utility revenues and the volume of retail electricity sales. Tariffs are instead designed to ensure that utility will earn sufficient revenue to cover network and energy procurement costs. Revenue decoupling is a substantial departure from traditional cost-of-service regulatory approaches and is not a decision to be taken lightly. This topic has been discussed at length in the literature - see e.g., Lazar et al. (2016).

Performance-based regulation represents another significant shift in utility regulation and ratemaking. Performance-based regulation provides a framework to: (1) set utility goals and targets; (2) measure utility performance in achieving these goals and targets; and (3) link utility performance to utility compensation, including investor returns and executive compensation. By setting predetermined metrics for performance, regulators can create financial incentives for activities that utilities might not normally be motivated to pursue, such as the streamlining the interconnection of DPV-plus-storage resources. Similar to revenue decoupling, shifting toward the use of utility performance incentive mechanisms is a significant change. While quite relevant for more advanced jurisdictions facing large quantities of distributed energy resources, it is outside the scope of this report. For more information, see Littell et al. (2017) and Whited, Woolf, and Napoleon (2015).

\footnotetext{
${ }^{18}$ We define a sell rate as the level of compensation a DG system owner receives for electricity exported from the DG system to the grid.
} 


\section{Step I: Develop and Prioritize Regulatory Objectives}

\section{Key Insight for Indian Stakeholders}

This chapter focuses on how regulators can decide on specific regulatory objectives they would like to achieve through DPV-plus-storage systems, as well as the importance of prioritizing these objectives. These objectives are categorized and discussed in-depth to help regulators decide which objectives they may be most interested in pursuing. Importantly, regulators can use the customization tools described in the previous report to help balance the sometimes conflicting needs of DISCOMs and customers, as well as to achieve multiple power system objectives.

In India, although government targets for DPV deployment and rural electrification have been established, there has been limited development to-date in terms of fully activating the latent potential of DPV-plus-storage systems to achieve multiple power system objectives. Most of the deployment has been driven by larger C\&I customers for bill savings purposes, and there has been little coordination between these customers and the DISCOMs to maximize these systems' utility to both parties. Currently storage systems are encouraged or required to operate in an entirely passive manner, e.g. to meet load during outages, and there has been limited efforts to explicitly encourage the deployment of these systems or to use these systems to achieve specific objectives, especially for more grid-interactive roles.

As a first step, Indian state and national government leaders can articulate policy and regulatory objectives to take full advantage of the opportunities that behind-the-meter battery systems can bring to customers, utilities, and the broader power system. Currently, only a handful of Indian states mention behind-the-meter storage in their regulations, including Assam, Jharkhand, Maharashtra, Karnataka, and Rajasthan (AERC 2015; JSERC 2015; KERC 2016; RERC 2016; MERC 2015). While these states restrict storage systems from discharging to the grid during outages, only Assam explicitly restricts grid discharges during normal operations. This leaves room for state electricity regulatory commissions (SERCs) to clearly articulate their objectives and desired-use cases for storage systems (including the extent to which grid-interactive behind-the-meter storage systems are desired), which will help provide guidance for developers, DISCOMs, and customers.

SERCs can pursue a variety of objectives related to DPV-plus-storage systems that reflect their specific power system conditions and existing policy environment. For instance, in Jharkhand, which is land constrained and experiences relatively high outage rates, regulatory objectives may involve using DPV-plus-storage assets to contribute to power system reliability or encourage backup power applications. Conversely in Gujarat, which has higher reliability, regulators may be more concerned in meeting ambitious renewable energy deployment targets. Systems focused on mitigating distribution-level power quality issues may wish to activate DPV-plus-storage for its passive grid support functions (see Section 6.2) whereas systems regularly experiencing periods of stresssuch as high afternoon peaks or rapid evening ramp rates - may wish to encourage certain technical configurations (see Section 5.1) and/or utilize tariff design to encourage DPV self-consumption or exports during specific time periods (see Section 4.2). Desires for greater DPV-plus-storage system transparency and ease of monitoring, meanwhile, could lead to more extensive metering requirements. To help balance these objectives with consumer and utility needs, Indian policymakers have a wide variety of tools available to customize rules and regulations along a variety of dimensions (see Sections 2.2 and 2.3). Regardless of the objectives regulators may choose to emphasize, these objectives will have important downstream effects on other decisions regarding compensation mechanism design (see Section 4), technical configuration (see Section 5.1) and metering requirements (see Section 5.2), and interconnection processes (see Section 6).

DPV-plus-storage may variously inform and be the target of a diverse range of underlying regulatory objectives. These objectives influence design decisions pertaining to compensation mechanisms, metering requirements, technical configuration requirements, and interconnection processes. Thus, developing a set of clearly articulated regulatory objectives can serve as a useful first step for regulators. Importantly, most objectives are neither mutually exclusive nor binary; however, some objectives may conflict with others. Facilitating upfront dialogue to help weigh, balance, and prioritize objectives can facilitate faster decision-making. Potential objectives for DPV-plus-storage programs are presented below. 


\subsection{Approach to DPV-Plus-Storage Market}

Depending on the relevant set of statutory mandates, policy guidance, and/or legal authorization that a regulatory agency has, regulators may exhibit a range of perspectives toward DPV-plus-storage programs. These might include ${ }^{19}$ :

- Constrain DPV-plus-storage deployment-Some regulators may be unfamiliar or concerned about DPV and DPV-plus-storage systems, and wish to constrain deployment through direct actions or deliberate inaction.

- Enable DPV-plus-storage deployment-Most regulators are typically mandated with an enabling role for DER programs that focuses on motivating the utility to facilitate a safe and orderly interconnection process, while also preserving fairness among participating and nonparticipating customers and preserving utility revenue sufficiency.

- Accelerate DPV-plus-storage deployment-Some regulators with more ambitious mandates or specific objectives in mind for the desired role of DPV-plus-storage may wish to see utilities play a role in accelerating the development of the DPV-plus-storage market.

\subsection{Desired Role of DPV-Plus-Storage Systems}

DPV-plus-storage systems can play a variety of roles in power systems, from exclusively serving customer needs, to being fully grid-interactive assets that provide a range of services to the distribution and/or transmission system, with many options in between.

- Encourage self-consumption for DPV-plus-storage systems - Regulators may wish to see DPV-plusstorage systems deployed to primarily or explicitly serve a customer's own energy demand, and/or for backup purposes during grid outages. They may also wish to reduce the likelihood or scale of net exports to the grid and/or mitigate major peaks in grid injection and withdrawal, which can cause challenges for certain utilities and their power systems. In practice, encouraging self-consumption for DPV-plus-storage systems may mean discouraging the over-dimensioning ${ }^{20}$ of storage and/or DPV assets through specific regulations. At a high level, an objective to encourage self-consumption from DPV-plus-storage systems will have implications for the technical configuration requirements of systems and may prove consequential for the future ability of DPV-plus-storage systems to become grid-interactive.

- Utilize DPV-plus-storage to manage system peaks-Regulators may be particularly interested in enrolling DPV-plus-storage customers to manage system peaks, particularly in regions or on specific feeders that regularly experience steep ramps in net load and/or load shedding. This can be enabled through retail tariff design that encourages reductions in grid consumption and/or grid injections during times of peak demand, as well as demand response programs that rely on customer-sited storage. Utilizing DPV-plus-storage to manage system peaks is a commonly discussed form of grid interactivity.

- Enable full grid interactivity of DPV-plus-storage systems - Regulators may wish to enable and/or preserve the option for DPV-plus-storage systems to provide a range of valuable grid services to the power system. Such grid interactivity could be as simple as allowing DPV-plus-storage customers to participate in energy arbitrage activities, such as by reducing demand, or increasing DPV-plus-storage exports during peak demand periods in response to TOU price signals (see previous objective). It could also involve the provision of a range of other services (e.g., congestion management, frequency regulation), enabled through "virtual power plants," where third-party aggregators sell grid services from multiple distributed systems directly to distribution companies and/or on wholesale power markets. Encouraging grid interactivity can spur the deployment of DPV-plus-storage systems by increasing the value streams available to them, while helping to contribute to power system operations by aligning individual customer behavior with the broader needs of the power system. In any case, the extent of desired grid interactivity has significant implications for a wide range of regulatory decisions, including those relating to compensation mechanism design, metering, as well as technical configurations. In particular, decisions related to whether or not the storage system should be

\footnotetext{
${ }^{19}$ This framing of approaches to storage-plus-DPV loosely follows that provided in_(Rickerson et al. 2014).

20 "Over-dimensioning" refers to the oversizing of DG (and storage) systems relative to the energy needs of the individual customer. This is particularly a concern in settings which offer more generous compensation rates for clean distributed generation.
} 
allowed to charge from and export to the power system (see Section 5.1) have important implications for DPV-plus-storage grid interactivity objectives.

\subsection{Fairness and Equity Considerations}

Regulators may be presented with various fairness and equity considerations as they deliberate program design options. Some common objectives pertaining to fairness and equity include:

- Balance the financial interests of utilities, DPV-plus-storage customers, and nonparticipating customers - Similar to dialogues surrounding DPV, regulators designing DPV-plus-storage programs typically attempt to balance the financial interests of utilities, adopting customers, and nonadopting customers, while attempting to not upset existing balances of cross-subsidization between customer classes. In practice, this means regulators must deliberate how to ensure that utilities' fixed costs continue to be adequately recovered through electricity sales, even as these sales are reduced through DPV-plus-storage, as well as through customer self-consumption occurring under any self-supply scheme. ${ }^{21}$ Regulators must also consider how to protect nonadopting customers from cost-shifting if utilities are granted retail tariff increases to recover lost revenue due to DPV-plus-storage deployment. The balance that regulators ultimately strike will have implications for compensation mechanism design, and in particular the design of retail tariffs and metering and billing arrangements. Related to this objective, regulators may also wish to reduce the likelihood of customers deciding to become entirely self-reliant and disconnecting from the grid $^{22}$ through providing more attractive compensation mechanisms or implementing certain technical configuration requirements which encourage the enrollment of DPV-plus-storage customers as grid resources.

- Create fair and reasonable metering and interconnection requirements for customers and utilitiesComplying with metering and interconnection requirements typically comes at an incremental cost that is typically born by DPV-plus-storage customers. As these incremental costs can reduce the economic viability of DPV-plus-storage systems, regulators can help to ensure that they are of an appropriate scale relative to the overall system's cost. Furthermore, in the event that regulators are attempting to mitigate a particular undesired behavior with additional metering infrastructure (e.g., energy arbitrage activities using the storage device), they might aim to ensure that the cost and/or administrative burdens associated with these requirements are commensurate with the scale of problem they are trying to mitigate, so as to not enforce an undue burden on DPV-plus-storage customers.

- Preserve the integrity of DPV compensation mechanisms - Compensation mechanisms have historically been implemented to reward exported energy that is generated from eligible DG systems (e.g., DPV). Some regulators may wish to place an increased emphasis on ensuring that grid-supplied energy later exported by the storage system does not yield a financial reward that is intended for clean DG (see Section 5.3). ${ }^{23}$ The extent of a regulator's emphasis on this goal will influence decisions surrounding compensation mechanism design, metering, and technical configurations.

\subsection{Program Administration}

Regulators, along with utilities, may be interested in ensuring that program administration tasks are not overly cumbersome or expensive for utilities and customers. Some common objectives in this realm include:

- Reduce administrative requirements for utilities - The implementation of DPV-plus-storage programs may drive new administrative requirements for utilities, in particular associated with processing interconnection applications and implementing new billing procedures. Different approaches to designing

\footnotetext{
${ }^{21}$ In the realm of distributed energy resource program design, regulators are frequently asked to balance the interests of utilities who are concerned about revenue sufficiency and customers/ developers who are concerned with their ability to purchase or offer economic DPV systems. For instance, the Maharashtra Electricity Regulatory Commission has been asked by local solar industry advocates to investigate new volumetric grid service charges proposed by a local DISCOM, MSEDCL.

${ }^{22}$ In this realm, the terms grid defection and load defection are commonly invoked to describe the process of customers choosing to meet the entirety or majority of their energy demand through on-site DERs such as rooftop solar and behind-themeter storage for primarily financial reasons. Of course, in countries with highly unreliable grids, customers may have little choice but to use other means for their energy needs.

${ }^{23}$ Importantly, such customer behavior could ultimately be quite desirable to regulators depending on the specifics of their power system.
} 
interconnection processes or metering requirements may have implications for the administrative workload for utilities. For instance, encouraging utilities to transition to standardized online portals for processing interconnection applications can drastically reduce the amount of time and labor needed for each application compared to processing applications individually by hand (Peterson and Reiter 2017).

- Avoid new requirements on existing systems - Changing metering or other technical requirements after systems are already installed can be expensive and administratively burdensome. Regulators may wish to consider decisions surrounding metering requirements, technical configurations, and interconnection requirements in light of not only the current role of DPV-plus-storage systems on the power system, but also how that role may change over time, particularly if that future role may involve more grid interactivity. Along similar lines, regulators may wish to design rules such that existing regulations for grid-tied DPV systems also apply to DPV systems that are retrofitted with storage, which can reduce uncertainty and burdens for customers seeking to add storage to their existing DPV systems. Particularly in jurisdictions with large amounts of existing grid-tied DPV systems, creating clear and streamlined "on-ramps" for customers adding storage to an existing DPV system may be an important regulatory consideration.

\subsection{Transparency, Monitoring, and Safety}

Regulators, along with utilities, may be interested in promoting additional visibility into the operation of DPVplus-storage systems to support greater safety and reliability in the power system, policy compliance, and/or improved power sector planning exercises. This is typically achieved through the deployment of supplementary metering and information and communication technologies that collect, store, and transmit relevant real-time power system operational data, which comes at an additional cost to customers and/or utilities. Thus, the following objectives are often in tension with objectives to minimize unfair cost burdens.

- Promote real-time intelligence and transparency-Regulators may wish to ensure utilities have access to high quality operational data and/or the ability to remotely island DPV-plus-storage systems during outages. Having access to data about - and perhaps even remote control over - the DPV-plus-storage system can help ensure reliable service delivery by enhancing operational awareness on individual feeders of the distribution network, while reducing safety risks to utility line workers. This objective may influence decisions relating to communications and control requirements that are placed on DPV-plusstorage systems through metering configuration rules and interconnection requirements.

- Track clean energy production-For regulatory compliance with clean energy goals, such as mandates and Renewable Portfolio Standards (RPS) (see Section 4.3), some regulators may wish to have increased visibility into the production of the DPV component of the DPV-plus-storage system. This objective may influence metering requirement decisions.

- Track users' gross electricity demand-Understanding a customer's electricity demand patterns independently of the DPV-plus-storage system can help inform utility planning and operations and may be a stated objective for regulators and/or utilities. Like the previous objective, this may influence metering requirement decisions.

\section{Jurisdiction Examples}

California-Policymakers in California have long made the acceleration of distributed generation a priority in their state for an evolving set of reasons. In 2000, the Self-Generation Incentive Program (SGIP) was developed to reduce peak demand during the California electricity crisis. In 2009, the program was updated with the explicit goal of reducing greenhouse gas emissions as a part of California's evolving environmental ambitions. Originally designed to help subsidize the costs of distributed generation, the program was again updated in 2017 to pivot more towards encouraging the deployment of energy storage, whose expanded use the legislature found could:

- "assist ... in integrating increased amounts of renewable energy resources ... in a manner that minimizes emissions of greenhouse gases."

- "optimize the use of the significant additional amounts of variable, intermittent, and offpeak electrical generation from wind and solar"

- "reduce costs to ratepayers ..."

- "reduce the use of electricity generated from fossil fuels to meet peak load requirements ..." 
- "provide the ancillary services otherwise provided by fossil-fueled generating facilities."

The SGIP represents only a small portion of the efforts that California regulators and policymakers have undertaken in order to ensure policy objectives. It is, however, representative of the evolution of priorities for stakeholders in California and shows how programs can be updated in response to changing power market contexts (Skinner 2010; CPUC 2019). (See 'Jurisdiction Examples' in Section 8.4 for more information on the Self-Generation Incentive Program).

Hawaii-In 2001, Hawaii's Public Utilities Commission directed the islands' utilities (HECO, MECO, HELCO, KIUC) to initiate a net energy metering scheme as a means of encouraging distributed generation, meeting environmental goals and reducing reliance on expensive imports which Hawaii requires to meet electricity demand. The NEM program was an effective means to encourage the deployment of distributed generation and from 2001 to 2015 , over 60,000 systems for a total of nearly $500 \mathrm{MW}$ of PV were installed on Hawaii's islands (HECO 2020). Given the relatively small size and isolated nature of Hawaii's power system, along with the excellent solar resource in the area, exports of electricity from behind-the-meter rooftop systems soon led to concerns of excess energy in the day and reverse power flow on circuits.

At this stage, the existing suite of program objectives in Hawaii shifted to include the mitigation of grid integration issues as a key priority. This prompted regulators and the utilities to end NEM in 2015 in favor of alternative tariff options that would incentivize customers to shift solar exports outside of daytime hours, either by self-consuming their energy or using storage to export that power later in the day (for more information on Hawaii's tariff options, see 'Jurisdiction Examples' throughout Section 4). Evolving power system contexts in Hawaii, combined with an evolution in broader policy objectives (including Hawaii's decision to achieve $100 \%$ electricity generation from locally produced renewable energy), have led to changes in program design for DPV and DPV-plus-storage. These changes have ensured that customers can still pursue distributed generation without compromising, and in fact contributing to, power system reliability and objectives. 


\section{Step II: Design Compensation Mechanism}

\section{$\underline{\text { Key Insight for Indian Stakeholders }}$}

This chapter focuses on the development of compensation mechanisms to incentivize desired behavior from customers and DPV-plus-storage systems. It begins with a comparison of two billing and metering arrangements, Net Energy Metering and Net Billing, and the consequences for the deployment and operation of DPV and DPVplus-storage systems. Then a detailed discussion of the myriad of retail tariff options available to regulators is presented, with a focus on how these tariff elements can drive different customer behaviors to the benefit of DISCOMs. Finally, time-invariant and time-variant sell rates are briefly discussed.

In India, most customers are subject to flat (time-invariant) volumetric rates, with some larger customers subject to Time-of-Day rates and demand charges. Retail tariffs in India also include inclining volumetric blocks, where customers pay higher electricity rates if they consume more. This has led many smaller consumers, such as agricultural or residential consumers, to pay rates that are cross-subsidized by larger C\&I customers. This crosssubsidy scheme is taken as a social necessity to encourage the electrification of the country but has also encouraged larger customers to reduce their bills through the deployment of DPV and DPV-plus-storage systems. As these larger consumers begin reducing their demand, it places increased pressure on already financiallystressed DISCOMs who may already struggle to ensure revenue sufficiency. The primary compensation mechanism for DPV in India is Net Energy Metering, although some states have begun transitioning to Net Billing. In most cases there is limited discussion on the eligibility for DPV-plus-storage systems to qualify for compensation under NEM or NB, which may inadvertently discourage pairing PV and storage.

As some Indian states begin transitioning toward Net Billing schemes, the economics of DPV-plus-storage systems may increase relative to standalone DPV systems. Although two states in India have moved away from conventional NEM (Gujarat and Maharashtra), most states currently feature a NEM framework for DPV that effectively activates the grid as a "financial battery" for customers. While NEM is an effective incentive for gridtied DPV systems, access to such a free financial battery is likely more favorable to customers than the capital expenditure associated with a new behind-the-meter battery system (see Section 4.1.1). Furthermore, from a policy perspective, NEM programs are typically premised on the assumption that energy injected to the grid comes directly from a distributed generation system rather than from an energy storage device. Thus, absent changes to retail rate design or metering and billing arrangements, current state NEM regimes would not in themselves facilitate the coupling of DPV with storage systems; however, the FOR's Draft Model Regulation for Grid Interactive Distributed Renewable Energy Sources document describes a potential net billing arrangement for SERCs to consider (FOR 2019). Adopting such an arrangement would effectively increase the value of DPV self-consumption relative to DPV exports and may help to financially incentivize DPV-plus-storage systems, though it would concurrently reduce the financial returns of DPV investments.

Relatedly, alternative tariff designs could further incentivize the deployment of DPV-plus-storage systems in India (see Section 4.2). As noted above, many customers are billed on time-invariant retail rates, which provide little opportunity for customers to reduce their bills through storage systems (see Section 4.2.1.1). Alternative retail tariffs designed specifically for DPV-plus-storage customers - featuring structures such as volumetric timevariant energy rates (e.g., time of day) (see Section 4.2.1.2) or demand charges (both time-invariant and timevariant) (see Section 4.2.2) - could incentivize the coupling of storage and solar, as well as help align customer behavior with system needs. Considerations for placing customers on more advanced tariff structures must be balanced against the cost of new metering and billing infrastructure, as well as the customers' ability to meaningfully alter their consumption in response to price signals from the power system, as many smaller customers may be unable to change their demand to take advantage of new tariffs. 
Zinaman et al. (2017) define a grid-connected DG compensation mechanism as compromising three primary components:

1. Metering and billing arrangement-The first aspect defines how consumption and generation-related electricity flows are measured and credited. This section will focus on net energy metering and net billing schemes for DPV-plus-storage customers;

2. Retail tariff design-This aspect defines the retail tariff structure and exact purchase rate the DG system owner must pay for electricity from the grid, and thus helps defines the potential for customer bill savings. This section will discuss how various retail tariff elements incentivize certain behaviors from the system owner and how these elements influence the relative economics of DPV-plus-storage systems versus standalone DPV systems; and

3. Sell rate design - The final element defines the exact level of compensation a DG system owner receives for electricity exported from the DG system to the grid. ${ }^{24}$ This section will provide a brief discussion on how the level of compensation a system receives for energy exports can impact system economics.

In general, compensation mechanism design decisions have broad implications, spanning the deployment and operational behavior of DG assets, customer bills, and utility revenue collection, as well as metering setups, system technical configuration requirements, and interconnection processes. Compensation mechanisms for DPVplus-storage comprise the same three basic elements as other DG resources. While they share a similar set of implications, there are some important key differences to consider, which are detailed throughout this section. It is also important to note that although these issues are represented here as distinct topics, in reality they are all closely integrated with one another and cannot be considered in isolation. They have been separated in this report to enhance reader understanding, but the relationships between these issues will be discussed throughout the subsequent sub-sections.

As well, regulatory objectives and market context often influence compensation mechanism design and its timing. Solar penetration levels, grid management issues, storage deployment mandates or goals (see Sections 8.1 and 8.2), DER technology costs, utility cost recovery and cross-subsidization considerations, and low-income customer concerns are examples of factors that may drive regulators to consider certain design choices at certain times over others. High system-wide penetrations of PV, for example, may create certain grid management issues (e.g., duck curve) $)^{25}$ that could motivate the utilization of more granular, cost-reflective rates (e.g., TOU tariffs) or alternatives to NEM to better align customer and grid system needs. On the other hand, jurisdictions that have financially insolvent utilities or significant cross-subsidy schemes in place may be more concerned with ensuring utility revenue sufficiency and avoiding cost-shifting and may wish to encourage the use of customer-sited storage deployment primarily for self-supply or backup during grid outages.

At a high level, changes in compensation mechanism design consequently drive customer behavior and may incentivize greater behind-the-meter storage deployment, depending on the specific approach taken. Early-stage DER markets may take different approaches to compensation mechanism design than mature DER markets depending on these context-specific factors and needs. In an era of rapidly shifting technology costs, customer demand, and power system dynamics, the leapfrogging of traditional compensation mechanism design transition pathways forged by mature DER markets is entirely possible, if not likely.

\footnotetext{
${ }^{24}$ For the purposes of this report, sell rate design refers specifically to the design of a volumetric energy export rate (i.e., stated in $\$ / \mathrm{kWh}$ ) under a compensation mechanism framework designed for retail customers that is approved by regulators. In the future, this term could also be inclusive of compensation rates offered by aggregators and other third parties in the free market (i.e., not set by regulators) for grid services provided by customer-sited DER.

${ }^{25}$ A 'duck curve' refers to a phenomenon in power systems with high penetrations of coincident solar energy wherein demand during the day is depressed due to DPV production, potentially leading to steeper ramping requirements for flexible generators in the evenings when solar PV systems begin reducing output (Denholm et al. 2015).
} 


\subsection{Metering and Billing Arrangements}

This section focuses on the two primary metering and billing arrangements for behind-the-meter resources that allow for self-consumption of on-site electricity production: NEM and net billing. ${ }^{26}$

\subsubsection{Review of Net Energy Metering and Net Billing}

NEM, often referred to as net metering, is the most common compensation mechanism for DG in the United States. In NEM schemes, whenever a customer's distributed generation exceeds their consumption, their excess generation is injected to the grid for a credit in $\mathrm{kWh}$. These credits can then be used to offset their consumption from the grid in the current billing cycle and often in future billing cycles, depending on the design of the specific NEM program. Thus, it is the customer's net consumption of energy from the grid, or the consumption from the grid minus the exports from the customer's distributed generation system, which is billed. This net consumption is typically measured using a bidirectional 'net meter' meter (see Figure 8). Gross metering, with separate consumption and generation meters, can also provide the same compensation as net metering, provided the sell rate for compensation of grid injections matches the volumetric portion of the retail electricity tariff, and the crediting terms ${ }^{27}$ are sufficiently similar (Zinaman et al. 2017).

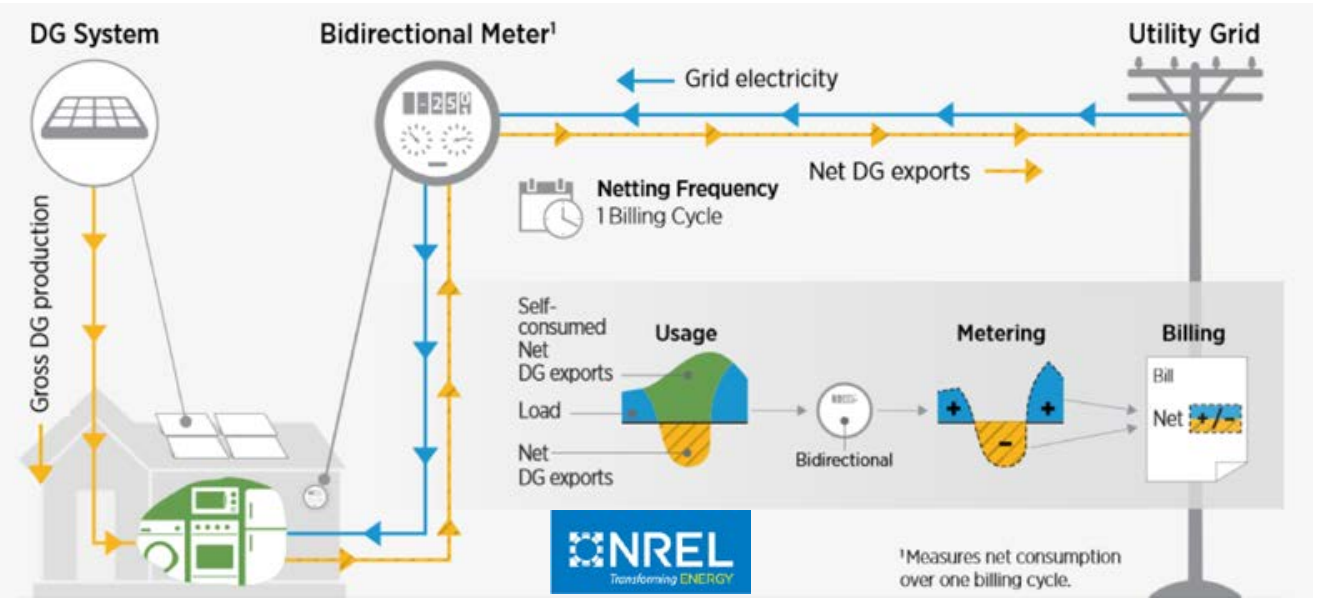

Figure 7. NEM schematic for DG system without BESS

Source: Adapted from Zinaman et al. 2017

Despite NEM's popularity in the United States, some jurisdictions have begun transitioning to net billing schemes. Under net billing, excess DG injected to the grid cannot be used to offset consumption on a kWh-for$\mathrm{kWh}$ basis in later periods (as occurs under NEM), and instead is turned into bill credit. Any DG injected into the grid is credited at a predetermined sell rate (typically less than the volumetric retail energy tariff ${ }^{28}$ ). All injections to the grid are metered in real time by an electricity export meter. Any energy consumption from the grid (which, in practice, is the customer's real-time energy demand in excess of their real-time DG production) is charged to the customer at the predetermined volumetric retail energy tariff, as usual, using a regular electricity consumption meter. At the end of the billing cycle, the customer is charged for the difference between the generation credits and the consumption debits (see Figure 9) (Zinaman et al. 2017).

\footnotetext{
${ }^{26}$ Buy All, Sell All metering and billing arrangements are not discussed, as these are effectively front-of-the-meter schemes for retail customers.

${ }^{27}$ Crediting terms define whether compensation is granted as bill credit (either in currency or kilowatt-hours) or directly as cash payments. They also determine the extent to which credit can be carried over between billing cycles and the circumstances under which credits might expire and cash payments are paid to the DG system owner. For more information, see Zinaman et al. (2017).

${ }^{28}$ Sell rates for injections at levels above the volumetric energy charge of the retail tariff also occur in some places, but typically under buy-all sell-all or nonuser DPV schemes (IEA 2019b).
} 


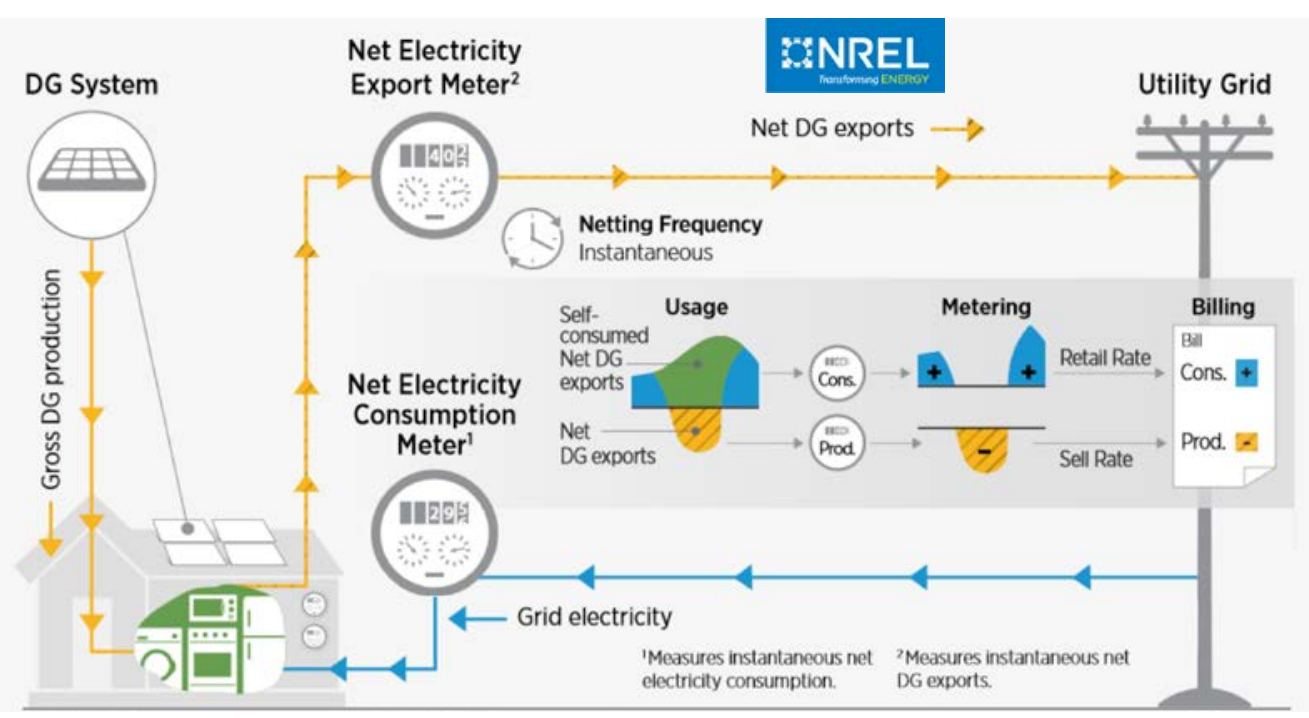

Figure 8. Net billing schematic for DG system without BESS

Source: Adapted from: Zinaman et al. 2017

\subsubsection{Implications for DPV-Plus-Storage Deployment}

NEM schemes essentially enable the utility grid to offer a form of financial storage for behind-the-meter DG systems. Whether it supports combined DPV-plus-storage systems depends on how regulations are structured.

- If the DPV-plus-storage customer is under an NEM scheme and is subject to a tariff structure that features a time-invariant volumetric energy rate, then from the customer's perspective, in financial terms, the utility distribution system serves the same function as the storage system - to bank DPV energy for that individual customer's benefit and use it at a later time. Thus, such a simple retail tariff structure under NEM does not incentivize the pairing of DPV systems with behind-the-meter storage. See Section 4.2.1.1 for a deeper discussion.

- TOU tariffs could be optimized to encourage DPV-plus-storage systems configured to export during higher value times (e.g., peak periods) NEM schemes under TOU energy rates - where distinctly valued kilowatthour credits are granted for exports based on the time of export - can help customers reduce bills and enhance the business case for DPV-plus-storage deployment. These are described more fully in Section 4.2.1.2.

Net billing schemes typically feature a sell rate for exports below the retail volumetric energy rate. As a result, customers have an economic incentive to maximize self-consumption of their DPV production, because avoiding a retail kilowatt-hour purchase from the grid through self-consumption is more valuable to the customer than receiving compensation for exports. But much of a customer's demand is typically noncoincident with times of DPV production; thus, the presence of a paired energy storage system for customers under net billing can augment the ability of the customer to self-consume their DPV kilowatt-hours, as DPV kilowatt-hours that would otherwise be exported can be stored and consumed at a later time, after accounting for roundtrip losses.

\subsection{Retail Tariff Design}

Because energy storage systems can enhance the ability of the customer to increase DPV self-consumption and avoid retail electricity purchases, the retail tariff structure tends to have a more significant effect on overall DPVplus-storage customer economics than choosing between an NEM or net billing scheme (McLaren et al. 2019).

Retail tariff design is an essential element of compensation mechanisms for DPV-plus-storage systems. From a customer's standpoint, the bill savings and credits yielded from behind-the-meter systems are intrinsically linked to retail tariff design and, thus, retail tariffs strongly influence both customer economics and deployment. From a utility's perspective, retail tariff design can promote utility revenue sufficiency and help align customer production and consumption with the needs of the larger power system. 
Importantly, many of the retail rate design strategies discussed in the following subsections that are intended to support DPV-plus-storage deployment involve the introduction of greater complexity to retail tariff structures. This is predominantly implemented through time-variant rates, as well as demand-based charges, which are intended to better reflect the real-time cost of electricity delivery. ${ }^{29}$

More complex tariff structures may require more advanced metering solutions. In practice, these advanced tariff structures have been enabled by the emergence of advanced metering infrastructure, which is able to measure and collect more granular data on electricity production and consumption. Smart infrastructure, which intelligently operate behind-the-meter energy assets and manage energy consumption - such as battery control systems, home energy management systems, and smart thermostats - can also play a large role in enabling DPV-plus-storage customers facing more complex retail tariff structures to realize bill savings. These technologies can enable customers to increase their bill savings by shifting consumption from the grid to lower price periods (in response to time variant rates) or reducing peak demand (in response to demand charges).

As utilities and regulators consider these strategies, some key practical considerations to keep in mind include ${ }^{30}$ :

- Simplicity -Will the customer be able to understand how they are being charged for electricity under the new tariff? Will the utility be administratively and technologically prepared to implement the new tariff?

- Responsiveness - Does the customer have both the understanding and the ability to adjust their energy behavior to lower their bill under the new tariff?

- Stability - Is the customer (and utility) sufficiently shielded from unreasonably high fluctuations in bill costs (and bill collections) between billing cycles?

At a high level, many regulators and utilities are attempting to balance the above considerations with a desire to, among others:

1. better align customer operational behavior with the technical requirements of the power system

2. promote fair utility cost recovery

3. reduce cross-subsidization effects between participating and nonparticipating customers

4. promote the deployment of specific technologies to meet other policy objectives.

The subsequent subsections introduce various tariff structure elements and outline considerations for implementing them in the context of DPV-plus-storage deployment. ${ }^{31}$

\subsubsection{Volumetric Energy Rates}

Volumetric energy rates are a standard tariff component designed to charge customers on a per kilowatt-hour (i.e., $\$ / \mathrm{kWh}$ ) basis for their electricity consumption. Essentially all retail electricity customers pay some form of volumetric energy charge, which often comprise the majority of customers' bills for residential customers and

\footnotetext{
${ }^{29}$ Until recently, almost all residential customers and many smaller commercial customers around the world have been subject to tariffs comprising a simple, flat volumetric electricity rate that ensured steady revenues to utilities and stable, easily understood bills for customers. But with the advent of new metering and information and communication technologies, it is increasingly possible to institute more complex tariff elements normally reserved for large commercial and industrial customers, such as time-of-use rates and demand charges. These new tariff structures will be discussed in subsequent subsections.

${ }^{30}$ These considerations are not unique to DPV and storage-plus-DPV systems, but instead apply to all retail customers subject to tariff changes; however, the presence of a storage system presents new opportunities for customers to use battery control software to be more responsive to more complex tariffs.

${ }^{31}$ Fixed charges and minimum bills - which are often used to recover fixed utility costs from retail customers-are not discussed in this section. For more information on the merits of fixed charges for DG customers, see Linvill, Shenot, and Lazar (2013). For more information on minimum bills, which have grown in prominence to address utility revenue sufficiency issues caused by DPV in the United States, see Lazar (2014).
} 
may also be true for commercial and industrial ratepayers as well. ${ }^{32}$ In the context of DPV-plus-storage systems, the following subsections will discuss:

- Time-invariant energy rates (Section 4.2.1.1)

- Time-of-use energy rates (Section 4.2.1.2)

- Nonbypassable energy rates (Section 4.2.1.3)

- Inclining and declining block energy rates (Section 4.2.1.4)

- Critical peak pricing (Section 4.2.1.5)

- $\quad$ Real-time pricing (Section 4.2.1.6)

Importantly, these rate structures are by no means mutually exclusive, and it is possible to combine elements of these rate designs when developing retail tariff structures.

\subsubsection{Time-Invariant Energy Rates}

Many residential and commercial customers are on tariffs featuring a time-invariant (often referred to as "flat") volumetric energy rate; it is the most common configuration in the United States today. These rates are deemed time-invariant because they do not change based on the time that the customer consumes energy. Time-invariant volumetric energy rates are generally simple and easy to understand for consumers, easy to implement by utilities using simple electromechanical meters, and help to protect consumers from volatility in real-time electricity prices.

Placing a DPV-plus-storage customer on a time-invariant rate eliminates the possibility of arbitrage activities, as the customer only experiences a single price for energy throughout the day. For customers on time-invariant volumetric energy rates, however, there is no connection between their load consumption patterns, their DPVplus-storage generation, and the actual system conditions at the time of consumption or generation. Absent a scenario where the utility is directly controlling the behind-the-meter system, there is no economic signal available to influence the behavior of customers to contribute to power system operations. From the utility and societal perspectives, this lack of an economic signal may constitute a foregone opportunity for the utility or utility's system to derive value from these resources.

\subsubsection{Time-of-Use Rates}

An increasing number of utilities are adopting TOU rates, driven by desires for more cost-reflective and systemfriendly pricing, renewable energy grid integration challenges, and the growing ubiquity of advanced metering technologies enabling their implementation. Some U.S. jurisdictions are adopting TOU rates. Under TOU rates, a set of predetermined volumetric energy rates are applied to a customer's consumption, where the rates change based on the time of day, week, or year their energy is consumed (Lowder et al. 2017). TOU rates vary throughout a given time period in anticipation of expected system conditions and associated time-variant costs of power system operation. The time periods and rates are set well in advance of the time of consumption and are not adjusted to reflect actual conditions on the distribution system (Hogan 2014). ${ }^{33}$ The implementation of TOU rates within billing cycles (as opposed to seasonal rates) requires metering solutions that can track energy consumption at the appropriate interval (e.g., hourly).

During periods of peak system-wide or localized demand, the utility's real-time cost of delivering electricity can increase drastically. With or without storage systems, if retail customers are even partially exposed to these price signals through a TOU rate, and if they have the ability to understand and respond to these price signals, ${ }^{34}$ they

\footnotetext{
${ }^{32}$ Commercial and industrial customers may also have demand-based charges in their utility tariffs, which, in some locations, have been increasing to be equal to or more than the energy component. The details are very location-specific.

${ }^{33}$ This is distinct from critical peak pricing (CPP) tariffs under which customers pay significantly higher rates during a set number of periods when the grid is most constrained (see Section 4.2.1.5).

${ }^{34}$ To respond to TOU rates, customers must either manually manage their electricity consumption and the operation of their storage-plus-DPV system or utilize smart infrastructure. A study on the effect of smart infrastructure in Baltimore showed
} 
may choose to reduce their consumption and thus contribute to easing system stress. ${ }^{35}$ Importantly, TOU rates tend to economically incentivize DPV-plus-storage systems relative to time-invariant rates by offering customers the opportunity to derive value from temporal differences in the retail volumetric energy rate and/or sell rate for electricity - these energy arbitrage activities may or may not be desired by policymakers and regulators. In any case, TOU rates may be a useful tool for incentivizing DPV-plus-storage system deployment.

The details of how TOU rates are designed will influence the economic and technical impact that DPV-plusstorage systems will have on customers and the utility system. Relevant TOU rate design elements include, inter alia:

- Pricing of TOU Periods - the difference in the price of electricity between the various periods (e.g., between peak and off-peak energy rates) influences the potential for customer bill savings and the incentives for customers to shift consumption from the grid to lower-priced periods. The magnitude of customer responses to TOU rates is strongly related to the peak-to-off-peak price ratio: one study suggests that for every $10 \%$ increase in this price ratio, customers in TOU pilots in the United States reduced their on-peak demand by an average of $6.5 \%$ (Faruqui 2018).

- Timing of TOU Periods - setting peak periods outside the range of DPV production hours can influence the relative economics of a storage-plus DPV system, as customers with grid-tied DPV systems would otherwise be unable to take advantage of the difference between period prices (see the "Timing of TOU Periods" text box for more details).

- Duration of TOU Periods - creating longer duration peak period windows can be less effective in incentivizing customer behavior to match the system's needs. Longer duration peaks, around 5-8 hours, require more significant behavioral changes or longer-duration storage systems, which are typically more expensive, to reduce demand for the entirety of the peak period. Shorter duration peak periods, around 2-3 hours, will require less significant behavioral changes and less expensive, lower-duration storage systems to take advantage of the period pricing differences. As these periods are set well in advance, shorter peak periods are also less likely to coincide with actual individual system peak events, potentially eliminating the system benefit of modifying customer behavior. Importantly, customers who face demand charges can still benefit from the addition of storage, even if storage system does not have enough duration to cover the entire peak period window, so long as the system can reduce the customer's maximum consumption during the peak period window.

that technology that alerted customers to prices and gave them the ability to reduce consumption during peak hours led to more significant peak consumption reductions than a TOU rate alone (Faruqui et al. 2012).

${ }^{35}$ TOU rates can be understood as a measured step in the direction of exposing retail customers to the real-time cost of electricity delivery, sending price signals to conserve energy during typical times of higher demand (or to use more energy during times of excess production), while still protecting customers from the full price volatility of real-time energy market conditions (Lowder et al. 2017). 


\section{Timing of TOU Periods}

One key element of TOU rates that can influence the deployment of storage-plus-DPV systems is the timing of the peak period. If a DPV system's generation profile occurs primarily during off-peak price hours, the system owner may experience smaller reductions in their energy charges, particularly if they also cannot shift consumption to offpeak periods. They may also be incentivized to orient their panels in a particular direction to maximize production during peak periods (e.g., orienting panels to the west to maximize later-afternoon production). A storage-plus-DPV system, however, can help customers shift consumption to off-peak periods or shift exports to the grid during onpeak prices hours.

In this way, TOU rates can improve the economics of storage-plus-DPV systems relative to grid-tied DPV systems. McLaren et al (2019) demonstrates this effect in their modeling of storage-plus-DPV for commercial customers. They found that the presence of TOU elements in the retail rate increase the economic viability of storage-plusDPV.

As PV penetration increases on power systems, previously on-peak hours may become periods of low demand and may eventually receive lower remuneration, further increasing the incentive for export-shifting capabilities. This has already happened in California, where all three of California's major investor-owned utilities have adjusted the peak period of their TOU rate to later in the evening to better align with the timing of their net load peak (Diau 2017).

\section{Jurisdiction Examples}

California-In California, all commercial, industrial, and agricultural customers are required to be on TOU rates. California utilities have also begun transitioning residential customers away from a flat rate toward a residential TOU rate. These residential TOU rates have already gone into effect for San Diego Gas \& Electric and will go into effect in 2020 for Pacific Gas \& Electric and Southern California Edison (SCE). Any customer who applies for California's Net Energy Metering 2.0 incentive scheme will be required to join a TOU rate. SCE offers a TOU rate (known as "TOU-D-PRIME") specific to households with electric vehicles, behind-the-meter storage, or heat pumps (SCE 2019). This rate has a seasonal and weekly aspect with different price periods depending on whether consumption occurs during the summer or winter, on weekends or weekdays. Regardless of season or day of week, the peak period occurs in a 5-hour window from 4 p.m.-9 p.m., well outside optimal solar generation hours, yet during SCE's anticipated system peaks.

\subsubsection{Nonbypassable Volumetric Charges}

A nonbypassable volumetric charge is a volumetric energy rate that a customer pays on every kilowatt-hour of electricity they consume from the grid (i.e., their grid consumption); however, the charge cannot be offset by receiving credits for DPV-plus-storage exports. In practice, this means that when the customer purchases electricity from the grid, they would be billed at the applicable volumetric energy rate and the nonbypassable charge rate. But when the customer exports DPV-plus-storage electricity, they are only compensated at the volumetric energy rate. ${ }^{36,37}$ During the billing process, nonbypassable charges and volumetric energy rates would be tabulated separately. These charges reduce bill savings for DPV-plus-storage customers and are primarily implemented to promote utility fixed cost recovery, although they can support a wide range of functions, including energy efficiency or clean energy incentive programs.

\section{Jurisdiction Examples}

California - Customers under California's NEM 2.0 scheme are subject to a nonbypassable volumetric charge for purchasing grid electricity that cannot be offset. In California's NEM 1.0 scheme, customers paid a similar set of tariff riders on their net energy consumption; however, under the new scheme, a nonbypassable charge is applied to gross electricity consumption, and are tabulated separately than volumetric energy charges (CPUC 2016a).

\footnotetext{
${ }^{36}$ Nonbypassable volumetric charges are a more useful construct for customers under NEM schemes, where kilowatt-hour credits are granted to offset energy charges, as opposed to net billing schemes which already feature energy charges that can be considered nonbypassable.

${ }^{37}$ The introduction of a nonbypassable volumetric charge into an NEM scheme would, based on the definitions established in this report, create a net billing scheme.
} 
Using the definitions established in this report, the introduction of a volumetric nonbypassable charge to California's NEM program effectively makes it a net billing scheme.

Israel-Under Israel's NEM scheme, NEM customers are subject to a nonbypassable volumetric charge for both gross grid electricity consumption and DPV self-consumption. The charge was explicitly structured to recover utility costs from customers who depend on the bulk power system during periods of time without solar production. The Israeli NEM framework also features an additional nonbypassable volumetric charge for all DPV exports that is intended to remunerate the utility for the cost of integrating these variable renewable energy resources into the power system, following a calculation methodology established by the International Energy Agency (PUA 2014).

\subsubsection{Inclining and Declining Block Charges}

Under inclining block charges (IBCs) and declining block charges (DBCs), the volumetric rate for electricity increases or decreases, respectively, as cumulative electricity consumption within the billing cycle increases (see Figure 10).
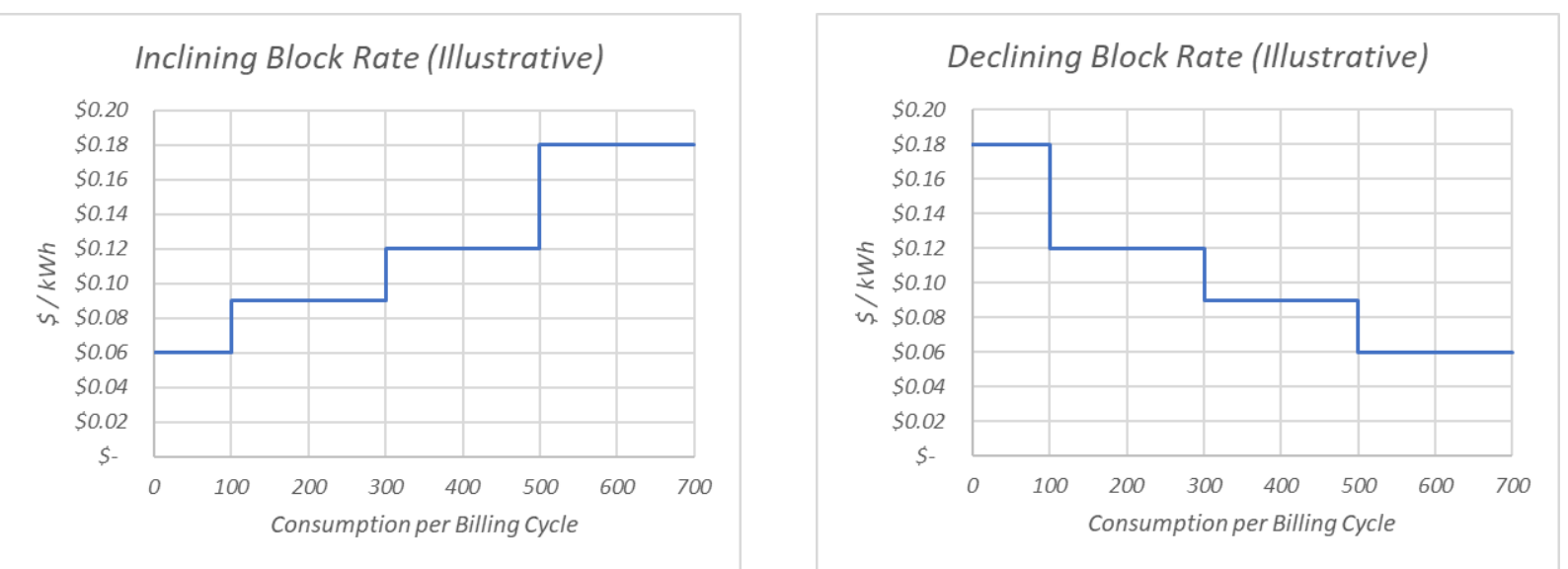

Figure 9. Illustrative diagram of inclining block rate (left) and declining block rate (right)

Source: Original

IBCs are common retail tariff components already present in tariff structures in many countries. They are not typically implemented specifically for DPV or DPV-plus-storage customers but can be considered as DPV or DPV-plus-storage programs are being designed. IBCs are typically implemented to promote energy conservation, as increasing energy consumption leads to higher retail prices, or as a matter of social policy, as policymakers may wish to subsidize low-usage (and often lower income) customers through tariff structures. For DPV customers, IBCs encourage DPV self-consumption, and could further incentivize the pairing of DPV and storage systems if consumption blocks grow sufficiently more expensive relative to the sell rate for exported DPV production.

DBCs, while relatively uncommon in the United States, can be considered as a tool to promote utility cost recovery from classes of customers which are increasingly supplying their own electricity through grid-tied DPV and DPV-plus-storage.

\subsubsection{Critical Peak Pricing}

An additional time-variant rate for regulators to consider is CPP. Under CPP schemes, prices are generally set to significantly higher levels during a set number of periods of system stress and will be lower outside of those periods. These higher peak prices are designed to better reflect the actual energy and capacity costs associated with providing electricity service during times of system stress.

Importantly, CPP schemes are activated in response to system needs by utilities. Utilities are required to notify customers at least one day in advance of an expected critical peak event, usually through phone calls or text 
messages. Regulators will typically limit the number of critical peak periods a utility can declare (e.g., 15 days per year during the season of system peak). Critical peak events can either be during the same set of hours for all events, similar to TOU on-peak rates that always occur during a predetermined set of hours, or can change from event to event as the system needs change. CPP schemes tend to provide a stronger price signal than more conventional TOU rates, while exposing customers to higher energy prices during relatively fewer number of hours per year. In some jurisdictions there has been resistance to implementing CPP because of the significantly higher prices during critical peak events. Customers may also consider CPP tariffs to be more intrusive as they must be regularly contacted by utilities in advance of the event (Faruqui et al. 2012). ${ }^{38}$

As with more conventional TOU rates, regulators must balance multiple objectives when determining the price differential between critical peak events and normal system operating periods. Larger differences between the two sets of periods tend to produce stronger demand reductions in customers and thus better align customer behavior with system needs. One variant of CPP is variable peak pricing, in which the price during critical peak events can change from event to event to better reflect system conditions. In these cases, the new price is typically relayed to the customer when they are informed of the critical peak event. The ability of the customer to understand and respond to CPP notifications is key. The timing of the critical peak events also influences the economics of gridtied DPV and DPV-plus-storage systems. As with TOU rates, critical peak events that fall outside of normal solar generation hours will create an incentive to pair grid-tied DPV systems with storage systems. Importantly, CPP can be combined with TOU rates, such that the utility is allowed to increase the normal on-peak pricing during critical peak events to further stimulate load reductions when the system is stressed.

\section{Jurisdiction Examples}

California-As a part of a pilot project, Sacramento Municipal Utility District (SMUD) outfitted 34 net-zeroenergy smart homes with grid-interactive DPV-plus-storage systems to test their ability to provide a wide range of services such as regulation, spinning reserve, demand response, and peak load shifting (Jimenez 2014). In addition to the DPV-plus-storage, the houses were installed with smart thermostats to help enable customer responses to changes in electricity prices. Approximately half the customers were placed on a combined TOU$\mathrm{CPP}$ rate to test the new rate's ability to induce load reductions. Customers on average reduced their demand by $1.35 \mathrm{~kW}$ during peak periods and by $2.66 \mathrm{~kW}$ during critical peak events (Trabish 2015).

\subsubsection{Real-Time Pricing}

Real-time pricing, in which customers see and pay for the actual price of electricity at any time period of the day, ${ }^{39}$ has only seen limited deployment for smaller commercial and residential classes, due to their historical inability to respond to such dynamic rates. For customers with sufficient infrastructure to see and react to realtime prices, these tariffs allow for the best alignment between customer consumption and utility electric system requirements (Hogan 2014). The increased price volatility associated with real time pricing may provide a strong incentive to store cheaper energy during off-peak periods for self-consumption during on-peak periods, which could increase the appeal of DPV-plus-storage; however, there is little experience and limited research to date on real-time pricing for residential and commercial customers and its effect on DPV-plus-storage economics and deployment.

\section{Jurisdiction Examples}

Texas-In Texas, which features a competitive retail energy market, some energy retailers offer "indexed" energy pricing schemes that shift a portion of wholesale energy market price risk to customers by tying changes in various market indices to retail energy prices (Texas Power Guide 2017).

\footnotetext{
${ }^{38}$ Customer concerns surrounding privacy and intrusiveness under CPP tariffs can be partially alleviated by allowing the customer to designate which method of communication they would prefer (e.g., email, text, phone call). The use of enabling technologies, such as smart thermostats and water heaters, that respond automatically to utility price signals could also be used to minimize these concerns (Faruqui et al. 2012).

${ }^{39}$ In jurisdictions with established wholesale electricity markets, customers are generally able to observe the wholesale price of electricity. In jurisdictions with vertically integrated utilities, customers' prices could be determined by the utility or regulator based on an ex-post evaluation of real-time system conditions.
} 
Illinois-Ameren Illinois and ComEd offer real time pricing tariffs for residential customers. Under Ameren's Power Smart Pricing tariff, hourly prices for the next day are set the night before and communicated to customers so that they can adjust their consumption (Ameren Illinois 2019). Under ComEd's Residential Real Time Pricing tariff, prices are based on the actual hourly market price of electricity during the day, and customers on the tariff are alerted when these prices are high or are expected to be high so that they can adjust consumption (ComEd 2019). Both rates include an additional charge to cover the cost of ensuring adequate capacity, based on their contribution to system peak demand, similar to a demand charge. Again, the impact these tariffs have on customer adoption of DPV-plus-storage is unclear at this time.

\subsubsection{Demand Charges}

Demand charges are a per-kW fee applied to a customer's maximum consumption within a specified period. Demand charges are common for commercial and industrial customers, but less so for residential customers' however, utilities are increasingly exploring demand charges for smaller (i.e., residential and small commercial and industrial) customers and several U.S. jurisdictions have evaluated or implemented demand charges for customers with DG (Proudlove et al. 2018). Although demand charges typically only apply to demand during a single period, either during the customer's highest demand (i.e., a non-coincident demand charge) or the system's highest demand (i.e., a coincident demand charge), it is possible to create multiple demand charge periods, similar to TOU periods, in which a customer's demand charge is levied based on their peak consumption during specific times of day.

Many of the costs associated with delivering energy are closely related to the system's (and customer's) peak demand for electricity $(\mathrm{kW})$, including costs of ensuring sufficient transmission, distribution, and generating capacity, rather than the total energy consumption within a given period $(\mathrm{kWh})$. Although these costs could be, and historically have been, recovered by the utility through volumetric energy rates, the use of demand charges can send an economic signal to customers to adjust their consumption during system peak hours to reduce system costs and improve reliability. Thus, demand charges may provide better incentives to customers to engage in load management through energy efficiency, storage, or DPV by providing an opportunity for bill reductions via reducing their demand.

Key elements to consider when designing a demand charge include:

- Coincident versus noncoincident charges - A noncoincident demand charge is a per- $\mathrm{kW}$ fee that is applied to a customer's peak demand during a billing cycle. Noncoincident peak demand charges can be an effective tool for reducing individual customers' peak demand, and the generation and network costs associated with serving that peak demand. A coincident demand charge is a per-kW fee that is applied to a customer's highest demand during the system's peak demand period, the timing of which is typically specified in advance to the customer (Lazar and Gonzalez 2015). Coincident peak demand charges are an effective tool for aligning the customer's behavior with the needs of the power system by encouraging them to reduce consumption during periods of high demand (Passey et al. 2017). While both demand charge structures offer opportunities for bill reductions for DPV-plus-storage customers, coincident peak demand charges may offer a greater potential to alleviate system peak concerns relative to non-coincident charges (Darghouth et al. 2019). While noncoincident peak demand charges requires continuous monitoring and control of the customer's grid usage throughout the day to be effectively minimized, coincident peak demand periods are typically only four hours in length, making effective demand management a more feasible task.

- Peak demand period timing - the timing of the demand period (if using coincident peak demand charges) will influence the economics of DPV-plus-storage systems relative to grid-tied DPV systems. If the peak demand window falls outside of the peak production period for a DPV system, this may serve as incentive to utilize DPV-plus-storage systems.

- Peak demand period duration - longer peak demand periods may reduce the customer's ability to reduce their maximum demand for the entire duration of the demand period. Shorter peak demand periods can better allow customers to shift their consumption outside the peak demand period and reduce both their strain on the power system and their bills, either through storage or behavioral changes. Put differently, demand charge reductions are typically greater from energy storage systems when peak demand periods correspond to the duration of energy storage capacity (Darghouth et al. 2019). 
- Averaging interval - the peak customer demand applied to the per-kW demand charge fee is based on the average of their demand over a specific interval of time (e.g., every 15 minutes). Longer averaging intervals tend to create lower peak demand values used in the calculation of the demand charge, thereby reducing the overall opportunity for storage-plus- systems to lower demand charges. Shorter averaging intervals more accurately represent the customer's contribution to system peak demand and provide a greater opportunity to reduce demand charges.

Although DPV is also capable of reducing customer demand from the grid, its variable and uncertain nature and limited temporal range of generation (i.e., daytime) may interfere with its ability to reduce customer demand charges, such as if peak demand occurs after sunset or if cloud cover interrupts generation during a relevant demand measurement period (Darghouth et al. 2017). Unlike grid-tied DPV systems, DPV-plus-storage systems are - if managed properly - more dispatchable in nature. This allows these resources to more reliably reduce demand charges relative to grid-tied DPV systems, especially if combined with controls specifically designed to limit peak demand (Neubauer and Simpson 2015; Hledik and Greenstein 2016). Thus, as with TOU rates, the implementation of demand charges has the potential to increase the economic attractiveness of DPV-plus-storage systems. McLaren et al. (2019), for example, demonstrate that the presence of demand charges, particularly those greater than $\$ 10 / \mathrm{kW}$, among commercial customers, contribute to larger bill savings for DPV-plus-storage systems relative to grid-tied DPV systems (McLaren et al. 2019).

Regardless of whether the customer has a grid-tied DPV or a DPV-plus-storage system, a key factor in the ability of these systems to reduce demand charges is the customer's underlying load pattern. Customers with "peakier" load patterns, assuming the peak of their load coincides with the demand peak period, are more likely to experience greater reductions in demand charges through the use of storage systems relative to customers with flatter demand patterns (Darghouth 2019).

\section{Jurisdiction Examples}

Massachusetts-One Massachusetts investor-owned utility, Eversource, gained approval from the public utility commission to institute a residential demand charge for customers who own rooftop-PV starting in 2019, based on noncoincident peak demand (Walton 2018). The rate structure will need to be revised, however, due to recent legislation requiring any residential demand charges to be based on coincident peak demand (Commonwealth of Massachusetts 2018). At the time of this writing, it is too early to tell whether this demand charge will incent DPV-plus-storage deployment in Eversource's service territory.

Arizona-In Arizona, utilities have begun offering rates that combine TOU rates with demand charges. Arizona Public Service (APS), for example, offers a Saver Choice Plus rate and a Saver Choice Max rate (APS 2018b; 2018a). In each of these rates, a customer's bill consists of three elements: (1) a basic service charge (\$/month) that remains constant regardless of energy usage; (2) a peak hour usage (or demand) charge, which applies a flat charge $(\$ / \mathrm{kW})$ to the highest demand of electricity during the system's peak demand hour for that billing cycle; and (3) an energy charge $(\$ / \mathrm{kWh})$ that is applied to overall energy use, with a higher rate applied to energy used during predetermined on-peak periods and a lower rate applied to off-peak periods.

Jharkhand and Gujarat (India) - Although not enacted, tariff proposals to the Jharkhand and Gujarat Energy Regulatory Commissions are currently being considered which establish conditional time-of-day demand charges for certain customer classes. The proposals include the creation of 'Peak Billing Demand' (PBD) and 'Off-Peak Billing Demand' (OPBD) periods that align with current time-of-day periods in the states. The tariff is structured such that - as long as OPBD stays below the customer's contract demand ${ }^{40}$ - customers can use electricity freely during off-peak periods without a demand charge being levied. Rather, the demand charge is levied based on the PBD. This structure offers the opportunity for customers who can shift some portion of their demand from peak periods to off-peak periods to reduce their overall demand charge.

Despite their similar structure, Saver Choice Max has a significantly higher demand charge and lower energy charges than Saver Choice Plus. Customers able to shift more of their total energy consumption (kWh) during the predetermined peak hours to off-peak hours would benefit from the Saver Choice Plus plan, while those able to

\footnotetext{
${ }^{40}$ Defined as the maximum power (in $\mathrm{kW}$ ) that a customer is allowed to take from the network. In general, if contract
} demand is exceeded, customers are asked to pay a penalty. 
reduce their highest instantaneous demand consumption $(\mathrm{kW})$ during the predetermined peak hours would benefit from the Saver Choice Max plan. Under either plan, DPV-plus-storage systems could allow customers to shift their consumption from periods of high prices to periods of low prices, as well as reduce the demand charge portion of their bill. Data on the impact of these tariffs on DPV-plus-storage economics and deployment are not available at this time.

\subsection{Sell Rate Design}

The sell rate, or the level at which DPV exports to the grid are credited, can be an important factor in the economics of both grid-tied DPV and DPV-plus-storage systems. Under NEM, the sell rate is the volumetric energy rate of the retail tariff. ${ }^{41}$ In practice, under typical time-invariant volumetric rates, there is minimal economic benefit for pairing storage and DPV, as the grid is effectively being utilized as a free financial battery for DPV kilowatt-hour credits.

Under net billing, however, the sell rate is typically lower than the volumetric energy rate. Under these circumstances, there is an economic incentive to pair solar and storage together, since avoiding consumption from the grid through self-consumption is more valuable than exporting DPV generation. As with volumetric retail rates, the sell rate can be dynamic and time-variant in order to incentivize exporting of energy to the grid during certain peak periods or to discourage exports during periods of low demand.

With proper metering infrastructure in place, it may also be possible to implement distinct sell rates for DPVoriginated energy versus grid-originated energy for DPV-plus-storage systems. While this possibility may not be immediately relevant for most programs, implementing distinct sell rates may help to encourage more fair and appropriate remuneration for customers and enable participation in aggregation schemes.

\section{Jurisdiction Examples}

Hawaii-Given Hawaii's extensive penetration of DPV resources, the region is coping with large injections of solar energy and distribution system limitations during the day and high ramping requirements for dispatchable generators in the transition from day to evening as solar resources taper off. Encouraging some customers to store this energy on-site and either consume this energy or send it back to the grid in the transition period or evening peak period not only helps reduce the surplus injections and address distribution limitations during the day, but also to mitigate the aforementioned higher ramp rate requirements. In this context, a series of tariffs was rolled out in 2015 to replace Hawaii's legacy NEM program. These tariffs were designed to limit the amount and time at which energy could be exported to the grid from customers. Under one of these, the 'Smart Export' tariff, customers with DPV-plus-storage installations are only compensated under a net billing scheme for energy exported to the grid between 4 p.m. and 9 a.m., while receiving no credits during the hours of prime DPV production capability (HECO 2019b). By creating a time-variant sell rate, participating customers are incentivized to store their excess DPV energy during the day and export it in the transition and evening periods.

In summary, the design of compensation mechanisms offers a number of opportunities to meet program goals. Metering and billing arrangements can emphasize different objectives and can be structured to support the highest regulatory priorities. Retail tariff design has a variety of volumetric energy rate options that can promote a variety of market outcomes, and demand charges are becoming an increasingly important component for reducing both customer bills and utility costs, particularly for DPV-plus-storage facilities. Finally, sell rate design can be an important component for decisionmakers to influence how customer's self-generation is utilized. In the future, separate compensation mechanisms for the provision of grid services may be an important economic factor for DPV-plus-storage customers.

${ }^{41}$ There may be a separate sell rate for expired $\mathrm{kWh}$ credits. 


\section{Step III: Design Metering and Technical Configuration Requirements}

Key Insight for Indian Stakeholders
This section focuses on designing requirements to ensure both that interconnecting systems have adequate
metering to track the flows of energy necessary to implement compensation mechanisms, as well as adequate
measures in place to prevent undesirable flows of energy. As well, the section discusses how the addition of
storage can greatly complicate the flows of energy possible, and outlines how regulators in other jurisdictions
have managed this complexity to ensure continued progress on achieving their objectives.
In India, most existing regulations do not explicitly address the interaction of storage with the power system,
nor do they explicitly state which power flows in DPV-plus-storage systems should be measured or prohibited.
This means that in India, metering and grid requirements may need to be revisited, especially for more grid-
interactive applications of DPV-plus-storage (see Section 5.1). The FOR Draft Model Regulation for Grid
Interactive Distributed Renewable Energy Sources document does not explicitly take a position on whether
behind-the-meter storage systems are allowed to discharge to the grid during normal operating conditions
(FOR 2019). In jurisdictions that want to take advantage of the various services that batteries can provide,
metering and grid requirements will likely need to be updated to allow for discharging power to the grid.
Metering and grid requirements all have an associated cost, and SERCs can carefully weigh these costs against
the potential of a given system to interfere with normal grid operations or to meaningfully affect compensation
mechanism integrity (see Section 5.3). Differentiating metering and grid requirements based on customer
class, voltage interconnection level, or system size may help minimize undue cost burdens for customers, as
well as administrative costs for utilities that must review and approve these systems.

The prioritized regulatory objectives identified, as well as the subsequent design of compensation mechanisms, tend to be a significant driver of technical configuration requirements and metering requirements for DPV-plusstorage systems.

Technical configuration requirements are the rules governing the allowed flows of energy between the customer's load panel, ${ }^{42}$ the DPV system, the storage system, and the utility distribution system. Importantly, the responsibility for determining the exact technical details for how storage and DPV system are electrically coupled behind a customer's meter is typically left to the installer, as well as relevant codes and standards (in particular, the National Electric Code [NEC] in the United States). Utilities, on the other hand, typically have comparatively little input into the configuration of these DERs behind the service meter. Regulators may be concerned with ensuring there is both clarity and certainty on the allowed technical configurations specified within relevant technical standards, codes and interconnection rules, and that no undue cost burdens are being placed on either participating or nonparticipating customers. Furthermore, regulators and utilities may be concerned with restricting particular power flows (e.g., battery exports to the grid) to accomplish certain objectives.

Metering requirements can be defined as rules governing which exact flows of energy must be measured using metering equipment, the specific technical requirements of metering infrastructure that must be installed, and where each meter must be placed within known technical configurations to ensure all desired energy flows are accurately measured. Compensation mechanisms designed to meet specific sets of prioritized objectives directly influence metering requirements. For instance, if DPV-plus-storage customers are placed on a TOU tariff, then the metering requirements must specify the ability of the meter to measure electricity consumption for prespecified time windows, which may necessitate the use of advanced metering infrastructure. Similarly, utility requirements may extend to metering infrastructure that is behind the service meter (which is the typical jurisdictional line of

\footnotetext{
42 The load panel or service panel is the point of entry for power from the grid into the customer's premise. The service panel will be situated on the user side of the utility service meter and in nearly all circumstances belongs to and is the responsibility of the customer, not the utility.
} 
demarcation for utilities) to track certain power flows. Regulators must balance a desire to avoid excessive metering costs for customers and consumer privacy concerns with the need to encourage accurate measurements and billing practices through adequate metering infrastructure.

Both the technical configuration and the metering requirements imposed by regulators have important implications for the services battery systems can provide and the economics of these systems to customers, as well as the integrity of compensation mechanisms (see Section 5.3).

\subsection{Technical Configuration Requirements}

Energy storage systems can be connected (or coupled) to a DPV installation on either the AC or DC side of an inverter. The decision to couple DPV and storage systems in AC or DC configurations is made by either the customer or installer and depends on various factors, including the size of the systems, the desired use cases, and whether the system is new or retrofitted. Most new, smaller DPV-plus-storage installations are designed and built as DC-coupled systems, whereas retrofits with existing DPV systems or larger systems tend to be AC-coupled. For systems that primarily use DPV energy to charge the battery for use at a later time, AC-coupled systems have higher efficiency losses (due to multiple AC-DC conversions) than DC-coupled systems (Ardani et al. 2017). DCcoupled systems can be less expensive as they require only one battery-based inverter, whereas AC-coupled systems require a battery-based inverter and a grid-tied inverter for the PV array (Mullendore and Milford 2015).

Both AC- and DC-coupled configurations can be allowed by regulators and utilities as each system design suits different customer classes and use cases. Rather than exclude a particular coupling category, regulators may choose to allow a subset of the possible configurations for each coupling category to ensure that regardless of whether the system is AC- or DC-coupled, it engages only in desired operational behavior. In other words, it may be more pragmatic for regulators to be concerned with the allowed flows of energy between the customer's load panel, the DPV system, the storage system, and the utility network, rather than whether a particular system is ACor DC-coupled. ${ }^{43}$ Unlike other forms of generation, storage has the capability to charge from either a paired DG resource or directly from the grid, and, absent specific discharge controls, it can export both grid-sourced and DGsourced energy to the grid, as well.

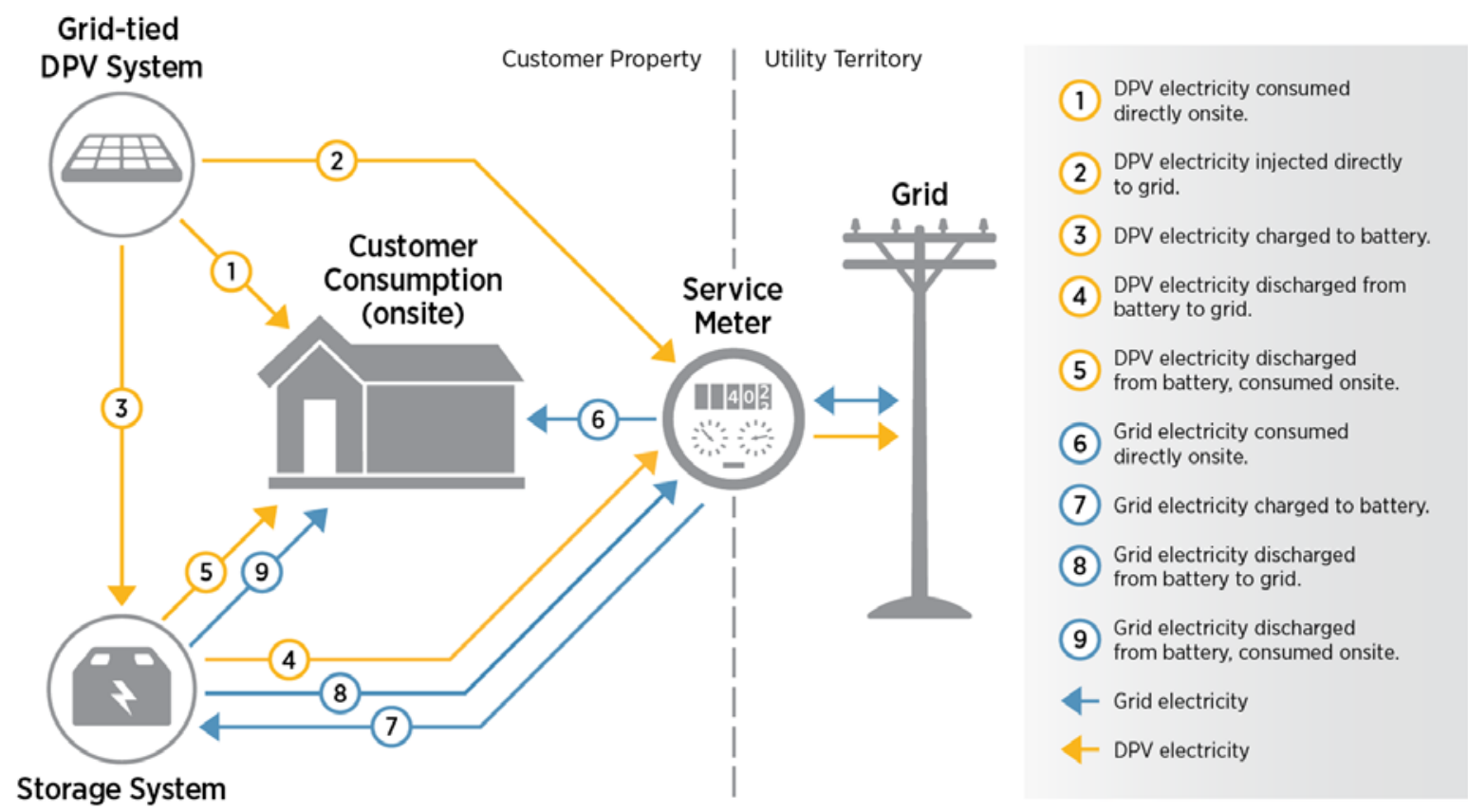

Figure 10. Energy origins, pathways, and destinations for behind-the-meter solar-plus-DPV customers Source: Original [Illustration by Christopher Schwing, NREL]

\footnotetext{
${ }^{43}$ For more technical details on common technical configurations, see Appendix D.
} 
Note: The "Service Meter" icon is intended to serve as a jurisdictional delineation between the customer and the utility. This illustrative diagram of potential energy flows does not depict other meters that may be necessary or appropriate for implementing a particular compensation mechanism.

Figure 10 offers a conceptual diagram of the various origins, pathways and destinations of electricity for a DPVplus-storage customer. Some regulators may wish to promulgate technical configuration requirements that constrain certain pathways of electricity flows (e.g., limiting the ability of the storage system to charge ( (7) ${ }^{44}$ ) from or discharge ( (4) or (8) ) to the electricity system for safety or other reasons). One common issue that regulators may be concerned with is mitigating inadvertent exports, in which non-exporting systems send energy back to the grid due to unanticipated mismatches between customer load and battery discharging, creating power quality disturbances or operational concerns for line workers during emergencies that cause local or larger area outages. Another concern may be cases in which both the DPV and battery system export their maximum capacity to the grid and potentially overload utility and/or customer equipment. Both issues can be mitigated through the implementation of battery control system requirements, which can be stipulated independently by or in collaboration among regulators, utilities, and other authority having jurisdictions (examples are included in Horowitz et al. 2019; Baldassari 2018). Battery control system requirements determine the allowable operations a battery system can engage in and can have important implications for the economics of the DPV-plus-storage system for customers, which may prompt regulators to take action to balance utility concerns with customer/installer interests. Related regulatory issues and approaches will be discussed in Section 5.3

\subsection{Metering Requirements}

Metering requirements will vary by the type of design configuration and whether a system is a retrofit or new installation, and will also be strongly influenced by the compensation mechanism that the DPV-plus-storage system is subject to. At a high level, there are a variety of electricity flows that may be relevant for metering (see Figure 11), depending on the intended use case of the DPV-plus-storage system, such as total DPV exports ( (2) + (4) ) to validate export credits, DPV production to validate renewable energy production ( (1) + (2) + (3) ), gross grid consumption ( (6) + (7) ) to accurately bill a nonbypassable volumetric charge, or total grid exports ( (2) + (4) +8 ) for use in a virtual power plant scheme, among other quantities.

According to McAllister et al. (2019) "directly metering behind-the-meter storage systems can give a utility additional insight into how storage systems are operating." Nevada's interconnection requirements, for example, require that storage systems be deployed with separate utility meters, the cost of which must be borne by customers and project developers. Separate metering provides data that can be used for distribution system planning, retail tariff design, and verification of compensation mechanism credits, according to NV Energy (McAllister et al. 2019; NV Energy 2018a). But requiring separate metering of storage systems increases costs for developers and customers, may require additional utility administrative efforts to process data, and may also raise customer privacy concerns. ${ }^{45}$ Inverter software controls may be able to provide data that can assuage utility concerns about compensation mechanism credits and arbitrage activities, and serve as an alternative to separate metering ${ }^{46}$ (Noh et al. 2018); however, there is still uncertainty as to whether inverter measurements are sufficient for tariff and policy compliance (from the utility perspective), have sufficient cybersecurity features, or cross traditional lines of demarcation between customers and utilities. Furthermore, inverter data may be output in a separate format than traditional metering data that utilities have experience processing, creating additional challenges for their implementation. In general, the presence of a DPV-plus-storage system may complicate efforts to accurately implement DG compensation mechanisms from the standpoint of metering and billing, relative to a grid-tied DPV system.

\footnotetext{
${ }^{44}$ Circled numbers (e.g., (7) ) refer to specific flows in Figure 10.

${ }^{45}$ Granular battery storage use information collected by a separate meter can potentially reveal the behavior and activities of a residence or business at a level of detail that customers may find intrusive.

${ }^{46}$ Utilizing inverter software as a utility-grade meter is an emerging opportunity being tested by some U.S. utilities. For instance, under Vermont's Green Mountain Power Resilient Home program, customers can either lease or use their own behind-the-meter storage system, receiving backup power services during outages and otherwise allowing Green Mountain Power to dispatch their system to reduce system-wide demand charges. The storage system is also utilized as a meter, effectively replacing an older single-purpose meter with a software-enabled smart meter that can measure and communicate real-time usage data to the utility.
} 


\subsection{Preserving Compensation Mechanism Integrity}

Metering and billing arrangements such as NEM or net billing are typically implemented to reward exported energy that is generated from an eligible distributed generation system (e.g., DPV). As DPV-plus-storage systems continue to permeate U.S. power markets, some decision makers have placed an increased emphasis during regulatory proceedings on ensuring that grid-supplied energy that is later exported by the storage system does not yield a financial reward using the DPV metering and billing arrangement. Addressing this issue can be broadly referred to as preserving the integrity of the DG compensation mechanism. A separate but sometimes related issue is monitoring and reducing energy arbitrage activities. Energy arbitrage is where DPV-plus-storage customers rely on time-variant price differences in the retail energy rate and profit by buying grid-supplied energy during less expensive periods and selling it back to the system during more expensive periods. ${ }^{47}$

Compensation mechanism integrity issues are typically addressed through technical configuration and metering requirements. In practice, decision makers have utilized several types of approaches, which are listed below from the simplest (and therefore least expensive for the user) to the more complex (with the highest relative costs). These include:

1. Estimating the customers' gross DPV production or DPV-originated energy exports using a simplified methodology

2. Mandating specific DPV-plus-storage technical configurations to prevent storage systems from charging from or exporting to the utility grid

3. Requiring adequate metering to track all quantities relevant to and from most or all devices for preserving compensation mechanism integrity.

Importantly, these approaches are not mutually exclusive. For instance, system size limits can be used to apply distinct approaches to DPV-plus-storage systems of different sizes within the same jurisdiction (see California example in Section 5.3.1). This is applied to ensure that the administrative and infrastructure-related costs associated with preserving compensation mechanism integrity are commensurate with the potential impact of the corresponding systems. Offering alternative options that are equally satisfactory approaches for regulators and utilities to ensure compensation mechanism integrity can help system owners choose the most appropriate method for their specific set of circumstances and intended uses.

While each of the three methods discussed in subsequent sections has associated drawbacks, these drawbacks may be applicable to or precluded by specific regulatory objectives. Tracking clean energy production for regulatory compliance for larger-scale behind-the-meter systems might fit best with a highly accurate method, such as requiring additional metering infrastructure. Preventing the inaccurate crediting of energy from ineligible sources, meanwhile, could be managed through limiting technical configurations, which are financially and administratively less burdensome than additional metering. Conversely, maximizing the value of the battery to the grid could be achieved through estimation or metering methodologies which do not limit the operational capabilities of the DPV-plus-storage system. The relative priority of each goal tends to dictate the method chosen. In general, the more granular control and observability one wants over the DPV-plus-storage system, the more expensive or administratively cumbersome the appropriate method may be to implement.

\subsubsection{Using Estimation Methods for DPV-Plus-Storage Export Crediting}

One method to ensure DPV-plus-storage system owners are fairly compensated without the need for additional metering is to set a cap on export credits available to these systems based on an estimation of expected DPV generation. At the end of each billing cycle, the customer's metered exports are compared against an estimation of generation conducted by the utility using a prespecified methodology, and the customer receives the lower of the quantities as a bill credit. In practice, these approaches can be used when the cost of additional metering

\footnotetext{
${ }^{47}$ Energy arbitrage activities conducted by retail customers are not inherently negative for power systems. In some cases, they may be a cost-effective means to reduce and/or serve peak demand, or provide other valuable load shifting services.

However, depending on how TOU rate are designed, retail arbitrage activities have the potential to exacerbate existing costshifting issues associated with DPV deployment. A discussion of the costs and benefits of retail arbitrage activities is outside the scope of this report.
} 
represents a large proportion of overall project costs (i.e., for smaller systems) or when the risk/scale of compensation mechanism integrity violations (i.e., gaming and arbitrage activities) is low.

Although not as accurate as direct metering, this simplified method gives an approximate value for generation from the eligible DPV system without the need for customers to install additional metering equipment beyond what is required for a grid-tied DPV system. Given an adequately granular estimation of the solar resource available in a specific time period and the size of the generating system in question, utilities can use technoeconomic performance analysis tools (e.g., NREL's System Advisor Model) to estimate the output of a qualifying generating system. Utilities can also use more simplified approaches, such as determining a rough $\mathrm{kWh}$ per $\mathrm{kW}$ factor for energy production for systems within a given region. The values estimated from these methods can then be used to grant the customer credit for their generation based on the size of their DPV system, regardless of the charging or discharging of their paired storage system. While estimation approaches are the least expensive approach to preserving compensation mechanism integrity, they are also likely the most inaccurate and may entail an administrative burden for utilities who must develop estimation methodologies and integrate them into bill processes.

\section{Jurisdiction Examples}

California - in California, an NEM scheme remains the primary mechanism for compensating DERs like DPV. For DPV-plus-storage systems where the battery power output is under $10 \mathrm{~kW}$, the California Public Utilities Commission (CPUC) implemented a generation credit estimation methodology in lieu of requiring more metering equipment or limiting the capability of the battery to charge/discharge from the grid. Under this scheme, California utilities are required to calculate the projected DPV generation for each DPV-plus-storage customer in a particular month in a particular region using a calculation methodology approved by CPUC (CPUC 2016b). Customers are then assigned the lesser of the actual exports to the grid or the estimated DPV generation. While this methodology tended to overestimate DPV production (unless a customer has no on-site consumption for an entire month), the ability of smaller customers to game the NEM compensation mechanism was assumed to be negligible. While the initial scheme was implemented in 2016, the methodology was updated by the regulator in 2018, following complaints from utilities that the analysis process was too administratively burdensome; currently, a simpler kWh-per-kW profile for each climate zone in the region is now used (CPUC 2018b). This profile is used along with the DPV system size to determine the likely amount of renewable energy exported to the grid, and customers are credited for exports to the grid up to this estimation. Additionally, provided the storage system is under $10 \mathrm{~kW}$, there are no limitations on the maximum output power of the battery system relative to the maximum output capacity of the paired, NEM-eligible generator. This is partly due to the limited battery sizes below $10 \mathrm{~kW}$ available to customers, and partly due to the limited ability of smaller battery systems to meaningfully violate NEM integrity principles at-scale (CPUC $2014 ; 2016 \mathrm{~b}){ }^{48}$

\subsubsection{Ensuring Accurate Metering Through Design Configuration Limitations}

An alternative method to ensure more accurate measuring and appropriate crediting for energy exports is to limit the types of DPV-plus-storage design configurations eligible for interconnection. These approaches typically involve limiting the ability of the storage system to import from or export to the grid using system controls. Common design configuration limitations include:

1. Storage can only charge from DPV, no storage exports allowed-a limited configuration that constrains the use of stored DPV energy for self-consumption only, and does not allow grid charging of the battery; the only energy eligible for credit under the DPV compensation mechanism is DPV energy that is produced and immediately exported to the grid.

\footnotetext{
${ }^{48}$ Violating NEM integrity principles here refers both to the misallocation of NEM credits to non-NEM-eligible generation and to the potential for arbitrage or relying on price differences in the retail rate of electricity to profit by buying energy during less expensive periods and selling it back to the system during more expensive periods. CPUC found that "[w]hile arbitrage is a theoretical concern under both methods ... [it] would be uneconomical based on current battery costs, current differentials in TOU period pricing, and round trip efficiency losses of 10-20\%" (CPUC 2016b, 20).
} 
2. Storage can charge from DPV and grid, no storage exports allowed - this configuration allows the customer to charge from both the DPV system and the grid, but the storage system is not able to export to the grid. Only DPV energy that is not immediately used or stored is exported to the grid and credited.

3. Storage can only charge from DPV, storage exports allowed-a less limited configuration where the storage device is allowed to export stored DPV-originated energy to the grid and receive credit under the DG compensation mechanism.

4. Storage can only charge from the grid, storage exports allowed - a limited configuration where the storage device is allowed to charge from and export to the grid, but is not allowed to interact with the DPV system.

In general, design configuration limitations ensure that any customer exports to the grid come exclusively from on-site generation, eliminating concerns of compensation mechanism integrity and energy arbitrage using gridsupplied energy. But only allowing the storage system to charge from the paired DPV system limits the ability of the energy storage system to become grid-interactive and provide valuable system services to the utility distribution system (e.g., congestion management) or the transmission network (e.g., frequency regulation). As opportunities expand for DPV-plus-storage systems to derive value from providing the broader power system with energy services (see Section 8.6), these limited design configurations may result in a lost opportunity in the future for both system owners and utilities - thus, limited design configurations should be considered with both a short-term and long-term lens.

\section{Jurisdiction Examples}

Hawaii-In Hawaii, NEM was closed to new participants in October 2015 and replaced with a transitional market structure featuring two interim systems and associated tariffs, which limited the amount and time at which energy could be exported to the grid from customers. Under the Customer Self-Supply option, customers who install DPV or DPV-plus-storage systems in configurations, which preclude exports to the grid, require interconnection review prior to installation, but are eligible for expedited review processes, including in areas that have already exceeded voltage limitations due to higher solar photovoltaic penetrations (HECO 2019a). This tariff incentivizes customers to install storage to maximize the consumption of their on-site DPV resource - the output of which may or may not naturally coincide with customer demand - and reduce their own consumption from the grid.

California-For DPV-plus-storage systems with batteries larger than $10 \mathrm{~kW}$ in California, regulators determined the estimation methodology used for smaller systems would not be sufficient to ensure NEM integrity and discourage arbitrage activities. These systems were instead required to use either a limited design configuration or additional metering (CPUC 2014). For the limited design configuration option, systems must install a nonexport relay on the storage device, which excludes the exporting (and thus, crediting) of grid-charged energy back to the grid, ensuring the actual amount of NEM-eligible generation can be accurately calculated (CPUC 2014). Regulators also instituted a battery sizing requirement for all batteries larger than $10 \mathrm{~kW}$ to ensure its primary functions are to augment the value of the paired NEM-generator and satisfy on-site demand, instead of performing arbitrage activities using TOU rates. The maximum output power of these paired storage systems may not exceed $150 \%$ of the paired NEM-generator's maximum output capacity, and the storage system's energy capacity is limited to a maximum of the energy equivalent of 12.5 hours of discharge at the maximum power capacity. ${ }^{49}$ The NEM-generator, in turn, was originally limited to $1 \mathrm{MW}$ in capacity under the original NEM tariff, but under California's new net metering scheme (NEM 2.0) there is no maximum size limit, although all systems over 1 MW are required to pay associated interconnection fees (CPUC 2016a).

Colorado-As with larger systems in California, Xcel Energy in Colorado only grants Renewable Energy Credits (described further in Section 8.3) to NEM-eligible renewable generation systems that are either configured so as to prevent the export from the battery to the grid, or that have adequate metering in place to appropriately assign Renewable Energy Credits (Xcel Energy 2018, 191-92).

Nevada-In 2018, the Nevada Public Utility Commission passed an interconnection rule that restricted energy storage devices paired with NEM-eligible DG systems such that they are either: (1) technically restricted from

\footnotetext{
${ }^{49}$ For example, if a storage system had a power rating of $4 \mathrm{~kW}$, then its energy capacity rating could not exceed $50 \mathrm{kWh}$.
} 
exporting energy to the distribution system altogether; or (2) technically restricted to being charged only from the customer's NEM-eligible DG system (NV Energy 2018a, 18).

New York-Although New York is transitioning toward its Value of Distributed Energy Resources tariff structure, at the time of this writing it still relies on a NEM framework, and concerns have been raised over distributed storage's potential to game NEM crediting schemes. To address these concerns, the New York State Department of Public Service approved a Hybrid Energy Storage System Tariff for DPV-plus-storage systems which would qualify for NEM credits (NYPSC 2018b). The tariff allows four different qualifying configurations, two of which involve limited configurations which demonstrate systems exclusively charge from eligible DERs and limit exports of grid-supplied power.

\subsubsection{Require Adequate Metering to Track All Quantities Relevant for Preserving Compensation Mechanism Integrity}

A final method for ensuring compensation mechanism integrity is requiring additional metering equipment. Installing additional metering to track the flow of energy within DPV-plus-storage systems is undoubtedly a robust method for preserving compensation mechanism integrity. It may also enable grid interactivity and participation in (likely not-yet-developed) grid services schemes (e.g., a virtual power plant scheme); however, a requirement for additional metering beyond what is needed for grid-tied DPV systems may incur significant costs, depending on the customer, particularly in the case of retrofitting existing systems with new meters. These costs may outweigh the benefits associated with more accurate measurements, particularly for smaller residential systems, which have a limited capacity to engage in energy arbitrage activities or to profit from selling gridsupplied energy back to the grid using DG compensation mechanisms.

\section{Jurisdiction Examples}

California-As an additional option for $>10 \mathrm{~kW}$ storage systems beyond design configuration limitations, regulators offered additional metering as an alternative means of ensuring NEM integrity. For DPV-plus-storage systems with batteries larger than $10 \mathrm{~kW}$, system owners had the choice to "[1)] install an interval meter for the NEM-eligible generation, meter the load and meter total energy flows at the point of common coupling; or [2)] install interval meter (sic) directly to the NEM-eligible generator(s)" (CPUC, 2014). Both of these methods ensure the actual amount of NEM-eligible production and exports can thus be accurately calculated for billing and goaltracking purposes.

New York-In addition to the limited design configurations listed above, New York's Hybrid Energy Storage System Tariff also allows for systems with sufficient metering equipment to qualify for NEM credits. For paired systems with appropriate telemetry and metering infrastructure on the storage system, credits are calculated by taking the difference of the net hourly injections measured at the customer meter and any discharges recorded on the storage system meter (NYPSC 2018b).

Massachusetts - Balancing the need for accurate solar energy tracking and the types of technologies and their costs capable of doing so is playing out in solar and storage programs today. In 2017, the Massachusetts Department of Energy Resources established the Solar Massachusetts Renewable Target Program to incentivize solar statewide. Eligible storage systems co-located with solar can receive an incentive adder (Department of Energy Resources 2017). To qualify for the program, solar-plus-storage customers are required to purchase a utility meter that measures their solar production. This requirement has prompted formalized concerns from installers that: (1) the cost of additional metering eats into incentive savings, reducing potential deployment; and (2) additional metering is unnecessary, because certain advanced inverters are capable of accurately tracking solar generation for Solar Massachusetts Renewable Target program compliance. Utilities contest the magnitude of additional metering costs and prefer revenue-grade metering technology. The Massachusetts Department of Energy Resources has yet to determine if advanced inverters are an acceptable alternative to additional metering for the SMART program (Spector 2019).

Behind-the-meter energy storage systems are dynamic and flexible energy resources, able to act as both a source of power and energy during some time periods while being a load with demand and energy needs at other time periods (or may be neither source nor load at other times). The technical design and configuration of such systems affect their ability to interact with the grid. Taking a purely technical perspective, storage systems can be designed 
to charge from the grid and/or an on-site distributed energy resource (e.g., DPV), and the charging source and/or duration may be limited by the distribution system operator. Similarly, they can also be designed to discharge to the grid, serve on-site customer electricity demand, or both through value stacking.

In reality, however, behind-the-meter energy storage system design configurations are often (though not exclusively ${ }^{50}$ ) influenced by a desire to reduce the hosting customer's electricity bill or provide reliability services during outages, which is influenced by how retail tariff structures and distributed energy resource compensation mechanisms are designed. When serving on-site power and energy needs, these systems might be technically optimized to reduce monthly power demand charges, take advantage of TOU tariffs, maximize DPV selfconsumption (when paired with a storage system) and avoid power and energy exports, and/or provide backup during outages or periods of grid instability. In any case, this technical design flexibility brings both challenges and opportunities for system owners, utilities, and the power system at large that utility regulators can understand to fairly and effectively regulate this technology.

\footnotetext{
${ }^{50}$ In some cases, the design and operation of third-party owned BESS may be optimized by developers to provide a specific type of bankable grid service (e.g., demand response in a power market).
} 


\section{Step IV: Design Interconnection Rules}

\section{Key Insight for Indian Stakeholders}

This section focuses on the role that regulators can play in the development of interconnection rules, which govern under what conditions a behind-the-meter system is allowed to interconnect to the power system. The section maps the potential set of administrative rules for how interconnection applications must be submitted and processed, technical rules governing equipment performance requirements for DPV-plus-storage systems, and administrative rules on how interconnecting systems are reviewed once an application is received. As much of the interconnection process may exceed the institutional capacity of some regulatory bodies, this section also focuses on the roles of the relevant parties in the development of these rules.

In India, much of the existing rules surrounding interconnection are focused primarily on DPV systems. Furthermore these existing rules may not adequately cover issues such as required communication infrastructure that may be necessary for more grid-interactive objectives. However, these rules can be straightforwardly amended to incorporate specific considerations for DPV-plus-Storage systems and help enable safe and orderly deployment of behind-the-meter storage devices.

From a practical standpoint, taking full advantage of the potential grid support benefits of behind-the-meter batteries in India will require a high standard of quality for system components, the design, installation, and maintenance of systems, and potentially communication infrastructure between battery inverters and system operators (see Section 6.2). Existing distributed solar quality issues in India point to potential challenges with achieving these standards with a new technology like batteries until sufficient local capacity is built (Jaiswal et al. 2017). Quality-related issues with battery systems for backup power applications will primarily only impact the customer; however, for battery systems providing passive grid support services (e.g., voltage ride-through), quality-related issues could conceivably cause larger power system complications if battery systems behave in ways DISCOMs and system operators are not prepared for-thus, procedures to verify that inverter settings are calibrated correctly may be worthwhile for SERCs to explore. In future scenarios where batteries are called upon directly by system operators (e.g., responding to a signal to inject power into the grid), additional quality control procedures may be required to ensure communication between the battery and system operators is secure and reliable.

One important regulatory undertaking to promote robust interconnection rules for DPV-plus-storage would fall to the Central Electricity Authority (CEA), which could consider updating the 2013 Technical Standards for Connectivity of the Distributed Generation Resources (CEA, 2013) regulations to include appropriate grid requirements for behind-the-meter storage and DPV-plus-storage systems. The CEA's previous document laid out a variety of widely utilized connectivity conditions, standards and codes of practice, and other procedural requirements for installing distributed generation resources which are frequently referenced by SERCs. These CEA regulations were recently amended in February 2019, extending their purview beyond distributed generation resources to also include "prosumers, when acting as a generator." The amended regulations make general accommodations for prosumers and grid-interactive charging stations applying for interconnection, and state that "the appropriate licensee shall carry out adequacy and stability study of the network before permitting connection" (CEA 2019b); however, these regulations could be further expanded and customized to make specific accommodations for behind-the-meter storage and DPV-plus-storage systems, including by providing a pathway for more streamlined interconnection for smaller-scale grid-interactive systems.

Concurrently, the Forum of Regulators could convene relevant stakeholders to develop model interconnection rules and regulations. SERCs can then direct DISCOMs to begin developing appropriate technical requirements for behind-the-meter storage based on these model regulations (see Sections 6.2 and 6.3). The Draft Model Regulation for Grid Interactive Distributed Renewable Energy Sources suggests that SERCs should assign distribution licensees the responsibility to assess the impact of various forms of storage systems on the distribution system (FOR 2019). Explicitly directing DISCOMs to engage with and assess storage systems can help these organizations better understand storage technologies and develop appropriate technical requirements that take into consideration the unique set of opportunities and challenges they present. 
Interconnection rules govern both the procedural elements and technical requirements associated with connecting a DPV-plus-storage system to the power system. They also communicate a variety of expectations to utilities, developers, and installing customers. Regulators can play an important role in guiding the overall development of DPV-plus-storage interconnection rules, which can be understood through the following categories:

- Rules governing interconnection application procedures and management offer guidance on the process for how developers and utilities must interact and specify the various steps that each stakeholder must take in the overarching process of formulating, evaluating, and ultimately approving or rejecting applications to interconnect to the power system. Specific rules about the interconnection process may include aspects such as mandated time frames for utilities or developers to take various steps (e.g., screen an application, respond to an inquiry), standardized data requirements that developers must provide to utilities in an application, requirements of how much information utilities must provide developers about the process, standardized terms of interconnection agreements that are ultimately executed between the customer and utility, schedules of interconnection fees, and dispute resolution processes, among others.

- Rules governing grid requirements offer guidance on which electrical equipment standards that system components must comply with, the technical configuration, metering, monitoring and control requirements, and the various technical requirements for how the system should be interacting with the power system during normal and abnormal operating conditions. Many model equipment standards (e.g., UL 1741) and interconnection standards (e.g., IEEE 1547) already exist in the United States for DERs, and regulators are often tasked with directing utilities to draw from these existing resources and/or engaging with the utilities and the public to adapt these resources to local conditions. When considering grid requirements, regulators typically attempt to balance a desire for: (1) the safety of utility workers and the general public; (2) the reliability and service quality of the distribution system; and (3) the affordability of equipment (and associated installation labor) needed to comply with requirements.

- Rules governing interconnection application technical review practices offer guidance on the technical criteria and associated procedures that utilities must utilize when evaluating an interconnection application, with a specific focus on how the proposed DPV-plus-storage system will impact operations on the distribution circuit where it will be constructed. This includes various screening criteria for when a particular application can be fast tracked for approval or may be flagged for supplemental or formal engineering review. In this realm, regulators are typically balancing a desire to avoid adverse distribution system impacts with a desire to ensure evaluation processes and criteria are not unnecessarily burdensome for consumers. Regulators may also provide guidance on cost allocation in the event that more expensive engineering studies or network upgrades are required to permit interconnection.

The design of interconnection rules involves the codification of regulatory decisions surrounding compensation mechanism design and metering and technical configuration requirements discussed in previous sections. While their exact role can vary greatly from region to region, regulators are increasingly playing a leading role in the adaptation, adoption, and public dissemination of existing interconnection standards and codes. Figure 12 illustrates the typical high-level process of navigating DER interconnection and permitting, beginning with application submission and ending with the utility granting the system permission to operate. The figure also highlights which authority or actor typically oversees each step of the process, as well as examples of which rules, requirements, standards, and codes the storage or DPV-plus-storage system may be subject to at that particular step. It is important to note that the rules included in the figure are intended as examples, and systems may be subject to different and/or additional rules depending on the jurisdiction. As well, the term authority having jurisdiction (AHJ) is used to describe any local authority that has oversight or control over a relevant permitting process - issues related to permitting and AHJs are discussed in Section 7. 


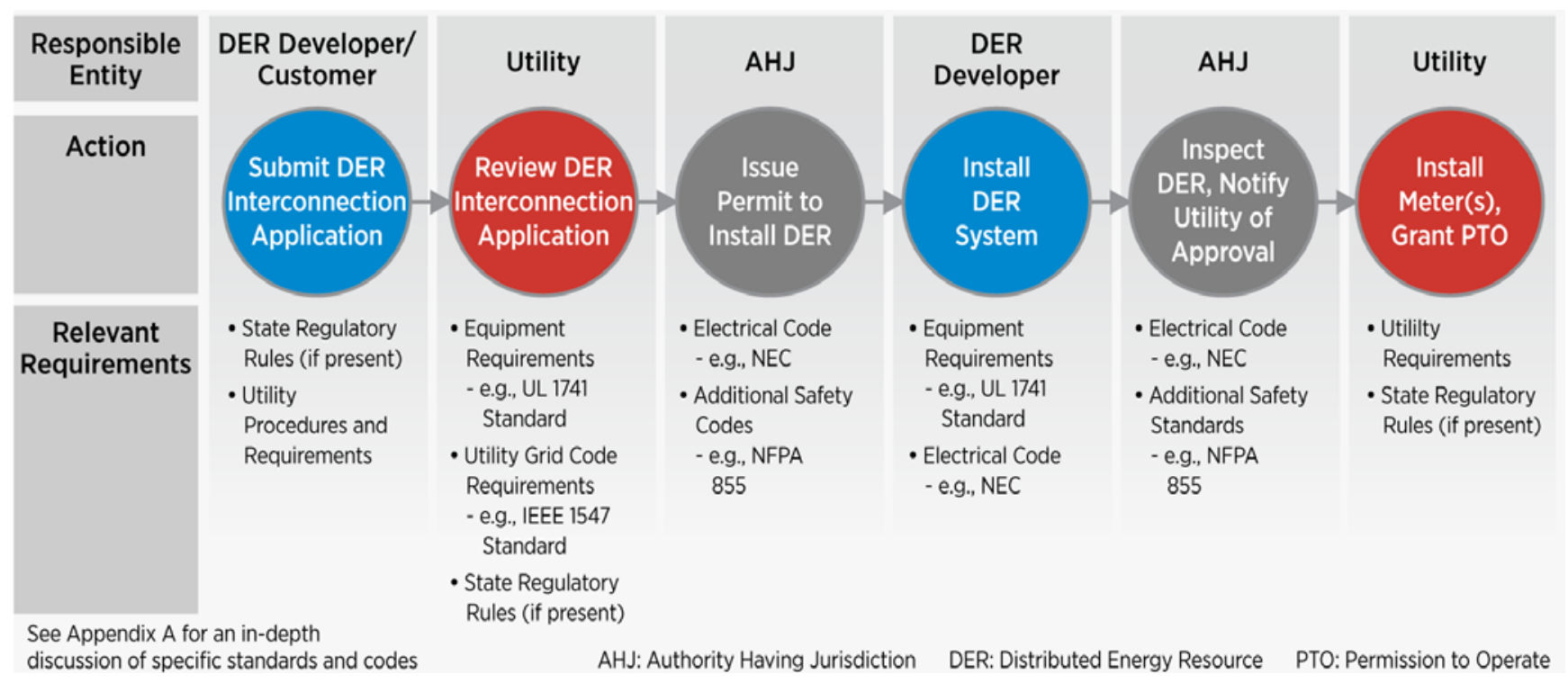

Figure 11. Typical DER interconnection and permitting process

Source: Original [IIlustration by Alfred Hicks, NREL]

Importantly, many regulators attempt to create standardized processes, applications, and technical requirements across their jurisdiction; doing so may help to ensure all applications are reviewed on a nondiscriminatory basis, and also reduces the need for developers to learn an entirely new set of interconnection procedures and requirements for each utility service territory. ${ }^{51} \mathrm{~A}$ key trend in creating such standardized processes is to customize interconnection rules based on system capacity (see Section 2.2), with smaller systems being subject to less rigorous interconnection requirements.

In the United States, regulators have pursued several distinct approaches to address interconnection processes in response to increasing DPV-plus-storage deployment. These include:

- Actions which clarify that existing interconnection rules for small-scale distributed energy resources apply to storage (e.g., California, Colorado, Iowa, North Carolina, and New Mexico);

- Actions which develop separate interconnection rules for DPV-plus-storage systems to address some of the unique operational features of storage (e.g., California and Nevada), including:

○ Actions which distinguish between non-exporting and exporting DER systems and apply exceptions or modifications (e.g., expedited review, limited review, or no review) for nonexporting systems that pose less risk to the grid (e.g., California, Colorado, New York, Hawaii, Arizona [proposed]); and

- Actions which customize the rigorousness of interconnection rules, with specific technical requirements, including applicable standards and codes, based on the size of the applying system (e.g., New York, California).

\subsection{Rules Governing the Interconnection Application Procedures and Management}

Interconnection processes for DERs have been discussed at length in the literature (see Horowitz et al. (2019)) and have also been promulgated by utilities and regulators (see NYPSC (2017)). In practice, DPV-plus-storage interconnection processes may be quite similar to (or even the exact same as) processes for grid-tied DPV systems. While their exact role can vary greatly from region to region, regulators may be interested in addressing

\footnotetext{
${ }^{51}$ In some cases, utilities can have additional utility-specific requirements not specified (but authorized) by the regulator.
} 
some or all of the following set of issues related to interconnection application procedures and management (this section draws heavily on Horowitz et al. (2019)):

\section{Interconnection Pre-Application Rules}

- Public Communication of Interconnection Process: Regulators may wish to ensure that information about DER interconnection processes are broadly available to the public. This may involve requiring utilities to: (a) post relevant information on their website, such as frequently-asked-question pages or other customer-facing documentation that describe the various steps in the application process, data requirements, timelines, and fees; (b) provide reference materials such as example application documents, sample line diagrams, technical requirement documentation, or instructional videos; and (c) host trainings or webinars for developers and customers. In many cases, utilities may take these (or similar) steps without regulatory intervention, but regulators may nevertheless wish to set a minimum standard for public outreach and communication.

- Availability of Data on Interconnection Feasibility ${ }^{52}$ : Regulators may wish to expand the availability of information on the technical feasibility of interconnection at various points on the utility distribution system. This can occur through the issuance of pre-application reports, where developers request the utility to provide readily available information on the potential limitations of a specific distribution circuit where they are considering developing a project. In this case, regulators may need to help determine a reasonable cost that utilities can charge developers for this service, as well as mandate a time frame for providing the report. Regulators may also wish to ensure utilities publish and periodically update DER hosting capacity maps, which visually communicate technical information on the feasibility of DER interconnection to the public, such as is currently available from California investor-owned utilities. ${ }^{53}$ Hosting capacity maps can help gridtied DPV and DPV-plus-storage developers understand the maximum capacity of DER systems that can be installed in a specific location, saving developers time and energy by avoiding areas with lower probabilities of successful interconnection. For more information on hosting capacity maps and analysis, see the text box in Section 6.3.2.

\section{Interconnection Application Rules}

- Utility-Developer Communication: Utilities and developers/customers may need to interact throughout the interconnection process to address various requests and questions. Regulators may wish to ask utilities to provide a single point of contact for each applicant to answer interconnection-related questions or guarantee the right of applicants to request scoping meetings with utilities to discuss interconnection requests and review applications. Such requirements can help to reduce developer uncertainty, streamline communication, and limit confusion on application statuses and next steps.

- Interconnection Application Form Design: Regulators may wish to directly design, provide input to, and/or formally review and approve interconnection application forms, which provides a variety of relevant data to utilities about prospective projects. Typically, regulators aim to ensure that applications are sufficiently clear and understandable for customers and provide a reasonable amount of information to utilities to evaluate requests. In some cases, larger systems may merit separate application forms with additional information requirements.

- Application Submission Procedures: Regulators are often concerned with ensuring a fair and transparent process for submitting and evaluating applications. In the realm of fairness, utilities may be required by regulators to process applications on a first come, first served basis in the event that multiple applications are submitted for projects on the same feeder. Regulators may also require utilities to offer an online application submission process and/or an online application status tracking tool. They may also wish to specify utility protocols in the event of an incomplete application submission by a developer.

\footnotetext{
${ }^{52}$ It is important to note here that feasibility refers broadly to the ability of a prospective grid-tied DPV or storage-plus-DPV system to be interconnected to the power system without compromising reliability or resilience. This is independent of the more industry-specific definition of a feasibility study, which larger utilities may require for larger, front-of-the-meter installations.

${ }^{53}$ For two examples of these maps, register here for PG\&E: https://www.pge.com/en_US/for-our-businesspartners/distribution-resource-planning/distribution-resource-planning-data-portal.page, or here for SDG\&E: https:/www.sdge.com/more-information/customer-generation/enhanced-integration-capacity-analysis-ica.
} 
- Application and Study Fees: Regulators typically specify or review utility proposals for customer fees that may be charged when interconnection applications are submitted and/or additional engineering studies by the utility are required.

\section{Interconnection Process Rules}

- Overarching Structure of Interconnection Process: Regulators are typically involved in developing the overall progression of steps in the interconnection process, including aspects such as (but not limited to) initial application submission, preliminary application reviews, utility technical screening, supplemental engineering studies (if merited), distribution system mitigation upgrades (if merited), project construction, system testing and certification, and final system verification. In practice, a given interconnection process can go in a number of different directions, depending on how an application progresses through the various steps, and what types of additional utility-developer interactions and/or additional engineering studies are required. Regulators may wish to play a role in developing a standardized utility process that helps streamline the overall process and reduce developer uncertainty.

- Mandated Time Frames for Interconnection Process: Regulators are often focused on mandating the maximum allowable time frame for utilities and developers to take various steps in the interconnection process, such as a maximum response time for a utility to evaluate an application or conduct an engineering study, or for a developer to respond to a request for more information from the utility. Creating guidelines for response times from utilities and DER developers helps to reduce uncertainty for all parties, ensuring that applications waiting in a queue for a particular distribution circuit are not unnecessarily delayed.

- Dispute Resolution Processes: Regulators may wish to specify a predetermined procedure, which is followed in the event that a dispute occurs between the utility and developer related to the implementation of the interconnection process. This process is typically specified within an interconnection agreement. Regulators may provide general guidance on the location, time frame and method for dispute resolution (e.g., enrollment of a mutually acceptable mediator in a mutually convenient location within 30 days), and/or may interject as the mediating authority themselves if a resolution cannot be met through the initial process.

\section{Screening Rules}

- Technical Screening Procedures: This issue will be discussed in additional detail in the Section 6.3.

\section{Final Interconnection Rules}

- Terms of Interconnection Contract: Regulators may directly design, provide input to, and/or formally review and approve utility- or jurisdiction-specific interconnection contracts. These contracts outline the terms of the legal agreement between the utility and the customer, allowing the customer to sell electricity back to the utility and detailing a variety of rights and restrictions that utility and customer are subject to. In some jurisdictions, regulators directly promulgate standardized interconnection contracts that balance utility and customer interests.

- Cost Allocation for Grid Upgrades: In the event that a distribution system upgrade is required to accommodate a prospective DER, regulators must specify who is responsible to pay for the cost of the upgrade. Deciding how upgrade costs can be allocated equitably can be a challenging task. ${ }^{54}$ This is typically addressed through the promulgation of a broadly applicable set of regulatory rules.

\footnotetext{
${ }^{54}$ See Section 6 of Horowitz et al. 2019 for more information on this topic.
} 
The relative role of regulator versus utilities in creating these rules is summarized in Table 3.

Table 3. Roles and Responsibilities for Interconnection Application Procedures and Management Rules

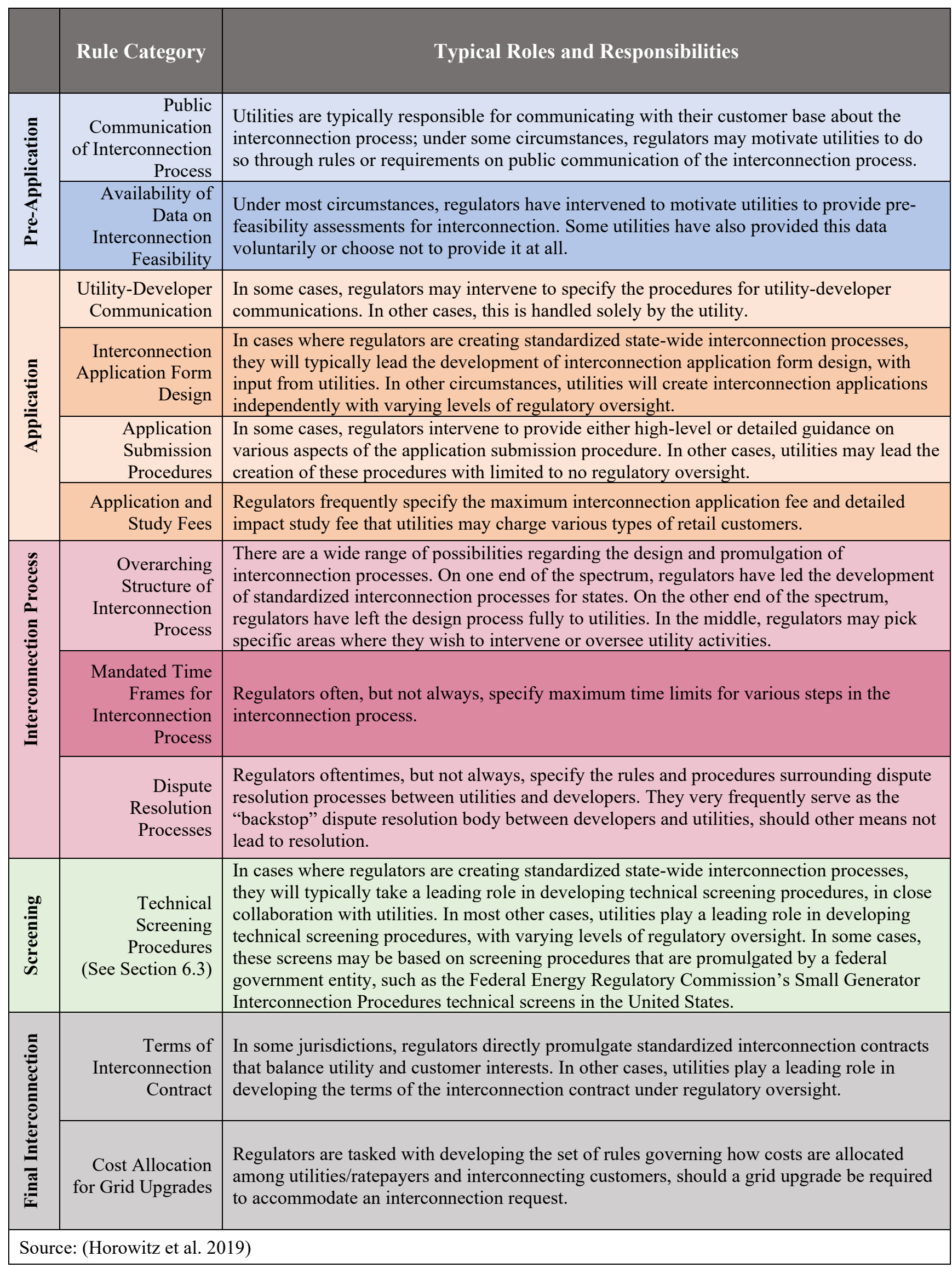




\subsection{Rules Governing Grid Requirements}

In addition to rules governing interconnection application procedures and application evaluation protocols, the promulgation of grid requirements is an important element of regulating interconnection processes. Grid requirements typically comprise equipment requirements, which govern technical capabilities and quality of DER equipment, and interconnection requirements, which govern the interactions and interoperability of equipment with the broader power system.

The establishment of grid requirements within a given regulatory jurisdiction helps to establish the minimum technical parameters that DPV-plus-storage systems must exhibit to safely interconnect to an electrical power system. Their implementation helps to ensure standardized, predictable behavior from all interconnected distributed assets, which safeguards against DER interfering with the safe operation of the power system, enables DER to help restore the power system to normal operating conditions during frequency and voltage excursions, ensures the safety of utility line workers, and allows DER to contribute to the broader reliability of the power system given adequate facilitating infrastructure (see Sections 8.5 and 8.6 for examples of DER providing system energy and ancillary services). Regulators have an important role in the development and adoption of grid requirements, including the adaptation of established international equipment and interconnection standards to local power system conditions, and the regular update of interconnection requirements to keep pace with changing system conditions.

In general, equipment requirements and interconnection requirements rely on established standards developed by national and international standard-setting organizations (e.g., Institute of Electrical and Electronics Engineers [IEEE]) and safety certification bodies (e.g., Underwriters Laboratories). Interconnection and equipment standards themselves are voluntary guidelines that establish basic procedures and minimum technical performance metrics for equipment and systems operating in parallel to (i.e., grid-tied) or in isolation from (i.e., stand-alone or off-grid) the power system. They are often adopted directly or modified as needed by regulators and utilities to form mandatory interconnection requirements and equipment requirements that comprise a broad set of grid requirements.

\subsubsection{Interconnection Requirements and Underlying Relevant Standards}

While requirements for interconnecting larger, grid-tied systems may be processed on a case-by-case basis, for smaller distributed systems, these requirements are often codified in technical requirement documentation known as interconnection requirements. Interconnection requirements are key mechanisms that utilities use to ensure safe and reliable interconnection processes when connecting new DERs (Nagarajan 2018). The requirements are primarily focused on the interface between DERs and the power system. DER interconnection requirements govern behavior related to:

- the operation of the DERs during normal grid operation

- the response of the DERs during abnormal grid conditions

- physical safety, maintenance practices, and communication cybersecurity protocols

- the ability of DER to communicate through accepted protocols

- the interoperability of DER systems to streamline their operation and interconnection (Nagarajan 2018).

The requirements do not typically offer guidance on the operation or planning of the power system in the context of DERs.

DER interconnection requirements will typically establish a minimum standard of behavior for components related to specific actions to help maintain or re-establish system stability. For instance, a DER interconnection requirement may include a requirement dictating the minimum ability of a DPV-plus-storage system to absorb or inject reactive power into the electric distribution system or the ability to stay online during system disturbances. Often, DER interconnection requirements designate a range of latent capabilities that interconnecting systems must be able to provide, such as IEEE 1547's performance categories - ultimately, utilities (sometimes under the 
oversight of regulators) will choose to activate the capabilities and responses most appropriate for their power system context within a mandatory interconnection requirement.

Most DER interconnection requirements are to some extent underpinned by the voluntary industry standard IEEE 1547, the most recent of which was updated in 2018. IEEE 1547-2018, or Standard for Interconnection and Interoperability of Distributed Energy Resources with Associated Electric Power Systems Interfaces, is a voluntary industry standard for DER interconnection that establishes criteria and requirements for interconnection of DER with electric power systems and associated interfaces (Horowitz et al. 2019; Nagarajan 2018). IEEE 1547 is generally technology neutral and refers to all DER connecting to the power system, including both grid-tied DPV and DPV-plus-storage systems. To meet IEEE 1547-2018, DER systems and their individual components must be capable of meeting all performance categories and communication protocols. In reality, some utilities and regulators that use IEEE 1547 as a basis for their interconnection requirement may select which of the latent capabilities to activate according to local power system characteristics and needs. Others may choose to adopt IEEE 1547 as their interconnection requirement wholesale—without any major modifications to the various design parameters.

\subsubsection{Equipment Requirements and Underlying Relevant Standards}

One of the most important aspects of guaranteeing the safety of utility line workers and the public more broadly is ensuring that all interconnected electrical equipment operates as required and is built to comply with relevant interconnection requirements and electrical codes. To ensure safety, electrical codes governing the construction of systems behind the utility meter (see Section 7), as well as interconnection requirements which govern interactions of DER with the power system, require electrical equipment be certified by a recognized testing and certification laboratory, such as Underwriters Laboratory in the United States, which promulgates electrical testing requirements and certifies equipment from various manufacturers. Equipment standards developed by such institutions typically consist of a series of testing procedures certifying that a specific piece of equipment undergoing certification is capable of behavior required by common interconnection standards. While interconnection standards, such as IEEE 1547, may dictate the behavior required from inverter-based systems and DERs interconnecting to the power system, the testing to certify that inverters are actually capable of such behavior is contained in a separate equipment standard known as UL $1741 .^{55}$

Equipment precertification helps ensure that individual pieces of equipment and/or common combinations of equipment (e.g., solar panels, batteries, inverters, and/or other interface devices) are acceptable for continuous operation and interaction with the utility grid and can be reviewed and approved in a streamlined manner. While using certified equipment can help to avoid costly or time-consuming equipment reviews and tests, this does not automatically qualify an interconnection application for approval-relevant screening criteria must still be implemented, and interconnection requirements must be complied with as well.

\subsubsection{The Role of Regulators}

IEEE 1547 and UL 1741 have been adopted in some form by the majority of U.S. states as binding interconnection requirements and equipment requirements. Yet, regulators in each jurisdiction have been faced with decisions on how exactly to adopt and incorporate them into their grid requirements, as these standards allow for some customization of desired DER behavior. Some U.S. regulators, often with a statutory mandate motivating them, have played a leading role in developing and implementing state-wide grid requirements. ${ }^{56}$ Other regulatory bodies lacking statutory mandates, adequate staffing resources, and/or institutional capacity are frequently tasked with reviewing, modifying (if warranted), and approving utility proposals for grid requirements in public regulatory proceedings. In these circumstances, regulators may also provide broad guidance to utilities, such as to modify certain characteristics of particular standards.

\footnotetext{
${ }^{55}$ Interconnection standards and equipment standards often develop in tandem or in response to one another.

${ }^{56}$ In the United States, in jurisdictions that have seen significant DPV and storage-plus-DPV deployment (e.g., Hawaii Rule 14H and California Rule 21 - see Appendix C), regulators have chosen to play a more significant role in the development of standardized state-level interconnection requirements, whereas elsewhere regulators have adopted standards such as IEEE 1547-2003 and UL 1741 with little to no changes.
} 
Regardless of whether existing standards are adopted or modified, regulators also have an important role in ensuring that interconnection requirements are publicly available. Clear, transparent, and readily accessible grid requirements can ensure a smooth deployment of DPV-plus-storage systems by communicating to customers and installers acceptable equipment and systems (e.g., UL-certified). Furthermore, regulators often aim to ensure a consistency of interconnection requirements across various utilities operating under their jurisdiction. This consistency can reduce administrative burden for installers by eliminating the need to learn multiple utility interconnection processes while doing business in a single regulatory jurisdiction (e.g., a state).

Importantly, regulations governing the grid requirements are not static, and regulators have an important role in determining when state and/or utility-specific requirements need to be updated in response to an evolving power system and market context. New technologies (e.g., behind-the-meter battery energy storage systems), improved capabilities (e.g., the ability for inverters to absorb and provide reactive power, even absent solar generation), and increasing levels of system deployments (which can exacerbate technical issues on the distribution system) are all reasons for updating existing equipment requirement and interconnection requirements. In the United States, updates to grid requirements have been driven primarily by:

- increasing penetrations of new inverter-based technologies and associated increases in bidirectional flow of power at the distribution level

- the availability of improved communication and controls for DER systems

- the need for increased power system flexibility due to increasing levels of variable renewable energy generation (Nagarajan 2018).

As the number of DERs on power systems has grown, these systems are increasingly being required to play a larger role in maintaining power system reliability. For instance, the 2003 version of IEEE 1547 required distributed assets to trip offline during abnormal grid events and barred these assets from providing any sort of voltage regulation because there was little experience with significant levels of DERs on the grid system at this time. In 2014, IEEE 1547a was published, requiring DERs be capable of actively regulating voltage, providing improved frequency ride-through, and riding through a wide range of high and low voltage levels. The standard was again updated in 2018 with additional details and requirements, such as allowing, but not requiring, DER systems to provide inertial response (Horowitz et al. 2019).

\subsection{Rules Governing Interconnection Application Technical Review Practices}

The technical review practices that govern interconnection are reviewed below, including the DER technical screening process, distinctions for screening DPV-plus-storage, and U.S. examples.

\subsubsection{Review of Distributed Energy Resource Technical Screening Processes}

Interconnection applications are subject to a technical review process by utilities prior to interconnection to ensure that the proposed DER system will not interfere with the safe and reliable operation of the distribution system. This review involves the implementation of various technical screening criteria (often referred to as screens) through a structured process. Regulators will typically either directly promulgate these screening requirements, or review and approve utility proposals to do so, or some combination thereof. ${ }^{57}$ These screens may either be developed by regulators and utilities themselves or may be adopted and adapted from model screens developed by other regulatory agencies, such as the U.S. Federal Energy Regulatory Commission (FERC) Small Generator Interconnection Procedures (SGIP).

Technical screening criteria, which are a series of technical questions about the proposed interconnected system, are intended to account for both the key operating parameters of the proposed interconnecting system as well as the actual conditions of the circuit to which the system intends to interconnect. Ultimately, these screens are

\footnotetext{
${ }^{57}$ In more advanced jurisdictions, regulators have taken a more hands-on approach to the development of the interconnection review process, such as in California, where regulators intend to require utilities to use hosting capacity analyses (see Hosting Capacity Analysis text box) to further fast track interconnection applications.
} 
designed to ensure that the level of review to which interconnecting systems are subjected is commensurate with their potential impact on the local distribution system. Technical screening criteria are typically framed in a technology-neutral format and often cover all generating facilities connecting to the distribution system, rather than grid-tied DPV systems or DPV-plus-storage systems independently. Thus, the same technical review process used for grid-tied DPV systems should adequately cover DPV-plus-storage systems, with few modifications (discussed in the subsequent subsection).

Once an application for interconnection is received by the utility, there may be a series of initial application review questions to quickly determine if the system requires a more in-depth review or might immediately require a more detailed study. One important concern for utilities is whether or not the system uses pre-certified equipment, such as inverters certified under UL 1741 (see Section 6.2.2 for a discussion on equipment requirements and standards), which guarantees a minimum threshold of safety and ensures the system will behave in a reliable and predictable manner. Using certified equipment is typically required for systems seeking expedited, or fast tracked, approval. Certified equipment, however, does not guarantee fast track approval.

In practice, systems will still be subject to initial technical review screens, usually in the form of simple, binary yes-no questions and statements, which cover fundamental operational behavior of the system (e.g., "Aggregated DG does not cause protective device to exceed $87.5 \%$ of short circuit interrupting capability"). Importantly, DPVplus-storage systems which are designed to not export to the distribution system may be subject to a lower number of technical review screens than exporting systems. For instance, Hawaii's Rule $14 \mathrm{H}$ states that installing customers who participate in the Customer Self-Supply program — which does not allow grid exports - are exempt from 5 of the 10 initial technical review screens that exporting customers are subject to (HECO 2015a). ${ }^{58}$

In many technical review processes, proposed systems that do not satisfy these qualifications may be subjected to a series of more in-depth supplemental review screens. If the proposed system passes these supplemental screens, then it may still qualify for a fast-tracked approval.

Systems that fail both initial technical review screens and supplemental technical review screens will be subject to a detailed impact study to determine the extent of expected technical impacts and what mitigation measures, if any, can and should be taken before the system can be allowed to interconnect. These studies are typically paid for by the DER applicant and are only conducted if the DER applicant agrees to pay for the study. Impact studies, which can be performed by the utility themselves or be required from third-party groups, can be quite costly, especially relative to the cost smaller scale DER systems. Regulators are therefore often tasked with determining a fair and appropriate fee that utilities can charge or require of customers for their completion, as well as a reasonable time frame for completing the study.

\footnotetext{
${ }^{58}$ Other key distinctions for screening storage-plus-DPV systems will be discussed in Section 6.3.2.
} 


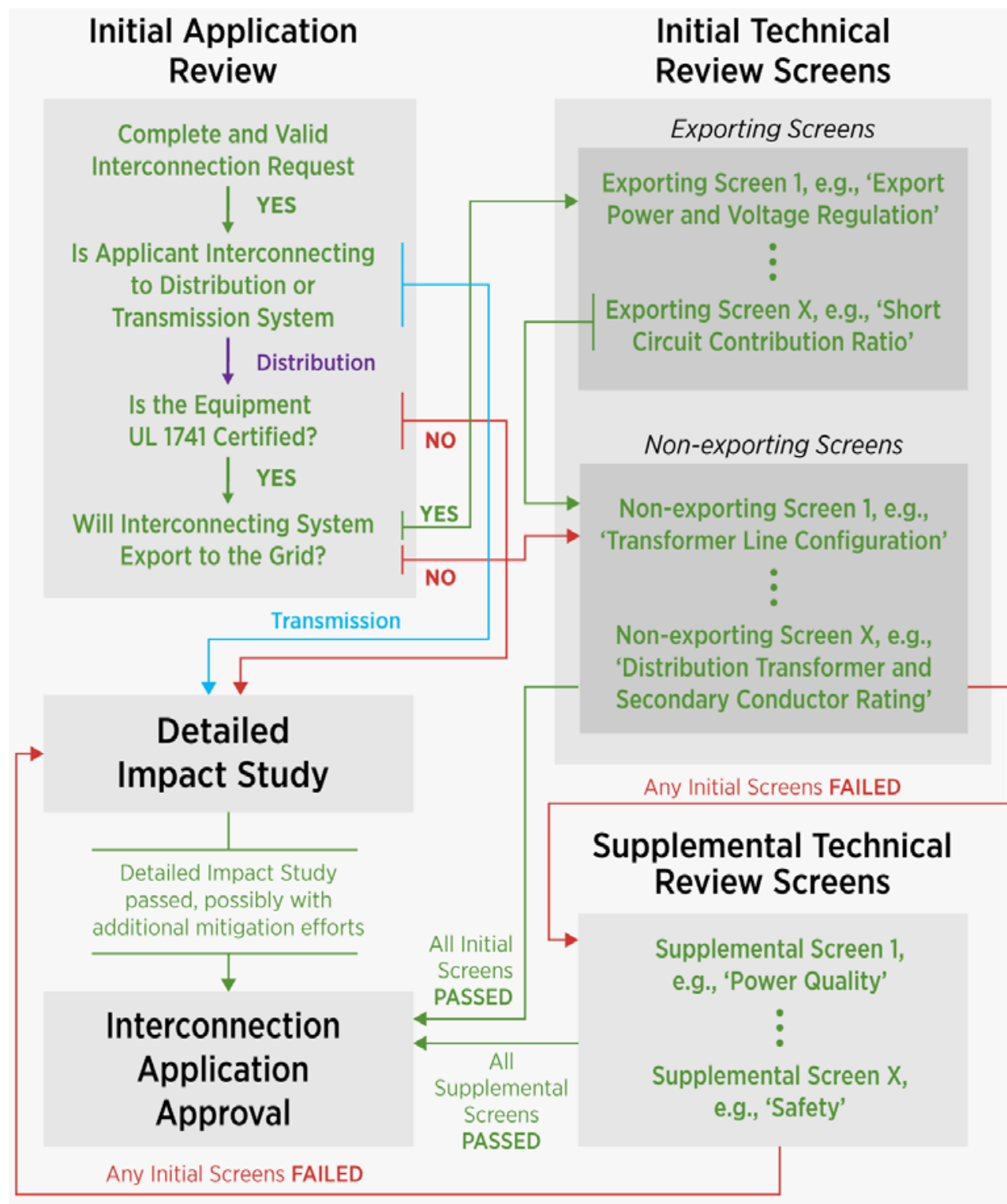

Figure 12. Overview of technical screening process for an interconnection application Source: Original [IIlustration by Alfred Hicks, NREL]

Managing a significant number of interconnection applications can become administratively burdensome for utilities, and subjecting each application to a detailed review may exhaust utility resources or cause application processing times to exceed regulated time limits. Categorizing interconnection requests through a series of increasingly more involved technical screens, as shown in Figure 13, helps reduce the administrative burden for utilities and average application times for customers without sacrificing the reliability of the power system. Furthermore, streamlining applications this way helps utilities focus their resources on reviewing proposed interconnections which are most likely to cause potential issues. Figure 13 depicts one example of the different levels of technical screens and how they interrelate and can ultimately lead to a detailed engineering study.

\subsubsection{Distinctions for Screening DPV-Plus-Storage Systems}

As mentioned previously, the same general technical review criteria and process used to evaluate grid-tied DPV systems can be used to evaluate DPV-plus-storage systems; however, there are a few key differences. Depending on the technical configuration, intended use case, and total export capacity of the DPV-plus-storage system, various changes to the technical screening process may be appropriate. 
To begin, screening criteria are often implemented using the nameplate capacity of the DER; however, it may not necessarily be appropriate to use this metric for a paired storage and DPV system. For example, if a DPV-plusstorage system's control settings limited its ability to charge from or export to the grid (e.g., due to a technical configuration requirement), evaluating the combined nameplate capacity of the battery and DPV systems may be inappropriately rigorous, and the DPV system capacity could instead be used. In the event that the storage system undergoing review is allowed to export to the distribution system, it is increasingly common in the United States to use a capacity metric that reflects the maximum possible export of the combined DPV-plus-storage system rather than nameplate capacity. This metric is often based on the inverter nameplate size, or if multiple inverters are involved, a combined nameplate size, in $\mathrm{kW}$ or kVA. Utilities in states such as Minnesota, Nevada, North Carolina, and South Carolina are required to evaluate the interconnection requests of DPV-plus-storage systems based on their net system capacity (i.e., the maximum export allowed by the storage control system), rather than the nameplate capacity (i.e., the aggregate capacity of the battery and DPV system) (see Nevada's Rule 15) (Horowitz et al. 2019). Along similar lines, Colorado's Xcel Energy and NV Energy's interconnection guidance documents also discuss how the net capacity of storage systems can be reduced using programming and controls (Xcel Energy 2017; NV Energy 2018a).

At a high level, understanding the intended use and technical configuration of a DPV-plus-storage system can help utilities and regulators determine which capacity metric should be evaluated to avoid unnecessarily rigorous review processes. Carefully considering which capacity metric to evaluate for an interconnection application is particularly important for systems, which are designed not to export to the grid, as these systems are least likely to interfere with normal operations and could reasonably benefit from being subjected less intense scrutiny (Peterson 2018; Horowitz et al. 2019). For instance, in Hawaii, customers who install DPV systems in configurations that preclude exports to the grid are eligible for expedited review processes (HECO 2019a).

\section{Jurisdiction Examples}

California-Rule 21 mandates the process and requirements for generation facilities to interconnect to the distribution system. Numerous revisions have been made over the years, including two recent revisions that clarify an expedited interconnection process for non-exporting storage and further explain the review process for evaluating load aspects of storage (an important factor in determining how costs are allocated if storage load triggers grid upgrades) (PG\&E 2018). Currently, Rule 21 is under revision to include, among other updates, the incorporation of a hosting capacity analysis and power flow analysis known as the Integration Capacity Analysis tool to further streamline interconnection application processing (CPUC 2017b).

Nevada-Rule 15 clarifies that net, not gross, system generating capacity will be used to determine storage system sizes and the requisite rigorousness of interconnection review. Moreover, non-exporting storage are required to undergo supplemental testing procedures to ensure their control systems function properly and comply with inadvertent export restrictions (NV Energy 2018a ).

Hawaii-Interconnection requirements address both non-exporting and exporting behind-the-meter DER systems in Hawaii, while also making special accommodations for retrofit storage systems (i.e., those added onto an existing DPV system) versus new-build systems. All exporting systems (e.g., those under Hawaii's Smart Export Program) follow the standard interconnection process (which includes provisions for evaluating storage capacity and exported power) (HECO 2015a, 14; 2018, 25). New-build, non-exporting systems fall under Hawaii's Customer Self Supply program and are subject to a reduced number of technical screening criteria relative to exporting systems. Non-exporting storage retrofitted to an existing, exporting NEM-approved DPV must register with the utility but generally bypass technical reviews (Peterson 2018); however, regulations state that "the total export capability must be limited to the size of the original NEM system," and advanced inverter requirements must be met (Hawaii PUC 2018, 33). If a customer chooses to add additional DPV capacity and storage to an existing NEM account, they would fall under Hawaii's NEM Plus program - provided that the original export capacity for which they have already been approved under the NEM program is not exceeded, they are subject to the less rigorous Customer Self Supply screening procedure. 


\section{Hosting Capacity Analysis}

DER hosting capacity is the total DER capacity that can be accommodated on a given distribution feeder, without any feeder upgrades or modifications, before adverse impacts to critical operational parameters (such as voltage, frequency, power quality) begin to manifest (Ding et al. 2016). Hosting capacity is an important parameter for utilities that clearly communicates whether a feeder is in danger of experiencing abnormal operation due to the presence of DERs. This metric is often displayed using distribution feeder maps that indicate the DER capacity that can be accommodated at specific locations throughout the distribution system. This metric and associated maps can help utilities quickly categorize interconnection applications based on the local conditions of the feeder to which the proposed system intends to interconnect. The hosting capacity of a feeder is not static and can change in response to many factors, such as how the various interconnected DERs are being operated, the presence of mitigating measures and equipment, or the consumption patterns of customers present on the feeder. For instance, the increased supporting role of DER that is enabled when the IEEE 1547-2018 standard is adopted can increase the DER hosting capacity of feeders by allowing smart inverters to mitigate potential voltage issues caused by PV arrays (Nagarajan 2018).

DER hosting capacity analyses use power flow studies to determine the maximum level of installed capacity of a particular technology that a feeder can support without interfering with normal grid voltage, protection coordination, and other essential quantities. These analyses can function as important tools for utilities to help accurately and quickly determine whether a proposed system (such as storage-plus-DPV) will negatively impact grid operations based on system and feeder specific characteristics (system capacity, number of customers on the feeder, intended use case of the system, and so on).

In some jurisdictions, regulators have begun incorporating these metrics and studies into the interconnection process to streamline application processing times while increasing the technical rigor of screening criteria. In California, regulators are currently revising their interconnection process to require utilities to periodically conduct hosting capacity analyses as a part of their standard technical screening process (CPUC 2017b).

Utilities will be required to supply periodically updated hosting capacity maps to developers, which can help developers target their installations in areas where interconnection is least likely to require expensive mitigation efforts. This can lead to less administrative burden for utilities tasked with processing these applications, as well as faster and less expensive interconnections for customers and developers. (To explore one of these maps for SCE, see this guidebook: https://www.sce.com/sites/default/files/inline-

files/DERiM_User_Guide_Final_AA_0.pdf, or visit this website: https://1tmdrpep.sce.com/drpep/.)

In Hawaii, the Hawaiian Electric Companies are required to provide circuit-level hosting capacity analysis for all islands in the Companies' service territories, and to make that information publicly available. Locational Value Maps, found on the Companies' website, for Oahu, Maui County, and Hawaii Island indicate the approximate amount of DPV currently on the utilities' primary distribution network, as well as the available remaining total capacity output available for customers to connect DPV to the grid based on the hosting capacity of the primary circuit and service address location. Maps can be accessed at https://www.hawaiianelectric.com/clean-energy-hawaii/integration-tools-and-resources/locational-value-maps. 


\section{Step V: Consider Local Permitting Issues}

\section{Key Insight for Indian Stakeholders}

This section focuses on the relevance of building wiring and fire safety codes in processes to interconnect DPVplus-storage systems. In India, state governments typically allocate responsibilities for enforcing wiring and fire safety codes for rooftop solar installations to some combination of the state-level Chief Electrical Inspectorate to Government (CEIG) and the interconnecting DISCOM. CEIG inspection is often mandated for larger distributed generation resources (e.g., $>1 \mathrm{MW}$ in Karnataka, $>200 \mathrm{~kW}$ in Maharashtra), whereas smaller systems are inspected by the DISCOM. In some cases, inspection may be waived altogether.

At a high level, the introduction of behind-the-meter DPV-plus-storage systems will likely require the development of additional wiring and fire safety codes (and associated inspection procedures and training for installers and emergency responders) that would be enforced by DISCOMs and state CEIGs in India. In practice, this will require efforts by relevant code-setting bodies to consider issues such as spacing, security (e.g., fencing for outdoor systems), placement of electrical disconnects and emergency stops, construction building or encasement material (e.g., noncombustible), fire suppression systems, and ventilation and exhaust systems for behind-the-meter storage systems. Of particular relevance in India may be the imposition of operational constraints on batteries when operating under higher ambient temperatures in order to avoid fire risks.

Both central and state governments in India can play a central role in developing and facilitating familiarity with new codes and inspection procedures for behind-the-meter storage - for instance, governments can promulgate best practice manuals for enforcing storage wiring and fire safety codes, and/or hold workshops to boost familiarity with inspectors.

While not directly responsible for them, regulators may need to account for the presence of local permitting processes and compliance with relevant electrical codes within interconnection processes for DPV-plus-storage deployment.

In the United States, local jurisdictions issue construction and building permits for DG systems as part of their requirement to ensure safety, public health, and general welfare as it relates to buildings and construction-in the electric utility sector in the United States these local jurisdictions are sometimes referred to as the AHJ. AHJs issue permits that recognize compliance with relevant electrical and fire codes adopted by the jurisdiction and signal to the utility that a DER system can be permitted to operate, provided it has successfully complied with the relevant grid requirements (for an overview of the entire interconnection and permitting process, see Figure 12 in Section 6). In the United States, the main electrical code adopted by jurisdictions is the NEC, which covers all elements relevant to the safe and secure installation of electrical equipment and electrical wiring. Where interconnection and equipment standards specify the desired operation of the DER, the NEC provides guidance on how to safely construct and wire a DER system.

With the increasing deployment of DPV systems, many local governments have increasingly adopted streamlined permitting procedures, and have gained familiarity with issuing permits for DPV systems. Few, however, have experience permitting behind-the-meter battery storage systems, leading to inconsistent permitting processes and fees across jurisdictions. Understanding if and how the permitting processes of stand-alone storage and/or DPVplus-storage systems differ from grid-tied DPV system permitting processes may be increasingly important as battery prices continue to decline and businesses and residents begin to deploy these systems at-scale.

To address this information gap, California passed Assembly Bill 546 in September 2017, directing California cities and counties to make storage permitting procedures and applications publicly available online and accept applications electronically. Additionally, the bill instructed the Governor's Office of Planning and Research (OPR) to educate communities and local governments on storage permitting and inspection best practices (California State Legislature 2017). Energy storage workshops held in 2018 informed the development of an 
energy storage guide that California's OPR plans to issue in 2020 (Governor's Office of Planning and Research 2019). In the meantime, OPR offers communities a curated online repository of energy storage resources. In their 2017 Final Report on Policy Recommendations and Guidelines for Permitting Energy Storage prepared for the California Energy Commission, various stakeholders recommended the use of a standardized, streamlined, and expedited energy storage permitting processes across California jurisdictions largely based on existing small-scale PV system permitting processes, plus additional training for contractors (Sovereign Energy and Clean Coalition 2017).

Like California, New York has been a first mover in the establishment of storage permitting best practices. For example, in April 2018 the New York State Energy Research and Development Authority, in partnership with the Smart Distributed Generation Hub, ConEdison, and various New York City departments, published the Energy Storage System Permitting and Interconnection Process Guide For New York City Lithium-Ion Outdoor Systems, outlining how different sizes of outdoor storage systems should be treated in the unique, dense, urban environment of New York City. ${ }^{59}$ The New York State Energy Research and Development Authority also plans to issue guidance on storage siting outside NYC and in indoor settings (McAllister et al. 2019). In both California and New York, the development of local permitting best practices, guidelines, and resources for battery energy storage systems has been and continues to be a multi-stakeholder-driven process that considers fire, electrical, and building codes and standards.

As penetrations of DPV-plus-storage systems increase, the need for clear, standardized guidance pertaining to battery safety issues becomes more pressing. Equipment requirements apply to all aspects of energy storage systems, from the design and construction of small parts of the energy storage system (e.g. wires, switches) to how system components (e.g. inverters) work together, and codes dictate how systems are integrated into the built environment (Conover 2014). Many storage technologies, particularly those with high temperature or energetic chemistries (e.g., lithium-ion batteries), have spatial sensitivity (where they are allowed to be installed) and temperature sensitivities where cooling or heating may be required so they perform safely during all times and during all seasons (Akhil et al. 2013; Byrne n.d.). Particularly in the case of batteries, these new installations can pose fire hazards for system owners, first responders, utility employees, and system installers.

Ongoing NREL-led research tested battery performance characteristics under different environmental conditions using NREL's Energy System Integration Facility's testing chambers. Results indicate that lithium-ion batteries de-rate significantly at high temperatures and shut down entirely upon reaching $115^{\circ} \mathrm{F} / 46^{\circ} \mathrm{C}$, as aligned with relevant equipment standards. This temperature-induced shutdown reduces safety concerns (e.g., fires, explosions) due to high temperatures, but does demonstrate that temperature can impose operational constraints that utilities and customers should be aware of from both technical and economic perspectives.

Safety codes and regulations can help ensure DPV-plus-storage systems will perform as expected, regardless of environmental conditions, and not degrade due to improper installation, operation, or transportation. Building and fire codes, like those adopted in California (see the text box below) and New York (as previously discussed), address indoor and outdoor storage systems' safety concerns separately given different environmental conditions and risks. Important features called out in building and fire codes include spacing, security (e.g., fencing for outdoor systems), placement of electrical disconnects and emergency stops, construction building or encasement material (e.g., noncombustible), fire suppression systems, and ventilation and exhaust systems. Appendix C includes some major standards and codes in the United States, covering the testing, performance, and installation of DPV-plus-storage systems installed behind-the-meter, but the list is not exhaustive. Policymakers in their respective countries can assess if and how their safety codes address behind-the-meter battery storage and what additions, or adjustments may be needed for this technology.

\footnotetext{
${ }^{59}$ It should be noted that this guide only offers guidance for outdoor systems, due to the New York City Fire Department's concern about siting lithium-ion battery systems indoors.
} 


\section{Fire Codes and Market Implications for Storage-Plus-DPV Systems}

Sometimes codes and standards must be adapted to cater to the characteristics of emerging energy technologies, as in the case of behind-the-meter lithium-ion batteries. When governments are slow to adapt, this can create confusion for developers and impede deployment until stakeholders have time to understand and adapt to new rules. For example, the California Building Standards Commission adopted a state fire code revision in July 2018 that places more stringent spacing and fire suppression requirements on indoor and outdoor lithium-ion batteries $20 \mathrm{kWh}$ or greater (previously $250 \mathrm{kWh}$ or greater) (GTM 2018). This new requirement is adopted from the recently released National Fire Protection Association (NFPA) 855 "Standard for the Installation of Stationary Energy Storage Systems" that will go into effect in 2020 (Hyde 2018). These new regulations have caused concerns and project delays from developers and led some market analysts to decrease their 2019 and 2020 nonresidential battery deployment forecasts for California (GTM 2018). 


\section{Policy and Market Context for DPV-Plus-Storage}

\section{$\underline{\text { Key Insight for Indian Stakeholders }}$}

This section focuses on how the broader policy and market context for DPV-plus-storage systems can impact how regulation is developed and promulgated. The section is divided into several subsections which highlight different aspects of specific policies and market attributes that may influence DPV-plus-Storage deployment and operation. These policy and market efforts have either been developed explicitly to incentivize the deployment of storage or DPV-plus-storage systems or have indirectly incentivized such hybrid systems relative to standalone DPV systems by rewarding behavior of which only the former is capable.

India features Renewable Purchase Obligations which deem rooftop solar as eligible resources (MNRE 2018), nationally mandated state-specific targets for rooftop solar (MNRE 2019), tax incentives for domestic photovoltaic module manufacturers (KPMG 2015), and tax incentives for rooftop solar systems offered by the national government and in some instances state governments. As of the time of this writing, the central government is in the process of designing tax incentives for lithium-ion battery manufacturing. However, no incentive schemes or other major policies to support battery deployment - whether at the utility scale or behindthe-meter - are currently offered in India. Thus, this chapter discusses a variety of policy options for consideration by Indian policy makers at the state and central government level.

One possible approach for Indian policy makers to consider would be the extension of existing investment-based financial incentives for rooftop solar systems to include coupled behind-the-meter storage systems. The application of this incentive could be uniform across each state, or offered in a customized manner to reflect different system conditions (e.g., increased incentives for states with regular grid outages). Indian regulators can also consider encouraging or mandating DISCOMs in their jurisdictions to explore opportunities to use storage and other distributed assets to reduce operating costs or provide system services. As has been seen in the United States, distribution system operators may find that DER including DPV-plus-storage systems can be used to offset the need for more expensive traditional transmission and distribution system upgrades. Furthermore, some leading utilities have managed to work with customers to share behind-the-meter assets to the mutual benefit of both parties. These programs have allowed DISCOMs to utilize such assets when they would otherwise sit idle and have meant that both public (DISCOM) and private (customer) capital investments were used to install and operate the systems, which could be particularly beneficial for cash-constrained Indian DISCOMS.

Regulatory decisions are made in the broader context of a policy and market environment. This policy and market context influences all other steps in the decision-making process. The following section provides a brief overview of various policy, market instruments, and approaches that may have relevance for DPV-plus-storage systems.

These include, among others:

- Storage Deployment Mandates (Section 8.1)

- Nonbinding Storage Goals (Section 8.2)

- Renewable Portfolio Standards and Clean Peak Standards (Section 8.3)

- Direct Financial Incentives (Section 8.4)

- Distribution Network Service Provision (Section 8.5)

- Wholesale Market Participation (Section 8.6)

- Distribution Market Structure

- Distribution Company Performance Standards 
The latter two points are beyond the scope of this report, but have important implications for financing, investment, ownership, and operation and maintenance of DPV-plus-storage projects, as well as the fostering of competitive markets where new service providers have access alongside incumbent distribution utilities.

\subsection{Storage Deployment Mandates}

Governments can directly require utilities to procure specific levels of behind-the-meter storage capacity using deployment mandates. Most U.S. storage-related mandates to date have been stated in terms of total power capacity $(\mathrm{kW})$ or energy capacity $(\mathrm{kWh})$. Utilities may be subject to a single target or a series of consecutive, graduated targets over time.

For ensuring desired outcomes with storage-only or DPV-plus-storage deployment mandates, governments can consider specifying who is allowed to own eligible behind-the-meter storage systems. Should utilities be allowed to own these customer-sited assets, or can eligible third parties and/or customers be allowed to own them as well? Regulatory guidance to utilities on acceptable procurement mechanisms for behind-the-meter storage can help to reduce regulatory uncertainty and ensure utility buy-in for the mandate.

\section{Jurisdiction Examples}

California-In October 2013, in response to Assembly Bill 2514, the California Public Utility Commission issued Decision 13-10-040, which mandated their three investor-owned utilities (IOUs) to procure 1,325 MW of storage by 2020 (CPUC 2013). The bill also created interim, graduated storage mandate targets for each of the IOUs for 2014, 2016, and 2018. Furthermore, the storage mandate targets were divided between three different "grid domains," or points of interconnection (transmission, distribution, and behind-the-meter), with $200 \mathrm{MW}$ of storage required to be behind-the-meter.

The California IOUs are allowed to defer up to $80 \%$ of their required storage capacity to later procurement periods and are allowed to shift up to $80 \%$ of their required storage capacity between the transmission and distribution grid domains, although no target shifting is allowed for customer-sited storage. This flexibility and graduated targets should allow the IOUs to align the storage mandates with their actual territories needs and to make the most economically efficient investments. The IOUs are furthermore allowed to own up to $50 \%$ of all the storage capacity across all three grid domains, including behind-the-meter systems (CPUC 2013).

For ensuring utility compliance with the deployment mandate, California utilities can use a range of mechanisms, including: (1) count customers for compliance who installed storage systems independently in response to existing incentive programs and compensation mechanisms; (2) create regulated utility programs that involve direct utility ownership of customer-sited storage systems; (3) create regulated utility programs which involve private ownership of customer-sited storage systems, where financial incentives are offered to grant the utility control over the storage system; and (4) conduct a competitive procurement for grid services from aggregated customer-sited storage systems to which third parties and aggregators can respond.

\subsection{Nonbinding Storage Goals}

In addition to setting binding deployment mandates, jurisdictional authorities can also set aspirational or nonbinding goals for utilities to reach. Instead of including punitive measures to incentivize procurement, policymakers can attempt to remove barriers to deployment or provide additional funding. Some U.S. states have instituted nonbinding storage goals.

\section{Jurisdiction Examples}

New York-In 2018, the governor of New York established a nonbinding goal of 1,500 MW of storage by 2025 and 3,000 MW by 2030. The goal directs New York's relevant regulatory bodies to remove barriers to the adoption of storage and signals a strong future for storage to potential developers. The target does not differentiate between stand-alone storage or PV-paired storage, or larger front-of-the-meter installations and behind-the-meter installations. While not directly incentivizing DPV-plus-storage systems, many of the changes to policy and regulations which may stem from the target, such as streamlining interconnection processes or fire-safety approvals may nevertheless be valuable for encouraging such installations (NYPSC 2018a). 
In addition to the target, the New York Independent System Operator (NYISO) explicitly allows the aggregation of behind-the-meter batteries to facilitate their participation in the wholesale energy market, which could open up an important revenue stream for DPV-plus-storage systems in the near future, and allows for dual participation of such systems in retail and wholesale markets (NYISO 2017).

Massachusetts - In 2017, the Department of Energy Resources adopted an aspirational target of $200 \mathrm{MWh}$ for distribution companies to procure by 2020. As with New York, this aspirational goal fits into a larger plan, the Energy Storage Initiative, which provides funding for rebates and studies, and recommends regulatory changes to help distribution companies achieve the targets (DOER 2017).

\subsection{Renewable Portfolio Standards and Clean Peak Standards}

Renewable portfolio standards (RPS) are obligations regulators impose on utilities that require a certain level of energy used to meet demand or a certain level of generating capacity in a utility territory come from qualifying resources. Clean Peak Standards (CPS) are a relatively new variation on RPS and mandate that a certain portion of peak demand or net peak demand ${ }^{60}$ be met using renewable energy sources. These policies reflect a growing interest by policymakers and regulators to reduce the magnitude of peak demand and/or supply clean electricity to meet peak demand, as opposed to using conventional peaking capacity (e.g., open cycle gas turbines) that is typically expensive and less clean to operate.

In RPS or CPS schemes, a trading platform or registry creates, issues, and finally retires tradable certificates, which represent the renewable or clean aspect of a MWh of generation from qualifying resources. Regulators use these certificates to track compliance with the RPS or CPS. These certificates are awarded to eligible generators who can then sell them to complying entities, such as distribution utilities, who in turn use the certificates to meet their RPS or CPS obligations, which are determined by regulators. When a utility surrenders their acquired certificates, the certificates are retired to prevent the same certificate from satisfying the requirements of multiple power purchasers.

Under an RPS, qualifying generators are awarded renewable energy certificates for any generation that is injected to the grid. Under a CPS, qualifying generators are awarded clean peak certificates for any generation exported to the grid during a CPS compliance window. This compliance window is set well in advance of the initiation of the CPS program, usually concurrently with the establishment of the annual minimum generation percentage target.

While these policy tools are generally focused on incentivizing the deployment of utility-scale renewable generation assets, they can be adjusted to also include distributed assets and storage. RPS have already been expanded to incentivize DG deployment through the use of special carve-outs in the RPS or credit multipliers for DG resources, and it is plausible these elements in an RPS or CPS could be similarly used to incentivize storage and DPV-plus-storage deployment (Donalds 2017; Holt and Olinksy-Paul 2016). While credit multipliers could be used to incentivize the pairing of DPV and storage, they also could have the effect of lowering the overall level of desired clean energy capacity that is procured to satisfy the RPS or CPS, and thus should be considered carefully.

Even without the presence of credit multipliers or carve-outs, a CPS could incentivize the pairing of solar and storage by creating an additional value stream for storage systems. This is particularly true if the CPS compliance window occurs outside solar generation hours and can be used for either distributed or centralized systems. Under a CPS, storage could help DPV systems qualify for certificates by shifting renewable energy generation to meet peak demand, and net peak demand in particular (Burgess and Olinsky-Paul 2018).

As storage cannot generate electricity in and of itself, it is important to prevent accounting errors (e.g., double counting) of qualifying energy generation to ensure REC and CPS integrity. Generally, there are three cases when ensuring renewable energy certificate and CPS integrity: (1) the storage can charge from multiple sources, and the generation into the storage system is known; (2) the storage can charge from multiple sources, but the generation

\footnotetext{
${ }^{60}$ Net demand can be defined as the total electricity demand in a power system minus variable renewable energy generation. It represents the electricity demand that must be met by non-variable renewable energy resources, such as fossil-fuel resources, hydropower or nuclear energy. Net peak demand is the maximum value that net demand exhibits in a given period.
} 
into the storage system is not known; and (3) the storage can only charge from an eligible generating source. In the first case certificates can be issued based on the generation mix used to charge the storage system. In the second case, certificates may only be issued to the generating resources, which would not incentivize storage. In the third case, certificates can be issued based on the generator or storage output. When certificates are based on the generator, this would ignore round-trip losses in the storage system; however, when certificates are based on storage output, the generator would be effectively taxed for those losses even though the grid may benefit from the use of storage to shift generation (Holt and Olinksy-Paul 2016).

\section{Jurisdiction Examples}

Massachusetts-In July 2018, the Massachusetts House passed the Act to Advance Clean Energy, which required the Department of Energy Resources to establish a CPS and a minimum share of energy sales to customers, which must come from clean peak resources (defined as a qualified clean energy resource, storage system, or demand response resource that generates or discharges energy to the power system, or reduces load, during a seasonal system peak period). This minimum share of energy sales would increase by $0.25 \%$ per year thereafter (Golden et al. 2018). According to draft regulations, storage is qualified for accreditation under the CPS as long as it can prove that it operates primarily to store and discharge renewable energy through one of the following conditions (Baker et al. 2019):

1) The storage system is collocated with a renewable generation resource;

2) The storage system is contractually bound with a noncollocated renewable generation resource; or

3) The storage system charges coincident with designated Qualified Energy Storage System charging periods, shown in Table 4. 


\section{Table 4. Qualified Energy Storage System Charging Windows by Renewable Energy Source}

\begin{tabular}{|l|l|l|}
\hline & Energy Storage Charging Windows \\
\hline Clean Peak Season & Solar-Based Charging Hours & Wind-Based Charging Hours \\
\hline Winter & 10 a.m.-3 p.m. & 12 a.m.-6 a.m. \\
\hline Spring & 8 a.m.-4 p.m. & 12 a.m.-6 a.m. \\
\hline Summer & 7 a.m.-2 p.m. & 12 a.m.-6 a.m. \\
\hline Fall & 9 a.m.-3 p.m. & 12 a.m.-6 a.m. \\
\hline
\end{tabular}

Adapted from: Baker et al. 2019

For Massachusetts, the Clean Peak compliance window will be redetermined at least every 5 years based on changes in the state's load shape, but has been determined to coincide with net peak demand. The compliance window changes for each season and will initially be: Spring, March 1-March 14 from 5 p.m. to 9 p.m.; Summer, May 15-September 14 from 3 p.m. to 7 p.m.; Fall, September 15-November 30 from 4 p.m. to 8 p.m.; Winter, December 1-February 28 from 4 p.m. to 8 p.m.

Arizona-In October 2018, a commissioner for the Arizona public utility commission introduced an Energy Modernization Plan, which set forth several targets, including $80 \%$ clean energy by 2050 , a 3-GW storage mandate by 2030 , rules for promoting electric vehicle growth, and a CPS starting at $1.5 \%$ and increasing by $1.5 \%$ every year thereafter (Tobin 2018). The plan has not yet been finalized as of the time of this report's writing.

Washington-In 2013, the Washington legislature introduced a bill, which ultimately was not adopted, to modify the state's RPS to include credit multipliers for qualifying generating systems combined with energy storage. The bill would have allowed utilities to count electricity from energy storage facilities at 2.5 times the normal rate if the facility was "capable of storing energy from an eligible renewable resource during off-peak hours and dispatching the energy as electricity to an electrical transmission or distribution system during peak hours" (Morris et al. 2013, 6).

\subsection{Direct Financial Incentives}

In addition to changes to tariff structures or mandates, policymakers can create financial incentives, which can help customers meet the upfront capital costs involved in purchasing DPV-plus-storage systems and improve their return on investment. Financial incentives are either direct (i.e., cash) or tax-based. Tax-based incentives can be production-based (i.e., tax credit for every kWh of renewable energy produced), investment-based (e.g., tax credit equal to a fixed percentage of eligible project costs), or tax exemptions (i.e., eliminate or reduce a specific tax). Tax credits reduce the overall lifetime cost of a DPV-plus-storage project by reducing the taxes that the owner of a project owes its government.

Direct financial incentives are typically either upfront cash incentives or production-based cash incentives. Upfront rebates reduce the initial cost of installing a DPV-plus-storage system while performance-based rebates provide ongoing payments to a system owner based on the system's actual $\mathrm{kWh}$ production. Upfront rebates are typically expressed on a per-watt basis (e.g., \$1.00/watt). The value of these incentives tends to fall over time when certain installed capacity targets are reached or in parallel with declines in market costs for DER projects. Participation rebate programs can be limited to systems which share certain characteristics (e.g., system size limits, technical configurations) or which agree to certain behaviors (e.g., data sharing, self-consumption only). 


\section{Jurisdiction Examples}

California-California's Self-Generation Incentive Program ${ }^{61}$ provides rebates for qualifying energy systems, including battery energy storage systems. To be eligible for the funds, qualifying systems must: (1) be connected to the local utility's distribution system on the customer's side of the meter; (2) be configured to run in parallel with the grid; (3) be a permanent installation for the useful life of the system; (4) be capable of discharging at rated capacity for a minimum of 2 hours; and (5) discharge fully at least 52 times per year. Of the total program budget, $75 \%$ is reserved for storage with the rest devoted to generation technologies; furthermore, $15 \%$ is reserved for projects with a capacity less than or equal to $10 \mathrm{~kW}$. The program consists of a series of declining incentive rates $(\$ / \mathrm{kWh})$ for storage systems and allots a maximum budget to each incentive rate. When the budget for the largest incentive rate is exhausted, the succeeding systems are offered the next largest incentive rate. Thus the limited 'budget bins' act as a spending cap for the entire rebate program. Table 5 shows how the allotted budget for each eligible storage type changes as the program progresses through the steps when the previous step's budget is exhausted. It also outlines the step-down in incentives for each step. Although the budget is allotted on a lottery basis, priority is given to projects which would have additional grid benefits or reduce greenhouse gas emissions (CPUC 2017a).

Table 5. California Small Generator Incentive Program Budget and Incentive Levels

\begin{tabular}{|c|c|c|c|c|c|c|}
\hline & & Step 1 & Step 2 & Step 3 & Step 4 & Step 5 \\
\hline \multirow{2}{*}{$\begin{array}{l}\text { Large Storage } \\
(>10 \mathrm{~kW})\end{array}$} & Budget* & $\$ 42,728,094$ & $\$ 102,959,451$ & $\$ 75,890,956$ & $\$ 75,890,956$ & $\$ 32,046,071$ \\
\hline & Incentive & $0.50(\$ / W h)$ & 0.40 (\$/Wh) & 0.35 (\$/Wh) & 0.30 (\$/Wh) & $0.25(\$ / W h)$ \\
\hline \multirow{2}{*}{$\begin{array}{l}\text { Large Storage } \\
\text { Claiming ITC } \\
(>10 \mathrm{~kW})\end{array}$} & Budget* & $\$ 42,728,094$ & $\$ 102,959,451$ & $\$ 75,890,956$ & $\$ 75,890,956$ & $\$ 32,046,071$ \\
\hline & Incentive & $0.36(\$ / \mathrm{Wh})$ & $0.29(\$ / \mathrm{Wh})$ & $0.25(\$ / W h)$ & $0.22(\$ / W h)$ & $0.18(\$ / W h)$ \\
\hline \multirow{2}{*}{$\begin{array}{l}\text { Residential } \\
\text { Storage } \\
(\leq 10 \mathrm{~kW})\end{array}$} & Budget & $\$ 7,540,252$ & $\$ 14,232,625$ & $\$ 10,526,843$ & $\$ 10,526,843$ & $\$ 5,655,189$ \\
\hline & Incentive & 0.50 (\$/Wh) & 0.40 (\$/Wh) & 0.35 (\$/Wh) & 0.30 (\$/Wh) & 0.25 (\$/Wh) \\
\hline
\end{tabular}

Adapted from: (CPUC 2019a; SCE et al. 2017)

Arizona-Starting in May 2018, Arizona utility Salt River Project began offering a \$150/kWh-DC rebate, up to $\$ 1,800$ for residential customers who installed a qualifying battery storage system and agreed to participate in their battery study. The program is available for three years or up to a program cap of 4,500 customers if they choose to participate. Only lithium-ion batteries certified to UL 1973 or UL 9540 are eligible. The study will focus on how customers use battery systems, how battery systems perform in Salt River Project's environment, and how residential battery systems affect the operation of the grid (SRP 2018).

Arizona Public Service also announced a Storage Rewards program in September 2018 that provides residential customers a one-time $\$ 500$ bill credit for allowing Arizona Public Service to site a behind-the-meter storage system in their residence and grant APS control over the asset. The pilot program will help Arizona Public Service understand how behind-the-meter storage can support peak demand management (APS 2018c).

Massachusetts-As part of its Energy Storage Initiative, the Department of Energy Resources (DOER) has suggested the implementation of a rebate, Massachusetts Offers Rebates for Storage Program, known as "MORStorage," for behind-the-meter storage projects at commercial and industrial businesses. The \$20 million grant will be available for both stand-alone storage and for storage paired with on-site solar PV generation. In addition

${ }^{61} \mathrm{http}: / /$ www.cpuc.ca.gov/sgip/ 
to requirements on cost sharing, eligible projects must be able to demonstrate that the storage is providing a service that benefits all consumers, such as reducing on-site peak demand and that the project is capable of dispatching in response to local distribution system needs (Customized Energy Solutions et al. 2017).

In addition to rebates for select projects, Massachusetts also provides a financial incentive for DPV-plus-storage systems through the Solar Massachusetts Renewable Target (SMART) program. ${ }^{62}$ Through this program, DPV owners receive payments for solar energy produced at a fixed $\$ / \mathrm{kWh}$ rate. Systems can also qualify for NEM credits, and the total compensation amount is the difference between the original SMART compensation and the NEM compensation to prevent double-counting. The SMART incentive rate is based on a declining block design wherein customers receive a set compensation rate up until a program cap is reached, after which the compensation rate is reduced for new applicants. In September 2018, the DOER clarified the eligibility of storage systems to participate in the SMART program. Storage systems co-located with solar installations which meet requirements on minimum efficiency, minimum/maximum nominal rated power, minimum/maximum nominal useful energy, and data provision can qualify for an Energy Storage Adder to the existing SMART rate, which applies to the level of solar generation of the paired system. Behind-the-meter installations which can meet the physical and operational requirements can also qualify (DOER 2018).

Nevada-In response to Senate Bill 145, NV Energy released an energy storage incentive program in September 2018. Customer eligibility, system size requirements, and incentive levels (\$/watt-hour) vary by customer category (i.e., residential, small commercial, large commercial, low income/nonprofit, public entity). All eligible systems must be capable of being charged $75 \%$ by renewable energy sources. Customers on TOU rates receive a higher maximum incentive rate than non-TOU customers; similarly, commercial customers in a "critical infrastructure" area receive a higher rate than those in a noncritical infrastructure area. For all customer categories, incentive levels "step down" to a lower rate each time \$1 million USD in incentives has been disbursed, with $\$ 5$ million reserved for program funding across all customer categories (NV Energy 2018b). Thus, as with California's SGIP, the allotted budget functions as a program cap to ensure cost containment.

\subsection{Distribution Network Service Provision}

Utility DER-aggregation programs and pilots are typically intended to provide load control and demand response services to the local distribution system, as opposed to the wholesale market (Cook et al. 2018). In these more common use cases, distributed resources are either aggregated directly by the utility or by third parties, which then interface with the utility as a single resource to provide valuable services to the distribution system, such as congestion management. Similar to aggregated resources participating in wholesale energy markets, these resources must have the ability to receive and respond to signals, as well as relay their operational decision to respond back to the utility or third-party aggregator.

In addition to technical barriers to optimizing battery operations for the provision of multiple services, such as immature communication or battery control technologies, there can also be market and policy barriers to this kind of value stacking for stand-alone battery storage and DPV-plus-storage systems. Several of these potential market and policy issues are highlighted in the Market and Policy Barriers to Value Stacking text box.

\footnotetext{
${ }^{62}$ For additional information on Massachusetts' initiative, visit:
} 


\section{Market and Policy Barriers to Value Stacking}

In addition to the technical barriers to value stacking, there are several market and policy barriers which may prevent distributed storage-plus-DPV systems from providing multiple system services. First, in order to profit from providing a system service, these services must be valued either through markets or other mechanisms. Currently in many power systems, services such as governor response or black-start are not valued, and are instead required as a prerequisite to interconnection without opportunity for compensation. Second, even if system services are compensated, batteries and other distributed resources may not be fairly considered for service provision as they are relatively new and utilities are unfamiliar with the technologies. Finally, in some jurisdictions, systems may be barred from receiving compensation from both wholesale markets (for services like frequency regulation) and cost-of-service arrangements (Bhatnagar 2013). Regulators have an important role in clarifying that DERs are allowed to provide certain system services and ensuring that they are both adequately compensated for services provided as well as fairly considered for service provision in the first place. These considerations must be balanced with utility concerns to ensure that the provision of certain services from batteries and storage-plus-DPV systems does not compromise the safe, reliable operation of the power system.

\section{Jurisdiction Examples}

Hawaii-In 2014, as a part of DOE- and U.S. Navy-funded Energy Excelerator program, the utility (Hawaiian Electric) and battery operator (Stem) began installing behind-the-meter storage and storage-control systems. These systems were located on 29 commercial sites on the island of O'ahu, reaching a total installed capacity of $1 \mathrm{MW}$ by 2017. Using advanced software control, weather forecasts, and historical and real-time load data, these storage systems can be deployed to help commercial entities reduce peak demand usage, leading to larger bill savings for customers. The storage controls also allow Hawaiian Electric to aggregate these systems into 'virtual power plants.' The utility can call upon the virtual power plants in order to manage variations in solar PV output or other disturbances on the distribution system, allowing Hawaii to integrate increasing levels of renewable energy resources (HECO 2017; 2015b). Although all of these projects were stand-alone storage units, with advanced system controls, the same principle could be extended to DPV-plus-storage systems.

New York-As a part of the Reforming the Energy Vision initiative, the New York Public Service Commission directed its major IOUs to create demonstration projects which would "inform regulatory changes, rate design, and the most effective means to integrate DER on a larger scale" (NYPSC 2015, 118). ${ }^{63}$ In response to this directive, the utility New York State Electric and Gas proposed and began implementing its "Aggregated Behind the Meter Energy Storage" demonstration project, which seeks to "demonstrate some of the value streams that can be leveraged in parallel by behind-the-meter battery storage and attempt to identify new value streams ... [and] evaluate potential alternative rate designs and their impact on the value proposition of aggregated behind-themeter battery storage" (NYSEG 2018, 3). Among other goals, the demonstration project seeks to prove that commercial and industrial customer storage systems can aggregate into a virtual power plant to reduce distribution circuit and system peaks. The demonstration project will install a mixture of small to larger battery systems at eight customer sites for a total of $1.06 \mathrm{MW}$ and $4.2 \mathrm{MWh}$ of battery storage capacity. By 2021 the utility hopes to be able to show, through data from metering equipment installed as part of the project, that the systems were able to reduce circuit peak during times of system-wide peak. Actual targets for circuit peak reduction will be determined once the customers have been chosen and the systems installed.

Vermont - Through its Resilient Home program, utility Green Mountain Power in Vermont offers its customers Tesla Powerwall batteries for $\$ 15 /$ month, which customers can use to store excess solar power or to provide backup power during outages, in exchange for the ability to discharge the batteries to reduce its distribution system's peak demand. Customers can also enroll in the program without leasing the battery pack through the utility's Bring Your Own Device program, where they receive a fixed monthly bill credit from Green Mountain Power for their participation. ${ }^{64}$ As of August 2018, the utility had more than 600 customers in the program and

${ }^{63}$ All demonstration projects can be found at: https://rev.ny.gov/rev-demo-projects-1

${ }^{64} \mathrm{https}$ :/greenmountainpower.com/product/powerwall/. 
used the distributed solar-plus-storage systems to reduce demand during the summer peak periods, saving customers an estimated $\$ 500,000$ in 2017 and $\$ 600,000$ in $2018{ }^{65}$

\subsection{Wholesale Market Participation}

One large potential source of income for utility-scale battery storage systems is the provision of peaking capacity and ancillary services in wholesale electricity markets (Denholm 2019). Regulatory barriers to utility-scale storage systems in U.S. markets are being addressed with implementation of FERC Order 841, which stipulated that regional transmission organizations and independent system operators must revise their tariffs to allow for a 'storage participation model' that would facilitate storage's entry into the regional transmission organizations/independent system operators markets (FERC 2018b). The FERC order explicitly declined to expand the order's applicability to behind-the-meter storage that did not inject power to the grid and set a minimum size requirement for market participation of $100 \mathrm{~kW} .{ }^{66}$

Minimum size constraints - which are intended to streamline power system operation in wholesale energy markets - prohibit most behind-the-meter storage systems and DPV-plus-storage systems from direct participation in wholesale power markets by themselves, in particular for systems sited with residential customers.

The aggregation of smaller DERs into a larger "virtual" resource can help smaller plants still participate in the wholesale market despite the minimum size constraints. In these use cases, DERs are typically aggregated by a private market participant. This aggregating entity is responsible for ensuring that smaller resources have the ability to receive signals, modify their operations as needed, and communicate their operational pattern to relevant entities.

The aggregation of DER for wholesale market provision may raise concerns over operational impacts to the distribution system, especially at the feeder level. Resources on the distribution system responding to signals to provide regulation services to the bulk-power system, for example, may increase feeder peak load or drastically impact voltage at the feeder. This is particularly true for energy storage which can both charge and discharge, potentially impacting voltage at the feeder in both directions (FERC 2018a). Despite these challenges, efforts are under way in several markets to enable smaller individual resources to be aggregated into larger systems for wholesale market participation.

\section{Jurisdiction Examples}

California-In 2016, CAISO modified its tariff structure to allow aggregated DERs, including behind-the-meter battery storage, to participate in its independent system operator market to provide both energy and ancillary services (California ISO 2016).

In 2015 PG\&E, an investor-owned utility, started the Supply Side DR Pilot, which was designed to study aggregated resources' ability to supply a proxy demand resource product in the wholesale market (Anderson and Burrows 2017; California ISO 2010). A proxy demand resource is defined as "a California Independent System Operator market product where retail customer loads are bid in to the CAISO's wholesale energy market for purposes of demand response. A PDR can be one or more retail customer accounts that have been entered as a single resource into the CAISO's demand response system by a demand response provider." Systems from five third-party aggregators successfully provided both customer-facing services (e.g., demand charge reductions) and bulk-power system services (California ISO 2010). The pilot was updated and continued in 2017 to help provide local distribution services in addition to customer-facing and bulk-power system services (PG\&E and Olivine 2017).

\footnotetext{
${ }^{65} \mathrm{https}$ //greenmountainpower.com/news/gmp-beats-new-peak-delivers-bigger-customer-savings-with-growing-network-ofstored-energy/.

${ }^{66}$ FERC argued that behind-the-meter storage systems which did not inject power into the grid were adequately covered under existing rules for demand response. The FERC order did not explicitly set the minimum size requirement to $100 \mathrm{~kW}$, but rather required RTOs and ISOs to establish their own minimum size requirement that did not exceed $100 \mathrm{~kW}$. Although FERC did not explicitly focus on behind-the-meter storage, barriers to smaller batteries could be similarly removed.
} 
Regulators in California have also pioneered the development of market rules surrounding value stacking issues for batteries. Specifically the rules (CPUC 2018a):

- Dictate that batteries can only provide services at the voltage level to which they are interconnected or higher, but not lower (i.e., transmission-level assets can't provide customer-level services)

- Develop categories of services and prioritize "reliability services" over "nonreliability services" and ensure that batteries cannot contract for additional services that would interfere with any obligation to provide "reliability services"

- Require that batteries comply with all performance and availability requirements for services it provides and that noncompliance penalties be communicated in advance

- Require that batteries inform the utility of any services it currently provides or intends to provide

- Take measures to prevent double compensation to batteries for services provided.

These rules help ensure that batteries can select the most cost-effective combinations of services to provide without negatively impacting the reliability of the grid (CPUC 2018a). Although the rules are not specifically oriented towards DPV-plus-storage systems, such rules provide a guideline for resolving issues around maximizing the revenue streams for such systems by providing both customer- and grid-facing services.

New York-In New York, the Public Service Commission directed the NYISO to allow participation of storage systems in both retail markets and wholesale markets, allowing for a broader range of potential income streams for such projects (NYPSC 2018a). Additionally, in 2016, the investor-owned utility Con Edison launched a pilot program to aggregate DPV-plus-storage installations at 300 residential customer sites to provide both lower bills and greater resiliency for customers, as well as aggregated ancillary services to be bid into the NYISO wholesale market on behalf of the utility (ConEdison 2016). The pilot was suspended in 2017 due to permitting issues for storage at residential sites with the Fire Department of New York and the NYC Department of Buildings, but Con Edison intends to resume the project once these issues have been resolved (REV Connect 2018).

FERC - With the adoption of FERC Order 841, FERC directed all wholesale energy market operators under its jurisdiction to develop a participation model for energy storage to ensure these resources can are "eligible to provide all capacity, energy, and ancillary services that the resource is technically capable of providing" (FERC 2018b). This participation model should apply to all storage resources, regardless of whether they are behind-themeter or on the distribution or transmission system, so long as they are of at least 100-kW capacity. Although the order does not create any tariffs itself, it does ensure that all wholesale markets will offer the opportunity to behind-the-meter DPV-plus-storage systems to participate in wholesale markets, so long as they can meet the minimum technical requirements for provisioning of services. While most DPV-plus-storage systems will be too small to participate, the order opens the door for widescale participation of aggregated distributed storage resources in wholesale markets.

An overview of policy and market context for DPV-plus-storage systems was covered in this section, as regulators make decisions in the broader context of a policy and market environment. This section covered policy and market instruments that may be relevant for DPV-plus-storage systems, including storage, renewable energy, and clean peak mandates, nonbinding storage goals, direct financial incentives and participation in distribution system service markets and wholesale energy markets. 


\section{Concluding Remarks: Considerations for DPV-plus- Storage Program Design}

Due to historically poor grid reliability in many Indian states, storage may add significant value to users beyond bill savings by providing backup services for customers and, potentially, valuable system services to DISCOMs. Given the familiarity of Indian consumers with storage already, proactive state-level regulatory leadershipcombined with continued battery cost declines - could result in India becoming a vibrant and quickly growing market for behind-the-meter DPV-plus-storage systems. Well-managed deployment of DPV-plus-storage could accelerate grid reliability improvements in India by reducing demand for grid electricity, especially if coupled with smart demand-side management programs to avoid grid congestion during peak times. To this end, the central government and the Indian Forum of Regulators can consider supporting SERCs and DISCOMs in modernizing retail electricity tariffs and upgrading local DER interconnection practices to accommodate gridinteractive behind-the-meter storage and DPV-plus-storage systems.

In order to achieve desired outcomes, there are a number of relevant considerations that regulators can contemplate in order to promote fair, efficient, and well-functioning programs for DPV-plus-storage resources. These considerations have been identified based on NREL's analysis of experiences in the United States, which despite differences in power system contexts, can provide important lessons for India's power system.

Determine the desired role of DPV-plus-storage. Compared to grid-tied DPV systems, DPV-plus-storage has a significantly broader set of capabilities to offer customers and the power system, and regulators are often in a position to guide how these resources are ultimately utilized. Some regulators may wish to see DPV-plus-storage systems deployed to primarily or explicitly serve a customer's own energy demand, and/or for individual customer backup purposes during grid outages. Others may wish to enable DPV-plus-storage systems to provide grid services to the power system, or simply to preserve the option to do so in the future without expensive retrofits. In any case, determining the desired role of DPV-plus-storage systems upfront and ranking them in priority order, before various rules and regulations are advanced, can help to guide a range of decisions related to compensation mechanism design, metering and technical configuration requirements, and technical interconnection processes and requirements.

Customizing rules and requirements based on the characteristics of the DPV-plus-storage system is a key strategy to promote fairness. Creating distinct sets of compensation mechanisms, metering and technical configuration requirements, and interconnection processes - which may be based on system size, the intended use of the system (e.g., an exporting versus non-exporting storage system), and other aspects - can help to ensure that regulatory requirements are appropriate for various segments of the DPV-plus-storage market.

Market context strongly influences compensation mechanism design and its timing. Solar penetration levels, grid management issues, storage deployment mandates or goals, DER technology costs, utility cost recovery and cross-subsidization considerations, and low-income customer concerns are examples of factors that may drive regulators to consider certain design choices at certain times over others. For example, the presence of higher penetrations of solar photovoltaics may create certain grid management issues (e.g., the "duck curve") that could motivate the utilization of more granular, cost-reflective rates (e.g., TOU tariffs) and/or alternatives to NEM to better align customer and grid system needs. On the other hand, jurisdictions which have financially insolvent utilities or significant cross-subsidy schemes in place may be more concerned with ensuring utility revenue sufficiency and avoiding cost-shifting, and may wish to encourage the use of customer-sited storage deployment primarily for self-supply and/or backup during grid outages. Importantly, early-stage DER markets may take different approaches to compensation mechanism design than mature DER markets, depending on these contextspecific factors and needs.

Tariff design is an important tool to align the interests of DPV-plus-storage customers with the broader power system. Relative to grid-tied DPV systems, the presence of a paired behind-the-meter storage system allows customers to better control the magnitude and timing of their electricity consumption from the grid, as well as their grid exports. TOU volumetric energy rates and coincident demand-based charges, if designed and implemented properly, can take advantage of this load shifting capability and incentivize DPV-plus-storage customers to act in a more grid-optimal manner (e.g., reducing consumption and/or increase exports during 
typical peak demand periods). This behavior, as incentivized by time-variant tariffs, can help ease the management of distributed energy resources on the distribution system and also lead to a reduction in power system operational costs. Implementation of such tariffs may require new metering equipment and administrative responsibilities for utilities but can serve as a grid-friendly incentive for customers to install DPV-plus-storage systems.

Regulators can help balance common utility concerns with consumer interests, ensuring that the implementation cost of solutions is commensurate with the scale of the issue being created. Utilities may voice concerns over issues such as compensation mechanism integrity, energy arbitrage activities, or inadvertent exports from storage systems. There are a variety of known solutions to address these issues, but many of them involve additional expenditures by customers and/or new administrative burdens for utilities. Regulators are in a position to balance utility and customer interests and ensure that utility proposals to mitigate their concerns do not place an undue burden on participating and nonparticipating customers. For instance, while undesirable energy arbitrage could be mitigated through the deployment of additional metering equipment, the cost of installing additional metering equipment might be inappropriately high for smaller scale residential, commercial, or industrial customers.

Regulators can enable business model innovation for DPV-plus-storage systems. Regulators can play a key role in allowing alternative investment and ownership models for DPV-plus-storage systems. Many U.S. DPV assets have been deployed under third-party ownership models (either lease or power purchase agreement) whereby a utility customer does not actually own the asset but receives a portion of the benefit of the DPV generation. Third-party ownership models are already beginning to play a role in storage deployment in the United States, Australia, and elsewhere, both for retrofits and new-build DPV-plus-storage systems. In the future, these resources might be aggregated by third parties, who are allowed to do so, to provide valuable services to distribution companies and/or the bulk power system. Regulators can play a key role in enabling the participation of aggregators and in some cases, defining how they are allowed to operate. This consideration links to broader questions of market reform in the distribution sector.

Standardizing interconnection application forms, processes, and requirements may offer a variety of benefits. Doing so can reduce barriers to market entry for developers, as developers would only have to learn a single process for interconnecting prospective customers with utilities, as opposed to a separate process for all utilities in a particular market. Standardization may also reduce the number of incomplete or incorrectly completed applications that utilities have to process, which both streamlines interconnection processes and reduces utility administrative burdens. On the other hand, creating standardized interconnection standards could be an additional burden for regulators to undertake the effort and periodically update it. Furthermore, additional regulatory approvals may be required if specific utilities want to modify their standards to be more specifically catered to their situation. 


\section{Appendix A-Energy Storage Technology Ecosystem}

Energy storage systems are technologies capable of charging energy from an external source and discharging this energy at a later time, after some amount of the initial energy is lost. Energy storage systems can be broadly categorized based on: (1) the type of energy they store (e.g., thermal, mechanical, electrical, or electrochemical energy); or (2) where they are interconnected (e.g., in front-of-the-meter, behind-the-meter, or off-grid). These are distinct from demand response initiatives, which seek to shift energy consumption to better match the supply of cheap or clean electricity, but do not directly store energy for later use.

Mechanical energy storage systems, such as pumped storage hydropower (PSH), compressed air energy storage, and flywheels, have historically been the most common forms of energy storage around the world, in particular PSH. PSH uses electricity to pump water from a lower-elevation reservoir to a higher-elevation reservoir and then releases the water from the high reservoir to turn hydroelectric turbines to generate electricity at a later time. Compressed air energy storage systems use electricity to store air in a reservoir, either in underground caverns or in aboveground containers. The compressed air can then later be directed through a turbine to generate electricity. Flywheels use electricity to accelerate a rotor in a very low friction environment and electricity can be extracted from the flywheel by decelerating the rotor. This technology suite is typically utilized in larger scale front-of-themeter projects, with the exception of flywheels, which may be used behind-the-meter in certain critical infrastructure settings (e.g., hospitals) such as an uninterruptible power supply (UPS) ${ }^{67}$.

Electrochemical storage systems use a series of reversible chemical reactions to store electricity in the form of chemical energy. Batteries are the most common form of electrochemical storage and have been deployed in power systems in both front-of-the-meter and behind-the-meter applications. Various battery chemistries are explained in the subsequent section.

Electrical energy storage systems store electricity either in the electric field of a supercapacitor or in the magnetic field of a superconductor for extremely short durations. At a high level, most electrical energy storage systems are capable of holding charges for long periods of time but can only discharge at their full capacity for very short durations (e.g., seconds or minutes). In power systems, supercapacitors are typically deployed as a UPS, in combination with other storage technologies such as batteries to improve the response and operation of the storage system or to help buffer fluctuations in power supply or demand. Similar to supercapacitors, superconducting magnetic energy storage is typically used in quick-response, very short duration applications, such as ensuring power quality. These storage systems are relatively new and have seen limited deployment in the power system. Furthermore, given their price and short durations they are not expected to play a large role in behind-the-meter settings except for isolated commercial and industrial customers with stringent power quality needs.

Thermal energy storage systems work either by raising or lowering the temperature of a material or by inducing a phase change in a material. Thermal energy can be stored in this material on timescales from hours to seasons based on the size of the system and can be used to meet heating or cooling demands or to generate electricity through the creation of steam from stored heat. It can be implemented using a range of technologies and approaches. For electricity generation, this technology suite is typically utilized in larger-scale front-of-the-meter projects, often in combination with generating technologies, such as with concentrated solar power plants.

Figure 1 shows several storage systems and their suitability for distributed applications.

\footnotetext{
${ }^{67}$ UPS refers to a source of backup power that is used to ensure continuous availability of power in the event of an interruption of service. UPS typically only have enough energy to operate for a few to several minutes while backup generation source or other storage systems become activated (Akhil et al. 2013).
} 


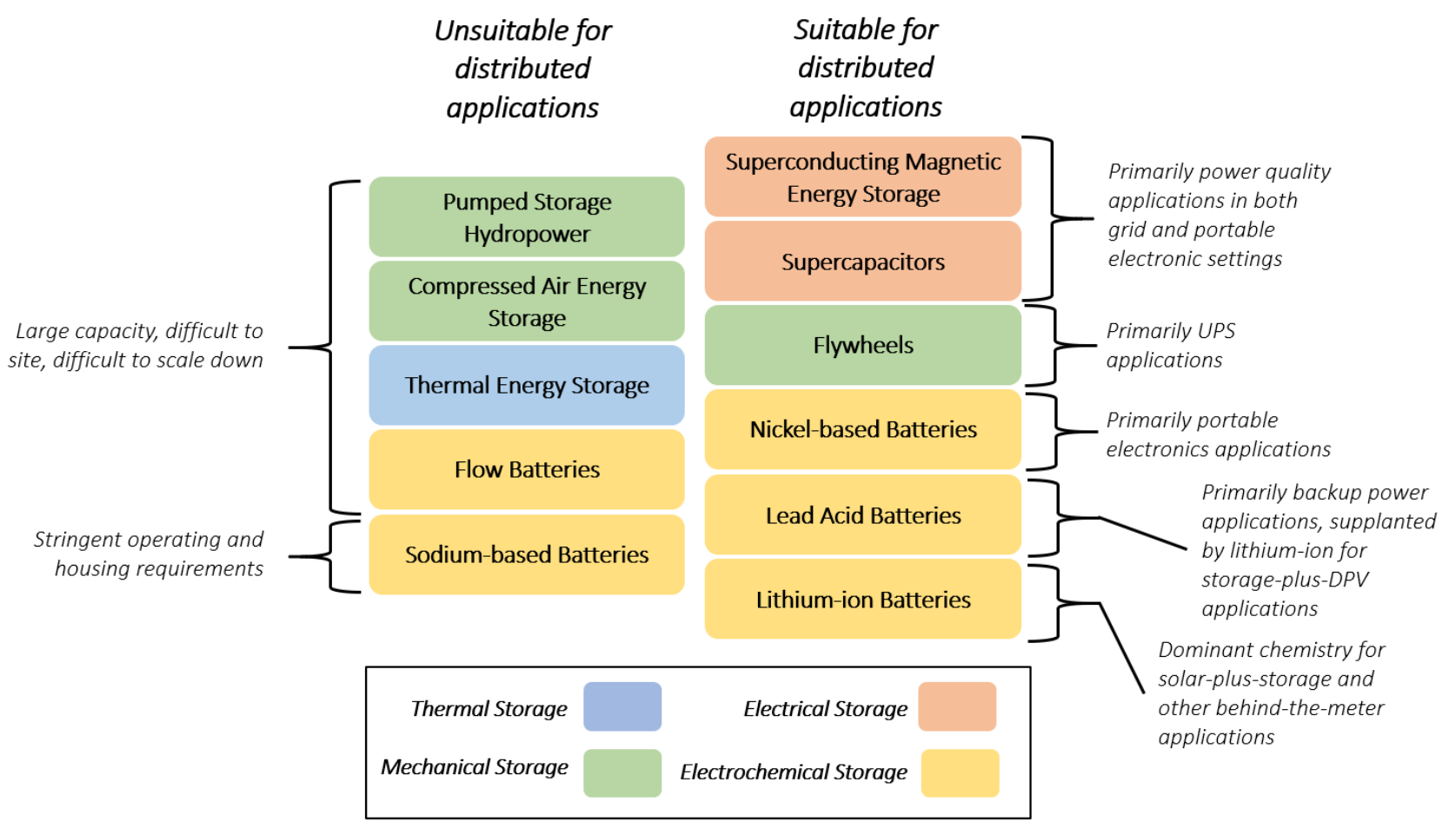

Figure 13. Storage technology types and applications organized by storage medium Source: Original [IIlustration by authors]

This figure is not intended to provide an exhaustive list of all storage technologies. Furthermore, there are a range of potential technological approaches and/or chemistries within each of the technology types illustrated. 


\section{Appendix B-Tradeoffs of Various Battery Technologies}

Lithium-ion batteries have, in recent years, become the most commonly newly installed battery technology for power sector applications, deploying in both front-of-the-meter and behind-the-meter applications. Compared to other battery technologies, lithium-ion batteries are characterized by very high energy densities, moderate power densities, a moderate cycle life, high round-trip efficiencies, moderate (and quickly declining) costs, and low maintenance requirements. The efficiency and capacity of these chemistries is not as strongly impacted by the depth-of-discharge compared to other battery chemistries, such as lead-acid or nickel-based. Lithium-ion batteries are relatively safe but have the potential for thermal runaway, in which the internal temperature of the battery rises faster than the heat can be dissipated, which can lead to fires or explosions. Despite safety concerns, lithiumion has emerged as the primary battery chemistry for behind-the-meter applications, including DPV-plus-storage systems. Careful design and appropriate safety codes can support a safer, but not foolproof, configuration. Reuse, recycling, and safe disposal of lithium-ion batteries may also be important issues for regulators.

Lead-acid batteries have seen deployment in the power sector primarily for off-grid, as well as emergency and backup power applications. They have largely been supplanted in DPV-plus-storage systems and residential customer applications by lithium-ion chemistries. Compared to other battery chemistries, lead-acid batteries exhibit low energy densities, very high-power densities, relatively poor cycle lives, moderate round-trip efficiencies, very low costs, and low maintenance requirements. Lead-acid batteries are very safe and highly reliable, which in the past has made them quite popular for UPS and backup power supply applications.

Nickel-based batteries are often used in portable applications such as power tools but have seen limited power sector deployment. These battery chemistries tend to have low energy densities, low power densities, moderate cycle lives, poor roundtrip efficiencies, moderate costs, and moderate maintenance requirements. Nickel batteries can handle wide ranges of discharging and loading and can be rapidly charged, making them one of the most durable battery chemistries. Finally, some types of nickel batteries (e.g., Nickel-Cadmium) have significant environmental concerns, given their utilization of toxic elements. These batteries also exhibit a memory effect, in which recharging the battery when it is not fully discharged permanently reduces the battery's capacity. The combination of the memory effect, poor power and energy densities, and environmental concerns has limited this chemistry's deployment in power sector applications.

Sodium-based batteries are primarily deployed for utility-scale applications in the power sector. These batteries are characterized by high energy densities, high power densities, high cycle lives, high roundtrip efficiencies, lower costs (particularly at larger scales), and low maintenance requirements, compared to other battery chemistries. These chemistries require high temperatures (in excess of $300^{\circ} \mathrm{C}$ ) to operate and have stringent housing requirements given the extremely high reactivity and corrosiveness of some of their elements. These chemistries are made from readily available, inexpensive materials which may improve their economics relative to chemistries with rare element requirements, such as some lithium-ion chemistries. Stringent housing and operating temperature requirements make this chemistry unsuitable for most behind-the-meter applications.

Flow batteries are a relatively new battery chemistry well-suited to large-scale and long-duration applications. These batteries have poor energy and power densities, very high cycle lives, low to moderate roundtrip efficiencies, moderate costs, and relatively low maintenance requirements (primarily for associated pumps). These batteries are very safe and are not prone to fires or other hazards. Furthermore, the power and energy capacities of these batteries are easily and independently tunable, making them a very flexible option from a design standpoint. Given these systems' low energy and power densities and higher relative costs, flow batteries will likely experience limited use in behind-the-meter applications in the near-term, where size constraints are more relevant. 


\title{
Appendix C-Relevant Codes and Standards
}

\author{
Rule 14H-Hawaii Interconnection of Distributed Generating Facilities with the Company's Distribution \\ System
}

Rule 14H governs the interconnection of both non-inverter-based and inverter-based generating systems located on customer premises operating in parallel to Hawaiian Electric Company's distribution system. ${ }^{68}$ The rule explicitly applies to generators that incorporate the use of an energy storage device, such as DPV-plus-storage systems. In addition to grid requirements, which are based on IEEE 1547-2003, the rule also addresses issues related to the management of the interconnection process (e.g., by providing a standard outline for interconnection agreements to be used by the utility), as well as interconnection application evaluation practices (e.g., under what scenarios the utility can initiate a supplemental technical review). Among the technical requirements outlined in the rule are transient overvoltage mitigation and advanced inverter functions that are aligned with capabilities certified by UL 1741 SA and included in IEEE 1547-2018. These functions include the ability to ride through voltage and frequency disturbances, as well as control active power output and reactive power compensation along various pre-determined functions.

Available at:

https://www.hawaiianelectric.com/documents/billing_and_payment/rates/hawaiian_electric_rules/14.pdf\#page=7

\section{Rule 21-California Generator Interconnection}

Similar to Rule $14 \mathrm{H}$, Rule 21 "describes the interconnection, operating and metering requirements for generation facilities to be connected to a utility's distribution system." ${ }^{69}$ The rule outlines technical requirements from equipment interconnecting to the power system, technical screens to evaluate systems wishing to interconnect, and parameters governing how interconnection applications must be processed by the utilities. As with Rule $14 \mathrm{H}$, Rule 21 provides required capabilities from smart inverters for inverter-based generating systems (including DPV-plus-storage systems), which can be certified using UL 1741 SA. As of the time of this writing, Rule 21 is being updated in accordance with Rulemaking 17-07-007 to incorporate power flow analysis into the technical screens in order to improve interconnection siting decisions and facilitate fast-tracking of qualifying projects (CPUC 2017b).

Available at:

https://www.cpuc.ca.gov/Rule21/

\section{UL 1741-Inverters, Converters, Controllers, and Interconnection System Equipment for Use with Distributed Energy Resources}

This equipment testing certification standard covers equipment such as smart inverters used in stand-alone or grid-connected power systems. In addition to verifying normal operating temperatures and acceptable operation during short circuit and overloading conditions, UL 1741 covers the performance of equipment as they provide specific grid functions such as low and high voltage ride through, fixed power factor and anti-islanding. The testing procedures outlined in UL 1741 are designed to certify conformance with the capabilities outlined in IEEE 1547. UL 1741 was updated to UL 1741 SA (Supplement A) to provide additional testing to ensure conformance with California's Rule 21 and Hawaii's Rule 14H for smart inverters.

Available at:

https://standardscatalog.ul.com/standards/en/standard_1741_2

\footnotetext{
${ }^{68} 14 \mathrm{H}$ is a subset of Hawaiian Electric Company's Rule No. 14, "Service Connections and Facilities on Customer's Premises."

${ }^{69}$ Each large investor-owned utility in California has its own slightly customized variation of Rule 21, which can be found on the rule's website.
} 


\section{IEEE 1547-2018 - Standard for Interconnection and Interoperability of Distributed Energy Resources with Associated Electric Power Systems Interfaces}

IEEE 1547 is a voluntary industry standard for DER connection codes that establishes criteria and requirements for interconnection of DER with electric power systems and associated interfaces (Nagarajan 2018). IEEE 1547 is technology neutral and refers to all DER connecting to the power system, including both grid-tied DPV and DPVplus-storage systems. Within the family of IEEE 1547.x standards, a new standard (1547.9) will specifically discuss considerations for behind-the-meter storage, expected in 2022. Among other technical requirements, IEEE 1547 establishes a series of performance categories that set minimum requirements for both the ability of a DER to absorb reactive power (specified under Categories A and B) and for the ability of a system to remain online during abnormal system conditions (Categories I, II and III) as well as a range of acceptable communication protocols. To meet IEEE 1547, systems and their individual components must be capable of meeting all performance categories and communication protocols. In reality, some utilities and regulators that utilize IEEE 1547 as a basis for their connection code may select which of the latent capabilities to activate according to local power system characteristics and needs. Others may choose to adopt IEEE 1547 as their connection code wholesale — without any major modifications to the various design parameters.

Available at:

https://standards.ieee.org/standard/1547-2018.html

\section{IEEE 1547.9-Guide to Using IEEE Standard 1547 for Interconnection of Energy Storage Distributed Energy Resources with Electric Power Systems}

This forthcoming recommended practice considers the specific application of energy storage systems connected at the distribution system. A separate standard was considered appropriate, given the unique characteristics of storage such as the bidirectional exchange of real and reactive power with the electrical grid. The standard will help provide guidance on technically sound approaches to interconnection and will include topics not covered in IEEE 1547, such as guidance on charging and generation constraints to minimize negative impacts to the distribution grid.

Available at: https://standards.ieee.org/project/1547 9.html

\section{NFPA 111-Standard on Stored Electrical Energy Emergency and Standby Power Systems}

NFPA 111 covers performance requirements for stored electric energy systems providing an alternate source of electrical power in buildings and facilities during interruption of the normal power source. These requirements cover, among others: power sources; transfer equipment; controls; supervisory equipment; accessory equipment needed to supply power to selected circuits; installation, maintenance, operation, and testing requirements pertaining to battery system performance. While regulators for the electric power system may not have jurisdiction over siting and permitting decisions, such as fire safety, it is important for them to take these requirements into consideration as it may impact the feasibility of developing certain DPV-plus-storage systems.

Available at:

https://www.nfpa.org/codes-and-standards/all-codes-and-standards/list-of-codes-and-standards/detail?code=111

\section{NFPA 855-Standard for the Installation of Stationary Energy Storage Systems}

Development on NFPA 855 began in 2016 in response to the growth of storage systems in the United States and was first published in September 2019. NFPA 855 covers requirements for the installation of stationary energy storage systems, such as in DPV-plus-storage systems. This includes acceptable locations for installations, sizing and separation of system components, and fire suppression and control. The standard covers electrochemical, capacitor, fuel cell, flywheel, and superconducting magnet systems. NFPA 855 also considers the ventilation, detection, signage, listings, and emergency operations associated with energy storage systems to help mitigate fire and life safety hazards. For utility-operated storage facilities, utilities in the United States are typically allowed to use the NFPA 70 and ignore NFPA 855 at their discretion. 


\section{NFPA 70-NEC}

The NEC is the de facto electrical code in the United States for the installation of electrical equipment and wiring, applying to residential, commercial, and industrial facilities. It is updated every three years, with the next version expected in late 2019. The rules cover installations, circuits and protection, methods and materials for proper wiring, and general-purpose equipment. The rules also cover special equipment, emergency systems and communication systems. The NEC also often refers to listed devices and equipment that has been properly designed, manufactured, tested or inspected by an appropriate listing agency, or national recognized testing laboratories, such as UL. Some iteration of the code has been wholesale adopted at the state-level in all but three U.S. states, with widespread county/municipality adoption of the code in those three states. The NEC has also been adopted by many U.S. territories and over a dozen countries. ${ }^{70}$ Because of the frequent changes in each update cycle, many states, cities, and counties often adopt a version of the NEC but may not adopt the latest version upon publication. Importantly, AHJs may adopt requirements beyond those in the NEC.

Available at:

https://www.nfpa.org/codes-and-standards/all-codes-and-standards/list-of-codes-and-standards/detail?code=70

\footnotetext{
${ }^{70}$ For more information on the adoption of the NEC in the United States and globally, see: https://www.nfpa.org/NEC/NECadoption-and-use.
} 


\section{Appendix D—DPV-Plus-Storage Technical Configurations}

This section provides describes several key design configurations for DPV-plus-storage systems that are popular in the United States and may become prevalent in other international contexts, and also provides illustrative diagrams ${ }^{71}$ of these configurations. These design configurations have been included primarily because they allow for backup power when energy is not available from the grid, an important consideration in both the United States and around the world. ${ }^{72}$

For DPV-plus-storage systems, one important consideration is whether to couple the hybridized system with an AC or DC connection. In AC-coupled systems, the battery and the PV array have separate inverters, while in DCcoupled systems they share a single inverter. Typically for behind-the-meter systems, DC-coupled systems share a multiport hybrid inverter that allows bidirectional power flow to and from the battery, while only allowing unidirectional power flow from the PV array. While not necessary for DC-coupled systems, this multiport hybrid inverter allows the battery to charge from both the grid and the PV array, increasing the overall flexibility of how the coupled system can operate (Ardani et al. 2017). Although a full treatment of battery and PV inverters is outside the scope of this work, the coupling strategy and associated equipment requirements of these systems may have important implications.

For behind-the-meter applications in the United States, most DPV-plus-storage systems to date have been ACcoupled, in particular for retrofits and for larger commercial and industrial customers (Moskowitz 2017). When retrofitting existing PV systems under AC coupling, the battery system can be added in parallel without replacing the PV inverter. Retrofits under DC-coupling, however, require the PV inverter to be replaced, which can add significantly to project costs (Ardani et al. 2017). Commercial and industrial DPV-plus-storage systems are typically AC-coupled due to limited inverter sizing and system space constraints. Currently, the multiport hybrid inverters used in DC systems come in select sizes, and are usually better suited for smaller residential customers. Furthermore, DC-coupled systems require the batteries to be interspersed throughout the photovoltaic array, which can be an issue for larger systems if the customers have limited space (Moskowitz 2017). Despite these limitations, for smaller customers installing new DPV-plus-storage systems not limited by space or inverter sizing, DC-coupling offers several key advantages. Even though multiport hybrid inverters are more expensive than standard inverters, DC-coupling tends to have lower overall costs due to shared inverter components and reduced wiring costs. This cost reduction is particularly relevant for smaller systems, for which inverter equipment is a significant driver of total system costs. For systems in which the DPV generation is often stored and consumed at a later time, rather than instantaneously consumed at the time of generation, DC-coupled systems also have higher efficiencies than AC-coupled systems as they suffer fewer inefficient AC-to-DC and DC-to-AC conversions (Ardani et al. 2017). Furthermore, DC-coupled systems avoid PV clipping, in which some DPV generation is dumped due to power limitations of inverters, which tend to be undersized relative to the maximum PV array output (Moskowitz 2017). ${ }^{73}$ The combination of high efficiency and avoided clipping means DC-coupled systems may be better suited to maximize DPV generation to meet on-site demand and/or sell electricity back to the grid, improving system economics. Figure D- 1 and Figure D- 2 provide illustrative diagrams for AC-coupled and DC-coupled systems, respectively. Table D- 1 summarizes the differences between $\mathrm{AC}$ - and DC-coupled systems.

\footnotetext{
${ }^{71}$ Importantly, these illustrative diagrams are not equivalent to electrical line diagrams, but instead are intended to qualitatively describe the various storage-related design configurations.

${ }^{72}$ Stand-alone storage systems are not covered in this appendix. While storage-plus-DPV systems have been more common in the United States relative to stand-alone storage systems, stand-alone storage systems may be more prevalent in other country contexts depending on the frequency of load shedding events and the premium that customers place on reliable electricity services.

${ }^{73}$ For information on PV clipping, see https://pvpmc.sandia.gov/modeling-steps/dc-to-ac-conversion/inverter-saturation/ from Sandia National Laboratories’ PV Performance Modeling Collaborative.
} 


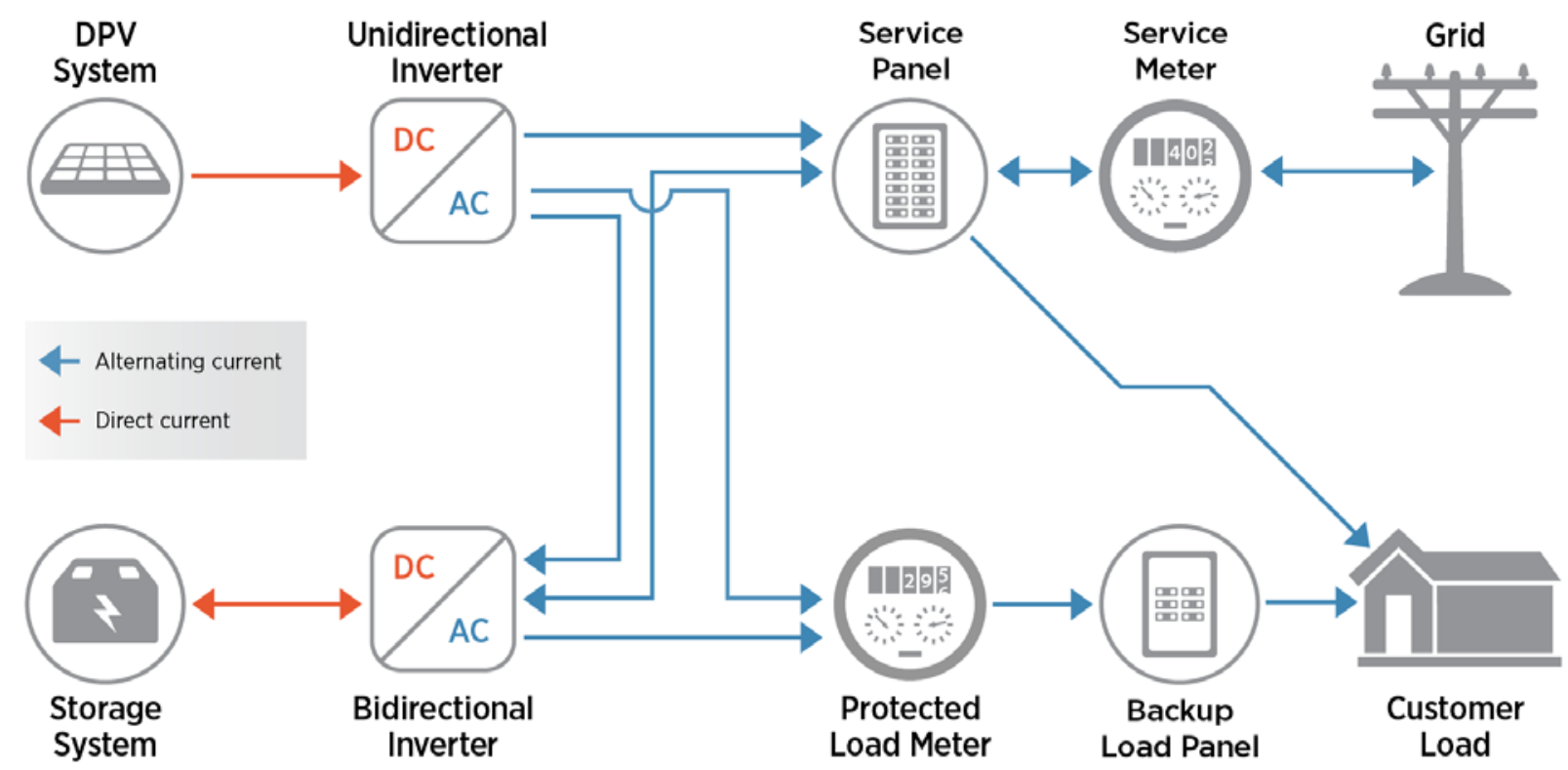

Figure D- 1. AC-coupled DPV-plus-storage system with backup enabled

Source: Original [IIlustration by Christopher Schwing, NREL]

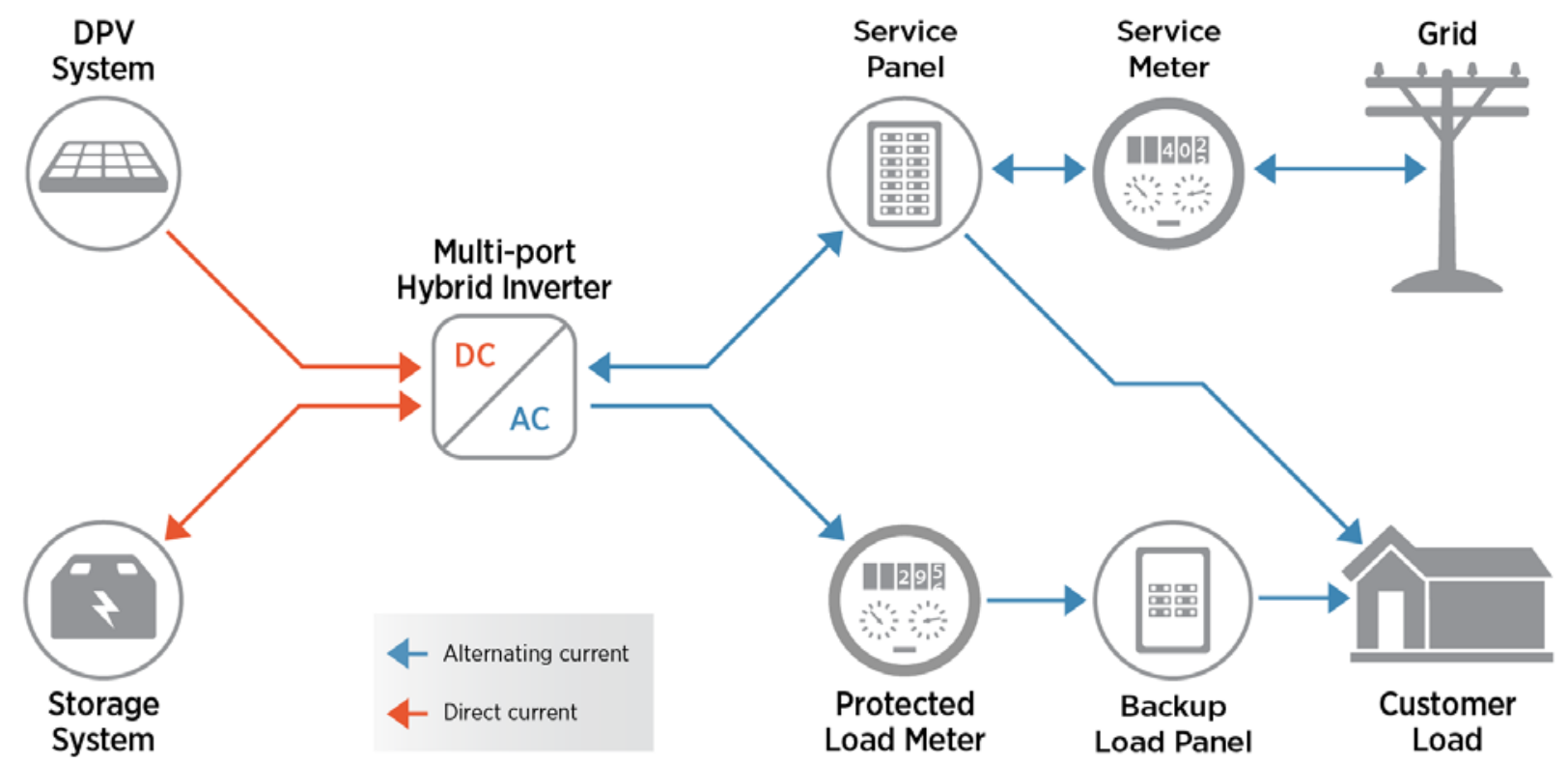

Figure D- 2. DC-coupled DPV-plus-storage system with multiport hybrid inverter with backup enabled

Source: Original [IIlustration by Christopher Schwing, NREL]

Regardless of how the DPV-plus-storage system is coupled, to continue supplying power during a grid outage, DPV-plus-storage systems require inverters capable of switching from the normal service entrance, which connects the customer to the DPV-plus-storage system and to the grid, to a special backup load panel. These backup load panels provide an entrance to the customer but not to the larger grid, allowing DPV-plus-storage to supply power to customers without endangering utility line workers. Configurations may also include a protected load meter, which only allows power to flow from the inverter to the backup power panel. Importantly, backup panels and protected load meters incur additional costs both for the equipment and installation. Furthermore, from 
an administrative standpoint, installers may need to work with customers to determine which customer loads should be served through the backup load panel in the event of a grid outage.

Table D- 1. Comparison of AC- and DC-Coupled Systems

\begin{tabular}{|c|c|c|}
\hline & DC-Coupled Systems & AC-Coupled Systems \\
\hline $\begin{array}{l}\text { Inverter } \\
\text { Requirements }\end{array}$ & $\begin{array}{l}\text { A single shared inverter, typically a multiport } \\
\text { hybrid inverter. }\end{array}$ & $\begin{array}{l}\text { Two separate inverters, one each for the } \\
\text { battery and PV array. }\end{array}$ \\
\hline Size Availability & $\begin{array}{l}\text { Multiport hybrid inverters come in a limited } \\
\text { number of sizes, normally at the residential } \\
\text { scale, although this may change as the } \\
\text { industry matures. }\end{array}$ & $\begin{array}{l}\text { A wide range of inverter sizes suitable for all } \\
\text { applications are available. }\end{array}$ \\
\hline $\begin{array}{l}\text { Space } \\
\text { Requirements }\end{array}$ & $\begin{array}{l}\text { DC-coupled systems tend to require more } \\
\text { space and must be co-located, as the } \\
\text { batteries are interspersed throughout the } \\
\text { array }\end{array}$ & $\begin{array}{l}\text { AC-coupled systems can have the battery and } \\
\text { PV array located completely independently of } \\
\text { one another. }\end{array}$ \\
\hline System Costs & $\begin{array}{l}\text { Tend to be less expensive for new projects } \\
\text { due to shared inverter components. This cost } \\
\text { reduction is particularly pronounced for small } \\
\text { systems, for which the inverter comprises a } \\
\text { larger share of total system costs. }\end{array}$ & $\begin{array}{l}\text { Tend to be more expensive for new projects } \\
\text { as inverters and associated equipment, wiring } \\
\text { and installation costs are duplicated. }\end{array}$ \\
\hline $\begin{array}{l}\text { System } \\
\text { Efficiency }\end{array}$ & $\begin{array}{l}\text { Tend to be more efficient in applications in } \\
\text { which the DPV generation is stored and } \\
\text { consumed at a later date. } \\
\text { Systems do not typically suffer from PV } \\
\text { clipping, increasing overall available PV } \\
\text { generation. }\end{array}$ & $\begin{array}{l}\text { Tend to be more efficient in applications in } \\
\text { which the PV generation is consumed } \\
\text { immediately. } \\
\text { PV generation is often clipped, due to power } \\
\text { constraints of the inverter, leading to lost } \\
\text { energy. }\end{array}$ \\
\hline $\begin{array}{l}\text { Suitability for } \\
\text { Retrofit Versus } \\
\text { New Build }\end{array}$ & $\begin{array}{l}\text { Better suited for new systems, as retrofitting } \\
\text { existing PV arrays with a battery requires } \\
\text { replacement of photovoltaic inverter. }\end{array}$ & $\begin{array}{l}\text { More common in retrofits as the battery can } \\
\text { be added to the system in parallel without } \\
\text { affecting the existing DPV system. }\end{array}$ \\
\hline $\begin{array}{l}\text { Appropriateness } \\
\text { for Customers }\end{array}$ & $\begin{array}{l}\text { Better suited for residential or smaller } \\
\text { commercial customers not subject to inverter } \\
\text { sizing limitations or space constraints. }\end{array}$ & $\begin{array}{l}\text { Better suited for larger commercial and } \\
\text { industrial customers or customers with siting } \\
\text { constraints. }\end{array}$ \\
\hline
\end{tabular}




\section{References}

Afonso, Swansy. 2020. "India's Electric Vehicle Ambitions Could Stumble on Lack of Lithium." News Blog. LiveMint. January 21, 2020. https://www.livemint.com/industry/energy/india-s-electricvehicle-ambitions-could-stumble-on-lack-of-lithium-11579576678742.html.

Akhil, Abbas, Georgianne Huff, Aileen Currier, Benjamin Kaun, Dan Rastler, Stella Bingqing Chen, Andrew Cotter, Dale Bradshaw, and William Gauntlett. 2013. "DOE/EPRI 2013 Electricity Storage Handbook in Collaboration with NRECA." Sandia National Laboratories. https://www.energy.gov/sites/prod/files/2013/08/f2/ElecStorageHndbk2013.pdf.

Ameren Illinois. 2019. "Power Smart Pricing." 2019. https://www.powersmartpricing.org/.

Anderson, Robert, and Jonathan O Burrows. 2017. "Supply Side DR Pilot 2015-2016 Summary and Findings (Public Version)." Olivine and PG\&E. http://olivineinc.com/wpcontent/uploads/2017/02/PDF_DemandResponseOIR-2013_Report_PGE_20171011-Public.pdf.

APS. 2018a. "Saver Choice Max.” Utility Tariff Rates. Service Plans. 2018. https://www.aps.com/en/residential/accountservices/serviceplans/Pages/saver-choice-max.aspx. 2018b. "Saver Choice Plus.” Utility Tariff Rates. Service Plans. 2018. https://www.aps.com/en/residential/accountservices/serviceplans/Pages/saver-choice-plus.aspx.

. 2018c. "APS Offers Three New Programs to Help Customers Use More Solar Energy.” News Brief. Latest News. September 2018. https://www.aps.com/en/ourcompany/news/latestnews/Pages/aps-offers-three-new-programs-tohelp-customers-use-more-solar-energy.aspx.

Ardani, Kristen, Eric O'Shaughnessy, Ran Fu, Chris McClurg, Joshua Huneycutt, and Robert Margolis. 2017. "Installed Cost Benchmarks and Deployment Barriers for Residential Solar Photovoltaics with Energy Storage: Q1 2016.” Technical Report NREL/TP-7A40-67474. Golden, CO: NREL. https://www.nrel.gov/docs/fy17osti/67474.pdf.

Baker, Charles D, Karyn E Polito, Kathleen A Theoharides, and Judith Judson. 2019. The Clean Peak Energy Standard. Draft Regulation Summary.

Baldassari, Mark. 2018. "Electronic Current Limit (ECL) Use Cases.” Enphase. https://mn.gov/puc/assets/ECL-UC-v2.4_tcm14-389728.pdf.

Bowen, Thomas, Ilya Chernyakhovskiy, and Paul Denholm. 2019. "Grid-Scale Battery Storage: Frequently Asked Questions.” Technical Report NREL/TP-6A20-74426. Golden, CO: NREL. https://www.nrel.gov/docs/fy19osti/74426.pdf.

Bridge to India. 2019. “India Solar Rooftop Map.” Gurugram, India: Bridge to India. https://bridgetoindia.com/report/india-solar-rooftop-map-december-2019/.

Buckley, Tim. 2015. "India's Electricity-Sector Transformation." Cleveland OH: Institute for Energy Economics and Financial Analysis. http://ieefa.org/wp-content/uploads/2015/08/IEEFA-IndianElectricity-Sector-Transformation-August-2015.pdf.

Bundesnetzagentur. 2019. "EEG Reg-is-ter Da-ta and Ref-er-ence Val-ues for Pay-ment.” Government Website. Renewable Energy. July 31, 2019. 
https://www.bundesnetzagentur.de/EN/Areas/Energy/Companies/RenewableEnergy/Facts_Figur es_EEG/Register_data_tariffs/EEG_registerdata_payments_node.html.

Burgess, Ed, and Todd Olinsky-Paul. 2018. "Energy Storage in the Clean Peak Standard." November 8. https://www.cesa.org/webinars/energy-storage-in-the-clean-peak-standard/.

Byrne, Ray. n.d. “Energy Storage Overview.” Accessed June 7, 2019. https://www.sandia.gov/essssl/docs/swpuc/Byrne_Energy_storage_101_SAND2016-4387.pdf.

California ISO. 2010. “Demand Response-Proxy Demand Resource.” August 10, 2010. http://www.caiso.com/informed/Pages/StakeholderProcesses/CompletedClosedStakeholderInitiat ives/DemandResponse-ProxyDemandResource.aspx.

- 2016. "Distributed Energy Resource Provider Participation Guide with Checklist." http://www.caiso.com/Documents/DistributedEnergyResourceProviderParticipationGuideandCh ecklist.pdf.

California State Legislature. 2017. "Bill Text - AB-546 Land Use: Local Ordinances: Energy Systems." 2017. https://leginfo.legislature.ca.gov/faces/billTextClient.xhtml?bill_id=201720180AB546.

CEA. 2018. “Load Generation Balance Report: 2018-2019.” New Delhi, India: Central Electricity Authority. http://cea.nic.in/reports/annual/lgbr/lgbr-2018.pdf.

—. 2019a. "Load Generation Balance Report: 2019-2020.” New Delhi, India: Central Electricity Authority. http://cea.nic.in/reports/annual/lgbr/lgbr-2019.pdf.

. 2019b. Ammendment to Technical Standards for Connectivity of the Distributed Generation Resources Regulation. Notification. Vol. No.12/X/STD(CONN)/GM/CEA/2018. http://www.cea.nic.in/reports/regulation/notified_080219.pdf.

CERC. 2018. "Re-Designing Real Time Electricity Market in India.” Discussion Paper No RA14026(11)/2/2018-CERC. New Delhi, India: Central Electricity Regulatory Commission (CERC). https://www.mahadiscom.in/consumer/wp-content/uploads/2018/08/Discussion-Paperon-Real-Time-Electricity-Market.pdf.

Chattopadhyay, Shashwata, Rajeev Dubey, Vivek Kuthanazhi, Sachin Zachariah, Sonali Bhaduri, Chiranjibi Mahapatra, Sugguna Rambabu, et al. 2017. All-India Survey of Photovoltaic Module Reliability: 2016. NCPRE, IITB and NISE, Gurugram.

https://www.researchgate.net/publication/322386040_AllIndia_Survey_of_Photovoltaic_Module_Reliability_2016.

ComEd. 2019. "Hourly Pricing Program.” 2019. https://hourlypricing.comed.com/.

Commonwealth of Massachusetts. 2018. Bill H.4857 An Act to Advance Clean Energy. https://malegislature.gov/Bills/190/H4857.

ConEdison. 2016. “Con Edison 'Virtual Power Plant' Program Combines Solar and Storage to Improve Grid Resiliency." June 13, 2016. https://www.coned.com/en/about-conedison/media/news/20160613/virtual-power-plant. 
Conover, DR. 2014. "Overview of Development and Deployment of Codes, Standards and Regulations Affecting Energy Storage System Safety in the United States." Technical Report PNNL-23578. PNNL. https://www.sandia.gov/ess-ssl/docs/safety/Codes_101_PNNL_23578.pdf.

Cook, Jeffrey J, Kristen Ardani, Eric O’Shaughnessy, Brittany Smith, and Robert Margolis. 2018. "Expanding PV Value: Lessons Learned from Utility-Led Distributed Energy Resource Aggregation in the United States." Technical Report NREL/TP-6A20-71984. https://www.nrel.gov/docs/fy19osti/71984.pdf.

CPUC. 2013. Order Instituting Rulemaking Pursuant to Assembly Bill 2514 to Consider the Adoption of Procurement Targets for Viable and Cost-Effective Energy Storage Systems. Decision 13-10040. http://docs.cpuc.ca.gov/PublishedDocs/Published/G000/M079/K533/79533378.pdf.

. 2014. Order Instituting Rulemaking Regarding Policies, Procedures and Rules for the California Solar Initiative, the Self-Generation Incentive Program and Other Distributed Generation Issues. Vol. Decision 14-05-033.

http://docs.cpuc.ca.gov/PublishedDocs/Published/G000/M091/K251/91251428.PDF.

. 2016a. "Decision Adopting Successor to Net Energy Metering Tariff." D. 16-01-044. San Francisco: CPUC. http://docs.cpuc.ca.gov/PublishedDocs/Published/G000/M158/K181/158181678.pdf.

. 2016b. "Order Instituting Rulemaking Regarding Policies, Procedures and Rules for California Solar Initiative, the Self-Generation Incentive Program and Other Distributed Generation Issues." Decision 16-04-020. http://docs.cpuc.ca.gov/PublishedDocs/Published/G000/M161/K480/161480050.PDF.

. 2017a. Decision Revising the Self-Generation Incentive Program Pusuant to Assembly Bill 1637 and Granting the Petition for Modification of Decision 16-06-055 by the California Solar Energy Industry Association. Vol. Decision 17-04-17.

http://docs.cpuc.ca.gov/PublishedDocs/Published/G000/M183/K843/183843620.PDF.

. 2017b. Order Instituting Rulemaking to Consider Streamlining Interconnection of Distributed Energy Resources and Improvements to Rule 21. Vol. Rulemaking 17-07-007. http://docs.cpuc.ca.gov/PublishedDocs/Published/G000/M192/K079/192079467.docx.

2018a. Decision On Multiple-Use Application Issues. Vol. Decision 18-01-003. http://docs.cpuc.ca.gov/PublishedDocs/Published/G000/M206/K462/206462341.PDF.

. 2018b. Order Instituting Rulemaking to Develop a Successor to Existing Net Energy Metering Tariffs Pursuant to Public Utilities Code Section 2827.1, and to Address Other Issues Related to Net Energy Metering. Vol. Decision 18-02-008. http://docs.cpuc.ca.gov/PublishedDocs/Published/G000/M210/K927/210927448.PDF.

. 2019a. "Self-Generation Incentive Program.” Agency Website. Consumer Energy Resources. 2019. https://www.cpuc.ca.gov/sgip/.

- 2019b. "About the Self-Generation Incentive Program.” Agency Website. Consumer Energy Resources. 2019. https://www.cpuc.ca.gov/General.aspx?id=11430.

Customized Energy Solutions, Alevo Analytics, Sustainable Energy Advantage LLC, and Daymark Energy Advisors. 2017. "State of Charge: Massachusetts Energy Storage Initiative Study." 
Energy Storage Initiative. Boston, MA: Department of Energy Resources. https://www.mass.gov/files/2017-07/state-of-charge-report.pdf\#page=192.

Darghouth, Naïm R., Galen L. Barbose, and Andrew D. Mills. 2019. "Implications of Rate Design for the Customer-Economics of Behind-the-Meter Storage.” Berkeley, CA: LBNL. https://emp.lbl.gov/publications/implications-rate-design-customer.

Darghouth, Naïm R., Galen L. Barbose, Andrew D. Mills, Ryan H. Wiser, Pieter Gagnon, and Lori Bird. 2017. "Exploring Demand Charge Savings from Commercial Solar." Berkeley, CA: LBNL and NREL. https://emp.lbl.gov/publications/exploring-demand-charge-savings- 0 .

Denholm, Paul, Matthew O’Connell, Gregory Brinkman, and Jennie Jorgenson. 2015. “Overgeneration from Solar Energy in California: A Field Guide to the Duck Chart." Technical Report NREL/TP6A20-65023. Golden, CO: NREL. https://www.nrel.gov/docs/fy16osti/65023.pdf.

Denholm, Paul. 2019. "Greening the Grid: Utility-Scale Battery Storage.” February. https://cleanenergysolutions.org/training/greening-grid-utility-scale-battery-storage.

Department of Energy Resources. 2017. Solar Massachusetts Renewable Target (SMART) Program. https://www.mass.gov/files/documents/2017/10/16/225cmr20.pdf.

Diau, Kevin. 2017. “How California's New Time-of-Use Rates Will Affect C\&I Customers Considering Solar PV." Greentech Media. December 11, 2017.

https://www.greentechmedia.com/articles/read/california-time-of-use-commercial-customerssolar-pv.

Ding, Fei, Barry Mather, and Peter Gotseff. 2016. “Technologies to Increase PV Hosting Capacity in Distribution Feeders: Preprint.” In , 7. Boston, MA. https://www.nrel.gov/docs/fy16osti/65995.pdf.

DOER. 2017. "Guideline on Energy Storage." https://www.mass.gov/files/documents/2018/07/13/Energy\%20Storage\%20Guideline\%20DRAF T\%20071318.pdf.

. 2018. "Solar Massachusetts Renewable Target Program: Guideline on Energy Storage." Boston, MA: Department of Energy Resources. https://www.mass.gov/files/documents/2018/09/13/Energy\%20Storage\%20Guideline\%20FINAL \%20091318.pdf.

Donalds, Samantha. 2017. "Distributed Generation in State Renewable Portfolio Standards." The RPS Collaborative. Montpelier, VT: Clean Energy States Alliance. https://www.cesa.org/assets/2017Files/DG-RPS.pdf.

Fan, Xiayue, Bin Liu, Jie Liu, Jia Ding, Xiaopeng Han, Yida Deng, Xiaojun Lv, et al. 2020. "Battery Technologies for Grid-Level Large-Scale Electrical Energy Storage.” Transactions of Tianjin University, January. https://doi.org/10.1007/s12209-019-00231-w.

Faruqui, Ahmad. 2018. "Modernizing Distribution Tariffs for Households." presented at the Energy Consumers Association, Sydney, Australia, November.

https://brattlefiles.blob.core.windows.net/files/14848_modernizing_distribution_tariffs_for_hous eholds_11-07-2018.pdf. 
Faruqui, Ahmad, Ryan Hledik, and Jennifer Palmer. 2012. "Time-Varying and Dynamic Rate Design." Global Power Best Practice Series. Regulatory Assistance Project.

https://www.raponline.org/wp-content/uploads/2016/05/rap-faruquihledikpalmertimevaryingdynamicratedesign-2012-jul-23.pdf.

FERC. 2018a. "Distributed Energy Resources Technical - Considerations for the Bulk Power System." Staff Report Docket No. AD18-10-000. FERC. https://www.ferc.gov/legal/staffreports/2018/der-report.pdf.

. 2018b. "Electric Storage Participation in Markets Operated by Regional Transmission Organizations and Independent System Operators." Order. FERC. https://www.ferc.gov/whatsnew/comm-meet/2018/021518/E-1.pdf.

FOR. 2019. "Draft Model Regulation for Grid Interactive Distributed Renewable Energy Sources." http://www.forumofregulators.gov.in/Data/Reports/DMR-for-GIDRES-08-05-19.pdf.

2018. "Report on Power Quality of Electricity Supply to the Consumers.” New Delhi, India: Forum of Regulators. http://www.forumofregulators.gov.in/Data/Reports/Power07.pdf.

GERC. 2016. Regulations for Net Metering Rooftop Solar PV Grid Interactive Systems. Notification No. $5 / 2016$. http://www.cbip.org/Regulations2018/Data/Gujarat/GJ_2/2_GJ_Net_Metering_Consolidate.pdf.

GOI. 2018. "Report of the High Level Empowered Committee to Address the Issues of Stressed Thermal Power Projects.” New Delhi, India: Government of India - High Level Empowered Committee. https://powermin.nic.in/sites/default/files/webform/notices/20_Nov_R1_Draft_Report_HLEC_Fi nal_20_Nov.pdf.

—. 2019a. "Ujwal DISCOM Assurance Yojana.” Home Page. Ministry of Power - Government of India. 2019. https://www.uday.gov.in/home.php.

. 2019b. "Mobility Solutions Get a Boost." Press Release. National Mission on Transformative Mobility and Battery Storage. March 7, 2019. pib.gov.in/Pressreleaseshare.aspx?PRID=1567807.

Golden, Thomas A., Patricia A. Haddad, Bradley H. Jones, Marc R. Pacheco, and Patrick M. O’Connor. 2018. An Act to Advance Clean Energy. https://malegislature.gov/Bills/190/H4857.

Governor's Office of Planning and Research. 2019. "Renewable Energy - Office of Planning and Research.” 2019. http://opr.ca.gov/planning/land-use/renewable-energy/.

GTM, and Energy Storage Association. 2019. "U.S. Energy Storage Monitor: Q2 2019 Full Report.” https://www.woodmac.com/research/products/power-and-renewables/us-energy-storagemonitor/.

Gupta, Mitalee. 2019. "Solar-plus-Storage Sytsem Architectures: Design, Pricing, and Economics in the U.S.” Wood Mackenzie.

Hawaii PUC. 2018. Instituting a Proceeding to Investigate Distributed Energy Resource Policies. 33258 . https://dms.puc.hawaii.gov/dms/DocumentViewer?pid=A1001001A18G02B00134C00952. 
HECO. 2015a. Rule 14 Service Connections and Facilities on Customer's Premises.

https://www.hawaiianelectric.com/Documents/my_account/rates/hawaiian_electric_rules/14.pdf.

. 2015b. "Hawaiian Electric and Stem, Inc. Bring Intelligent Energy Storage to Oahu." Press

Release. Hawaiian Electric. October 2015. https://www.hawaiianelectric.com/hawaiian-electricand-stem-inc-bring-intelligent-energy-storage-to-oahu.

2017. "Hawaiian Electric and Stem, Inc. Successfully Test 1 MW of Energy Storage at 29 Commercial Customer Sites.” Press Release. Hawaiian Electric. January 2017. https://www.hawaiianelectric.com/hawaiian-electric-and-stem-inc-successfully-test-1-mw-ofenergy-storage-at-29-commercial-customer-sites.

. 2018. Rule No. 25 Smart Export Program.

https://www.hawaiianelectric.com/documents/billing_and_payment/rates/hawaiian_electric_rule s/25.pdf.

.2019a. “Customer Self-Supply.” Utility Tariff Rates. Customer Renewable Programs. 2019. https://www.hawaiianelectric.com/products-and-services/customer-renewableprograms/customer-self-supply.

. 2019b. “Smart Export.” Utility Tariff Rates. Customer Renewable Programs. 2019.

https://www.hawaiianelectric.com/products-and-services/customer-renewable-programs/smartexport.

HECO. 2020. "Net Energy Metering." Utility Tariff Rates. Customer Renewable Programs. 2020. http://www.hawaiianelectric.com/products-and-services/customer-renewable-programs/privaterooftop-solar/net-energy-metering.

Hledik, Ryan, and Gus Greenstein. 2016. "The Distributional Impacts of Residential Demand Charges." The Electricity Journal 29 (6): 33-41. https://doi.org/10.1016/j.tej.2016.07.002.

Hogan, William. 2014. "Time-of-Use Rates and Real-Time Prices.” Harvard. https://sites.hks.harvard.edu/fs/whogan/Hogan_TOU_RTP_Newark_082314.pdf.

Holt, Edward A., and Todd Olinksy-Paul. 2016. "Does Energy Storage Fit in an RPS?" Clean Energy States Alliance. https://www.cesa.org/assets/2016-Files/Energy-Storage-and-RPS-Holt.pdf.

Horowitz, Kelsey, Zachary Peterson, Michael Coddington, Fei Ding, Ben Sigrin, Danish Saleem, Sarah E Baldwin, et al. 2019. "An Overview of Distributed Energy Resource (DER) Interconnection: Current Practices and Emerging Solutions.” Technical Report NREL/TP-6A20-72102. Golden, CO: NREL. https://www.nrel.gov/docs/fy19osti/72102.pdf.

Hyde, Tripp. 2018. "Challenges for Batteries in the California Fire Code." PV Magazine, August 7, 2018. https://pv-magazine-usa.com/2018/08/07/challenges-for-batteries-in-the-california-firecode/.

IEA. 2018. "Status of Power System Transformation.” Paris, France: International Energy Agency. https://webstore.iea.org/status-of-power-system-transformation-2018.

- 2019a. "Policies Database." 2019. https://www.iea.org/policies. 
—. 2019b. "Renewables 2018 - Analysis and Forecasts to 2023." Market Report Series.

International Energy Agency. https://www.iea.org/renewables2018/.

IFC. 2019. "The Dirty Footprint of the Broken Grid: The Impacts of Fossil Fuel Back-up Generators in Developing Countries.” Washington, DC: International Finance Corporation.

https://www.ifc.org/wps/wcm/connect/industry_ext_content/ifc_external_corporate_site/financia 1+institutions/resources/dirty-footprint-of-broken-grid.

India Smart Grid Forum. 2019. "Energy Storage System - Roadmap for India: 2019-2032.” India Smart Grid Forum. https://niti.gov.in/sites/default/files/2019-11/ISGF.pdf.

Jaiswal, Shantanu, Atin Jain, Itamar Orlandi, and Ashish Sethia. 2017. “Accelerating India’s Clean Energy Transition." Bloomberg New Energy Finance. https://data.bloomberglp.com/professional/sites/10/BNEF_Accelerating-Indias-Clean-EnergyTransition_Nov-2017.pdf.

Jimenez, Lupe. 2014. "Residential Energy Smart Community Demonstration.” Sacramento, April 28. http://smartgridcustomereducation.com/presentations/SGCES-LupeJimenez-SMUD.pdf.

Josey, Ann, Shantanu Dixit, Ashwini Chitnis, and Ashwin Gambhir. 2018. "Electricity Distribution Companies in India: Preparing for an Uncertain Future.” Pune, India: Prayas Energy Group. http://www.prayaspune.org/peg/publications/item/377.

KERC. 2019. Decision on Various Models and Guidelines for Solar Rooftop Photovoltaic Plants Allowed to Be Installed on Rooftops of the Consumers' Buildings. https://karunadu.karnataka.gov.in/kerc/Documents/Decision\%20on\%20various\%20models\%20a nd\%20Guidelines\%20for\%20SRTPV\%20allowed\%20to\%20be\%20installed\%20on\%20rooftops $\% 20$ of $\% 20$ consumer\%20buildings.pdf.

KPMG. 2015. "Taxes and Incentives for Renewable Energy." Amstelveen, the Netherlands: KPMG. https://assets.kpmg/content/dam/kpmg/pdf/2015/09/taxes-and-incentives-2015-web-v2.pdf.

Kuldeep, Neeraj, Kumaresh Ramesh, Akanksha Tyagi, and Selna Saji. 2019. "Valuing Grid-Conencted Rooftop Solar: A Framework to Assess Costs and Benefits to Discoms." New Delhi, India: Council on Energy, Environment and Water. https://www.ceew.in/sites/default/files/Valuingreport.pdf.

Lazar, Jim. 2014. "Electric Utility Residential Customer Charges and Minimum Bills: Alternative Approaches for Recovering Basic Distribution Costs.” Montpelier, VT: Regulatory Assistance Project. https://www.raponline.org/wp-content/uploads/2016/05/rap-lazarelectricutilityresidentialcustomerchargesminimumbills-2014-nov.pdf.

Lazar, Jim, and Wilson Gonzalez. 2015. "Smart Rate Design for a Smart Future.” Montpelier, VT: Regulatory Assistance Project. http://www.raponline.org/document/download/id/7680.

Lazar, Jim, Frederick Weston, Wayne Shirley, Janine Migden-Ostrander, Dave Lamont, and Elizabeth Watson. 2016. "Revenue Regulation and Decoupling: A Guide to Theory and Application." Montpelier, VT: Regulatory Assistance Project. https:/www.raponline.org/knowledgecenter/revenue-regulation-and-decoupling-a-guide-to-theory-and-application-incl-case-studies/.

Linvill, Carl, John Shenot, and Jim Lazar. 2013. "Designing Distributed Generation Tariffs Well: Fair Compensation in a Time of Transition.” Montpelier, VT: Regulatory Assistance Project. 
http://www.raponline.org/wp-content/uploads/2016/05/rap-linvillshenotlazar-faircompensation2013-nov-27.pdf.

Littell, David, Camille Kadoch, Phil Baker, Ranjit Bharvirkar, Max Dupuy, Brenda Hausauer, Carl Linvill, et al. 2017. "Next-Generation Performance-Based Regulation: Emphasizing Utility Performance to Unleash Power Sector Innovation." Technical Report NREL/TP-6A50-68512. 21CPP and RAP. https://www.nrel.gov/docs/fy17osti/68512.pdf.

Lowder, Travis, Ella Zhou, and Tian Tian. 2017. "Evolving Distributed Generation Support Mechanisms: Case Studies from United States, Germany, United Kingdom, and Australia.” NREL/TP--6A20-67613, 1347278. https://doi.org/10.2172/1347278.

McAllister, Richard, David Manning, Lori Bird, Michael Coddington, and Christina Volpi. 2019. "New Approaches to Distributed PV Interconnection: Implementation Considerations for Addressing Emerging Issues." Technical Report NREL/TP-6A20-72038. Golden, CO: National Renewable Energy Laboratory. https://www.nrel.gov/docs/fy19osti/72038.pdf.

McLaren, Joyce, Nick Laws, Kate Anderson, Nick DiOrio, and Hannah Miller. 2019. “Solar-plusStorage Economics: What Works Where, and Why?" The Electricity Journal 32 (1): 28-46. https://doi.org/10.1016/j.tej.2019.01.006.

Ministry of Power. 2019. "Payment Ratification and Analysis in Power Procurement for Bringing Transparency in Invoicing of Generators." Government Dataset. PRAAPTI. November 2019. https://www.praapti.in/.

MIT Electric Vehicle Team. 2008. “A Guide to Understanding Battery Specifications.” MIT. http://web.mit.edu/evt/summary_battery_specifications.pdf.

MNRE. 2014. Off-Grid and Decentralized Solar Application Scheme: Operational Guidelines for Grid Connected Rooftop and Small Solar Power Plants Programme. Annexure. Vol. 30/11/201213/NSM. https://mnre.gov.in/file-manager/UserFiles/Scheme-Grid-Connected-Rooftop-\&-smallsolar-power-plants.pdf.

. 2015. State Wise and Year Wise Targets for Installation of 40,000 MW Grid Connected Solar Rooftop Systems. Vol. D.O. No. 03/13/2015-16/GCRT.

https://mnre.gov.in/sites/default/files/webform/notices/State-wise-and-year-wise-target-forinstallation-of-40000MWp-GCRT-systems_0.pdf.

. 2018. Long Term Growth Trajectory of Renewable Purchase Obligations (RPOs) for Solar and Non-Solar for a Period of Three Years. Vol. No.23/03/2016-R\&R.

https://powermin.nic.in/sites/default/files/webform/notices/RPO_trajectory_2019-

22_Order_dated_14_June_2018.pdf.

. 2019. Guidelines on Implementation of Phase-II of Grid Connected Rooftop Solar Programme for Achieving 40 GW Capacity from Rooftop Solar by the Year 2022. Office Memorandum. Vol. No. 318/331/2017. https://mnre.gov.in/sites/default/files/schemes/Notification-20082019184419.pdf.

Morris, Jeff, Cindy Ryu, and Marko Liias. 2013. Regarding the Use of an Energy Storage Facility to Meet Annual Targets under RCW 19.285.040.

https://apps.leg.wa.gov/billsummary/?BillNumber=1289\&Year=2013\&Initiative=false\#history. 
Moskowitz, Scott. 2017. "The Energy Storage Inverter Landscape: Key Trends in an Emerging Market." GTM Research. https://www.woodmac.com/our-expertise/focus/Power--Renewables/TheEnergy-Storage-Inverter-Landscape/.

Mullendore, Seth, and Lewis Milford. 2015. "Solar+Storage 101: An Introductory Guide to Resilient Power Systems.” Clean Energy Group. https://www.cleanegroup.org/wpcontent/uploads/Energy-Storage-101.pdf.

Nagarajan, Adarsh. 2018. "Best Practices for Grid Codes for Renewable Energy Generators." Webinar presented at the Greening the Grid, October. https://cleanenergysolutions.org/training/gridcodes-best-practices.

Neubauer, J, and M Simpson. 2015. "Deployment of Behind-The-Meter Energy Storage for Demand Charge Reduction.” Technical Report NREL/TP-5400-63162. NREL. https://www.nrel.gov/docs/fy15osti/63162.pdf.

Noh, Jin, Jamie Dickerson, and Todd Olinsky-Paul. 2018. "Solar + Storage in Net Metering Programs." https://www.cesa.org/assets/2018-Files/ES-webinar-slides-9-13-2018.pdf.

NV Energy. 2018a. Generating Facility Interconnections. Tariff No. 1-B, Rule No. 15. https://www.nvenergy.com/publish/content/dam/nvenergy/brochures_arch/about-nvenergy/ratesregulatory/electric-rules-south/Rule_15_South.pdf.

. 2018b. "Solar Incentives \& Energy Storage Incentives: Programs Handbook." Las Vegas, NV: NV Energy.

https://www.nvenergy.com/publish/content/dam/nvenergy/brochures_arch/cleanenergy/handboo ks/SolarGenerations-Handbook.pdf.

NYISO. 2017. "The State of Storage.” NYISO.

https://www.nyiso.com/documents/20142/3067443/State_of_Storage_Report_Final_1Dec2017.p df/188a42d7-5191-a0e1-52e6-84d15868f3df.

NYPSC. 2015. Order Adopting Regulatory Policy Framework and Implementation Plan. Proceeding on Motion of the Commission in Regard to Reforming the Energy Vision. http://documents.dps.ny.gov/public/Common/ViewDoc.aspx?DocRefId=\%7B0B599D87-445B4197-9815-24C27623A6A0\%7D.

. 2017. "Standardized Interconnection Requirements and Application Process for New Distributed Generators and Energy Storage Systems 5 MW or Less Connected in Parallel with Utility Distribution Systems.” New York, NY: New York Public Service Commission. http://documents.dps.ny.gov/public/Common/ViewDoc.aspx?DocRefId=\%7BA7780F50-4D4D45D4-8B83-A1832488C12D\%7D.

2018a. Order Establishing Energy Storage Goal and Deployment. Vol. Case 18-E-0130. http://documents.dps.ny.gov/public/Common/ViewDoc.aspx?DocRefId=\{FDE2C318-277F4701-B7D6-C70FCE0C6266\} .

. 2018b. Order Implementing Hybrid Energy Storage System Tariff. Vol. Case 15-E-0751. http://documents.dps.ny.gov/public/Common/ViewDoc.aspx?DocRefId=\%7BB3CCA2B724A0-47E1-88B2-5FE0ED521720\%7D. 
NYSEG. 2018. “Aggregated Behind the Meter Energy Storage.” Implementation Plan. New York Enery Storage Initiative. Binghamton, NY: New York State Electric and Gas Corporation. http://documents.dps.ny.gov/public/Common/ViewDoc.aspx?DocRefId=\%7B21C88AB8-D42E4455-A3E7-42046E7FC547\%7D.

Parikh, Anjana. 2020. “Concerns Over Maharashtra's Plan to Impose Grid Support Charges on Rooftop Solar.” Mercom India (blog). February 18, 2020. https://mercomindia.com/concernsmaharashtra-impose-grid-support-charges-rooftop-solar/.Passey, Robert, Navid Haghdadi, Anna Bruce, and Iain MacGill. 2017. "Designing More Cost Reflective Electricity Network Tariffs with Demand Charges." Energy Policy 109 (October): 642-49.

https://doi.org/10.1016/j.enpol.2017.07.045.

Patel, Dheer, Joni Sliger, and Ranjit Bharvirkar. (forthcoming). "Demand Flexibility and Distributed Energy Resources: Examining Four Options for India's Power Distribution Sector to Become Cleaner, More Affordable, and More Reliable.” Montpelier, VT: Regulatory Assistance Project.

Peterson, Zachary. 2018. "Emerging Practices for Energy Storage Interconnection." Emerging Practices for Energy Storage Interconnection (blog). 2018. https:/www.nrel.gov/dgic/interconnectioninsights-2018-11.html.

Peterson, Zachary, and Emerson Reiter. 2017. "DGIC Interconnection Insights.” Blog. Distributed Generation Integration Collaborative. October 2017. https://www.nrel.gov/dgic/interconnectioninsights-2017-10.html.

PG\&E. 2018. Electric Rule No. 21: Generating Facility Interconnections. https://www.pge.com/tariffs/tm2/pdf/ELEC_RULES_21.pdf.

PG\&E, and Olivine. 2017. "PG\&E 2016 XSP and SSP/SSP II Update.” March. http://olivineinc.com/wp-content/uploads/2017/02/pge_pilots_update_for_ed-20170322.pdf.

Prayas Energy Group. 2019. "Electricity Supply Monitoring Initiative: Watch Your Power Quality." Summary Analysis. Pune, India: Prayas Energy Group. http://watchyourpower.org/uploaded_reports.php. . 2019. "Electricity Supply: Quality Issues Persist.” Blog. Energy Consumption of Households (ECHO) (blog). 2019. http://www.prayaspune.org/peg/electricity-supply-quality-issuespersist.Proudlove, Autumn, Brian Lips, and David Sarkisian. 2018. "50 States of Solar, Q3 2018 Quarterly Report - Executive Summary." NC Clean Energy Technology Center. https://nccleantech.ncsu.edu/wp-content/uploads/2018/10/Q3-18_SolarExecSummary_Final.pdf.

PUA. 2014. “The Israeli Net Metering Scheme - Lessons Learned.” Israel Public Utility Authority for Electricity. https://pua.gov.il/English/Documents/The\%20Israeli\%20Net\%20Metering\%20Scheme\%20\%201 essons\%20learned.pdf.

PUCN. 2017. Rule 15 - Generating Facility Interconnections.

RBI. 2017. "State Finances: A Study of Budgets of 2016-2017.” Mumbai: Reserve Bank of India. https://rbidocs.rbi.org.in/rdocs/Publications/PDFs/0SF2016_12051728F3E926CFFB4520A027A C753ACF469A.PDF. 
REV Connect. 2018. “Lessons from REV Demos in New York’s Energy System.” January. https://nyrevconnect.com/wp-content/uploads/2018/01/Webinar2_Demo-Principles-v6-01-1718.pdf.

Rickerson, Wilson, Toby Couture, Galen L. Barbose, David Jacobs, Giles Parkinson, Emily Chessin, Andy Belden, Holly Wilson, and Henry Barrett. 2014. "Residential Prosumers: Drivers and Policy Options (Re-Prosumers).” International Energy Agency (IEA)- Renewable Energy Technology Deployment. http://iea-retd.org/wp-content/uploads/2014/09/REPROSUMERS_IEA-RETD_2014.pdf.

SCE. 2019. “Time-Of-Use Residential Rate Plans.” Utility Tariff Rates. Rates. 2019. http://www.sce.com/residential/rates/Time-Of-Use-Residential-Rate-Plans.

SCE, PG\&E, SDG\&E, SoCalGas, and SempraEnergy. 2017. "Self-Generation Incentive Program." Handbook. https://www.selfgenca.com/documents/handbook/2017.

Seetharam, Deva P., Ankit Agrawal, Tanuja Ganu, Jagabondhu Hazra, Venkat Rajaraman, and Rajesh Kunnath. 2013. "Hidden Costs of Power Cuts and Battery Backups." In Proceedings of the Fourth International Conference on Future Energy Systems, 39-50. E-Energy '13. Berkeley, California, USA: ACM, New York, NY. https://doi.org/10.1145/2487166.2487171.

Sharma, Yogima. 2020. "Niti Aayog Seeks Cabinet Nod for Battery Push." News. The Economic Times. January 24, 2020. https://economictimes.indiatimes.com/news/economy/policy/niti-aayog-seekscabinet-nod-for-battery-push/articleshow/73569360.cms.

Skinner. 2010. An Act to Amend Section 9620 of, and to Add Chapter 7.7 (Commencing with Section 2835) to Part 2 of Division 1 of, the Public Utilities Code, Relating to Energy. http://leginfo.legislature.ca.gov/faces/billTextClient.xhtml?bill_id=200920100AB2514.

Sovereign Energy, and Clean Coalition. 2017. "Task 2.10 : Final Report on Policy Recommendations and Guidelines for Permitting Energy Storage." http:/www.clean-coalition.org/site/wpcontent/uploads/2018/01/PAEC-Task-2.10_Final-Report-on-Policy-RecommendationsGuidelines-for-Permitting-Energy-Storage-03_wb-19-Jun-2017.pdf.

Spector, Julian. 2019. "Massachusetts Storage Market Imperiled by Requirement to Buy New Utility Meters.” News Blog. Greentech Media. July 25, 2019. https://www.greentechmedia.com/articles/read/massachusetts-solar-market-imperiled-byrequirement-to-pay-for-new-utility-.

SRP. 2018. "Reserve Your Battery Storage Incentive.” Battery Storage. May 1, 2018. https://srpnet.com/electric/home/batterystorage/default.aspx.

Texas Power Guide. 2017. "Indexed and Time-of-Use Plans Roundup.” Blog. Texas Power Guide (blog). May 11, 2017. https://www.texaspowerguide.com/2017/indexed-tou-plans/.

Tobin, Andy. 2018. “Arizona Energy Modernization Plan (AZEMP) and Clean Resource Energy Standard \& Tariff (CREST) Rulemaking Docket (RU-00000A-18-028," October 4, 2018. http://docket.images.azcc.gov/0000192672.pdf.

Tobin, Andy, and Nicholas Loper. n.d. “Arizona Energy Modernization Plan,” 13. 
Tokash, William, and Anissa Dehamma. 2017. "Distributed Solar PV Plus Energy Storage Systems Solar PV and Advanced Battery Energy Storage Applications for Residential, Commercial and Industrial, and Remote, Off-Grid Sectors." Global Market Analysis and Forecasts. Navigant. http://splunk01.nrel.gov/store/Navigant/Navigant2017/Navigant_DSPPESS_Q12017.pdf.

Trabish, Herman. 2015. "From Theory to Practice: The Challenges in Moving to 'Utility 2.0." News Blog. Utility Dive. October 27, 2015. https://www.utilitydive.com/news/from-theory-to-practicethe-challenges-in-moving-to-utility-20/407919/.

Ukar, Asier. 2017. "Pilot Study on Quality Aspects of PV Power Plants in India." Report Summary. Berlin, Germany: PI Photovoltaik Institut. https://www.piberlin.com/images/pdf/publication/White_Paper_-_India_PV_Projects_Study_2017PI_Berlin.pdf.

Walton, Robert. 2018. "Massachusetts Approves New Demand Charge for Eversource's Net Metering Customers.” News Blog. Utility Dive. January 10, 2018. https://www.utilitydive.com/news/massachusetts-approves-new-demand-charge-for-eversourcesnet-metering-cust $/ 514477 /$.

Whited, Melissa, Tim Woolf, and Alice Napoleon. 2015. "Utility Performance Incentive Mechanisms: A Handbook for Regulators.” Cambridge, MA: Synapse Energy Economics. https://www.synapseenergy.com/sites/default/files/Utility\%20Performance\%20Incentive\%20Mechanisms\%2014098_0.pdf.

Xcel Energy. 2017. Guidance No. 1 for the Interconnection of Electric Storage as Stand-Alone Sources, Parallel Operation for Customers without Generation, and in Parallel with Self-Generation. https://www.xcelenergy.com/staticfiles/xeresponsive/Programs\%20and\%20Rebates/Residential/CO-solar-residence-Storage-Guidance1.pdf.

2018. Electric Rate Book: Colorado Rates and Regulations. https://www.xcelenergy.com/staticfiles/xe/PDF/Regulatory/CO-Rates-\&-Regulations-EntireElectric-Book.pdf.

Zinaman, Owen R, Alexandra Aznar, Carl Linvill, Naim Darghouth, Emanuele Bianco, and Timon Dubbeling. 2017. "Grid-Connected Distributed Generation: Compensation Mechanism Basics." Brochure NREL/BR-6A20-68469. NREL. https://www.nrel.gov/docs/fy18osti/68469.pdf. 


\section{www.greeningthegrid.org | www.nrel.gov/usaid-partnership}

Jeremy Foster

U.S. Agency for International Development Email: jfoster@usaid.gov

Sarah Lawson

U.S. Agency for International Development Email: slawson@usaid.gov

Ilya Chernyakhovskiy

National Renewable Energy Laboratory

Email: ilya.chernyakhovskiy@nrel.gov
Greening the Grid is a platform for expertly curated information, tools, and technical assistance to support countries in power system transformation and grid modernization. Greening the Grid is supported by the U.S. Agency for International Development.

The USAID-NREL Partnership addresses critical challenges to scaling up advanced energy systems through global tools and technical assistance, including the Renewable Energy Data Explorer, Greening the Grid, the International Jobs and Economic Development Impacts tool, and the Resilient Energy Platform. More information can be found at: www.nrel.gov/usaid-partnership

\section{( USAID RNREL}

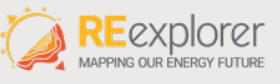

Greening the Grid

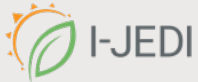

Resilient Energy 